San Jose State University

SJSU ScholarWorks

Master's Theses

Master's Theses and Graduate Research

Fall 2017

\title{
A Full Factorial Analysis of Polymerization Conditions of Sodium Alginate Beads to Determine the Effect on Diffusivity and Concentration Limit of Tryptophan and Glucose
}

Allison Schulkins

San Jose State University

Follow this and additional works at: https://scholarworks.sjsu.edu/etd_theses

\section{Recommended Citation}

Schulkins, Allison, "A Full Factorial Analysis of Polymerization Conditions of Sodium Alginate Beads to Determine the Effect on Diffusivity and Concentration Limit of Tryptophan and Glucose" (2017). Master's Theses. 4884.

DOI: https://doi.org/10.31979/etd.p847-a8jc

https://scholarworks.sjsu.edu/etd_theses/4884

This Thesis is brought to you for free and open access by the Master's Theses and Graduate Research at SJSU ScholarWorks. It has been accepted for inclusion in Master's Theses by an authorized administrator of SJSU ScholarWorks. For more information, please contact scholarworks@sjsu.edu. 
A FULL FACTORIAL ANALYSIS OF POLYMERIZATION CONDITIONS OF SODIUM ALGINATE BEADS TO DETERMINE THE EFFECT ON DIFFUSIVITY AND CONCENTRATION LIMIT OF TRYPTOPHAN AND GLUCOSE

\author{
A Thesis \\ Presented to \\ The Faculty of the Department of Biomedical, Chemical, and Materials Engineering \\ San José State University \\ In Partial Fulfillment \\ of the Requirements for the Degree \\ Master of Science
}

by

Allison Schulkins

December 2017 
(C) 2017

Allison Schulkins

ALL RIGHTS RESERVED 
The Designated Thesis Committee Approves the Thesis Titled

A FULL FACTORIAL ANALYSIS OF POLYMERIZATION CONDITIONS OF SODIUM ALGINATE BEADS TO DETERMINE THE EFFECT ON DIFFUSIVITY AND CONCENTRATION LIMIT OF TRYPTOPHAN AND GLUCOSE

by

Allison Schulkins

APPROVED FOR THE DEPARTMENT OF BIOMEDICAL, CHEMICAL AND MATERIALS ENGINEERING

SAN JOSÉ STATE UNIVERSITY

December 2017

Melanie McNeil, Ph.D.

Liat Rosenfeld, Ph.D.

Anand Ramasubramanian, Ph.D.
Department of Biomedical, Chemical and Materials Engineering

Department of Biomedical, Chemical and Materials Engineering

Department of Biomedical, Chemical and Materials Engineering 


\title{
ABSTRACT \\ A FULL FACTORIAL ANALYSIS OF POLYMERIZATION CONDITIONS OF SODIUM ALGINATE BEADS TO DETERMINE THE EFFECT ON DIFFUSIVITY AND CONCENTRATION LIMIT OF TRYPTOPHAN AND GLUCOSE
}

\author{
by Allison Schulkins
}

The purpose of this work is to develop an experimental setup with associated mathematical analysis to analyze the changes in diffusivity through calcium alginate gel beads made under different polymerization conditions. The diffusivity of the solutes glucose and tryptophan was calculated by measuring the concentration change over time as solute diffused out of the alginate spheres and into the bulk. The alginate beads were generated using different polymerization conditions following a two-level, three-factor, full-factorial matrix with alginate concentration, cross-linker concentration, and crosslinking time as the factors. The diffusivities showed variations between different conditions, which were analyzed to determine the main effects. No factors or interactions were found to affect the diffusivity of tryptophan. The main effects on glucose diffusivity were the cross-link time and the interaction of alginate concentration and cross-linker concentration. The initial concentration absorbed by the gel matrix showed variation across different polymerization conditions. Statistical analysis was repeated for the initial concentration. Cross-linking time and the interaction between cross-link concentration and time were the main effects on glucose initial concentration, and no factors influenced tryptophan initial concentration. 


\section{ACKNOWLEDGEMENTS}

Many semesters of work by senior students in ChE 162L have been spent testing new variables to develop these experiments. Their time spent in taking data and developing methods to analyze the output has provided me with the framework to build this study. Dr. McNeil has guided the progress of this work, and her support and insights have shaped the focus of this study.

For personal support and understanding, special thanks to my husband Will Schulkins. His patience is unmatched and his eagerness to learn is infectious. 


\section{TABLE OF CONTENTS}

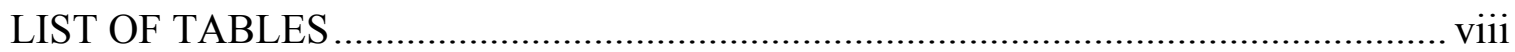

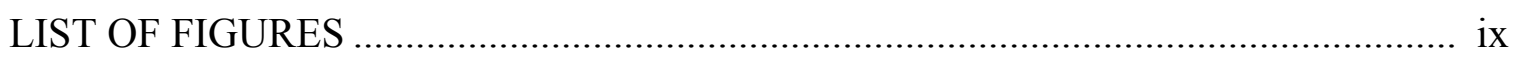

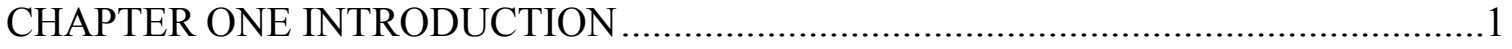

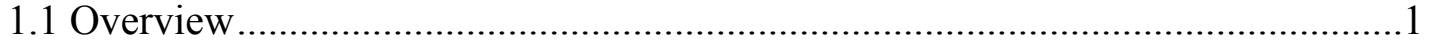

1.2 Significance

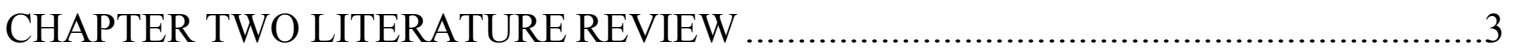

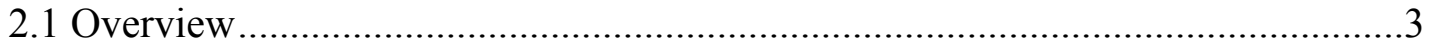

2.2 Structure of and Applications Using Alginate Beads ...........................................

2.3 Variations in Polymerization Conditions............................................................

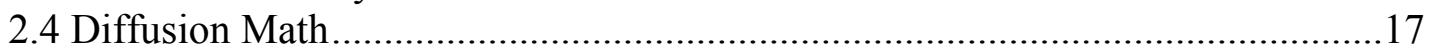

2.5 Statistical Analysis and Design of Experiments ................................................20

2.6 Literature Review Summary ……………………….....................................22

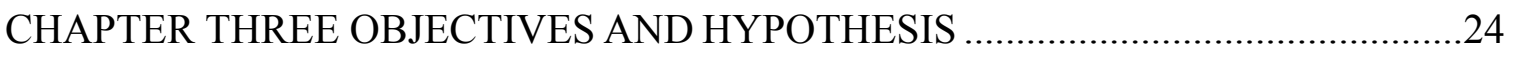

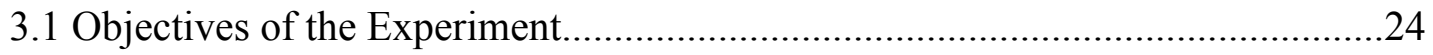

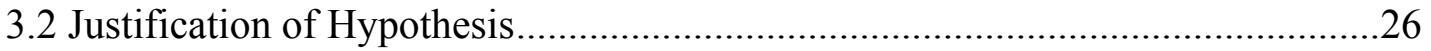

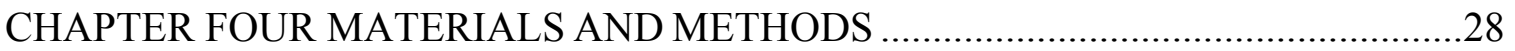

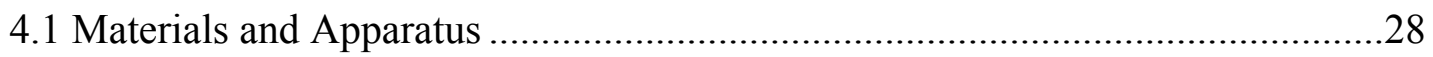

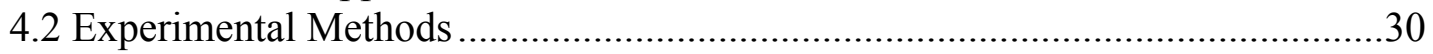

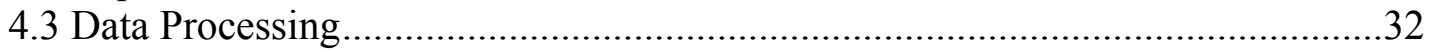

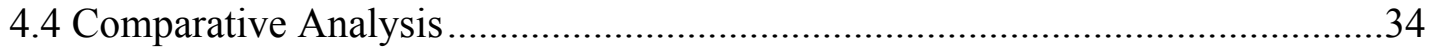

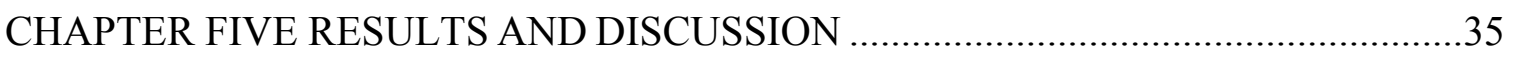

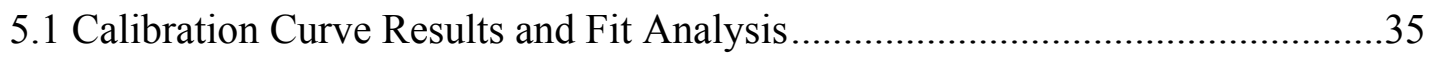

5.2 Bead Diameter Data and Discussion..................................................................37

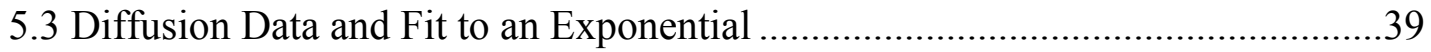

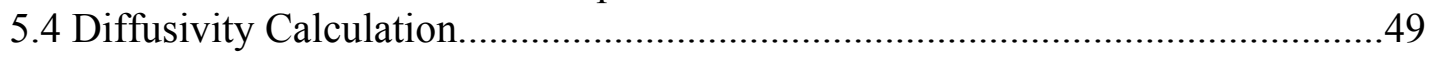

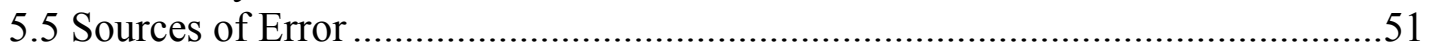

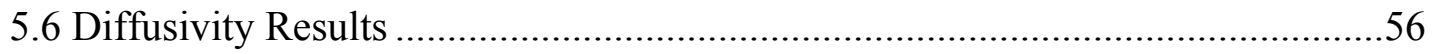

5.7 Discussion of Diffusivity Results ..................................................................63

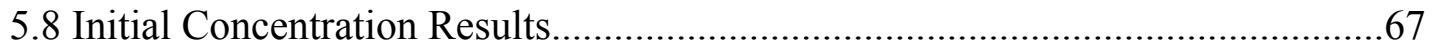

5.9 Discussion of Initial Concentration Results.........................................................74

5.10 Recommendations for Design of Experiments .................................................77 


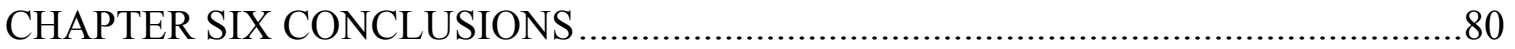

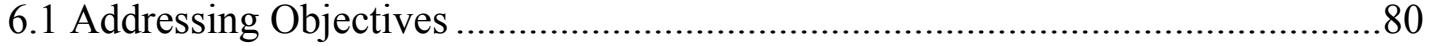

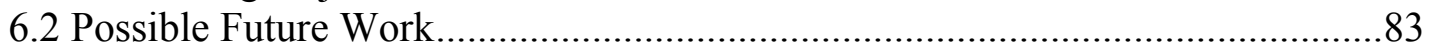

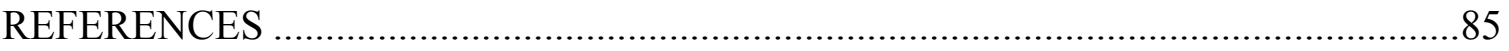

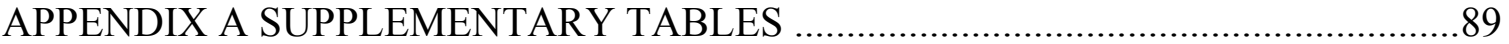

APPENDIX B RECOMMENDED EXPERIMENTAL PROTOCOL ..............................93 


\section{LIST OF TABLES}

Table 1 Calculated mean effects on different responses in a $2 \mathrm{v}^{5-1}$ fractional

Table 2 Alginate polymerization factorial matrix ......................................... 30

Table $3 \quad$ Summary of sampling conditions for each solute ….......................... 31

Table 4 Average of calculated diffusivity for both tryptophan and glucose ......... 50

Table 5 Stokes radius for glucose and tryptophan, calculated using Equation 7... 66

Table 6 Initial concentration for both tryptophan and glucose ........................... 69 


\section{LIST OF FIGURES}

Figure 1 Structure of alginic acid with M-, G-, and MG blocks......................... 5

Figure 2 Equatorial slices of alginate beads incubated in fluorescently labeled immunoglobulin $\mathrm{G}$

Figure 3 Scanning electron microscopy (SEM) images of calcium alginate gel beads.

Figure 4 "Egg-box" model diagram with proposed configuration of nitrophenol contaminates taken up by alginate beads.

Figure 5 Response curve showing diffusion coefficients of RG19-dextran (MW $42.5 \mathrm{kDa})$ diffusing through gels.

Figure 6 Concentration measured over time for various solutes diffusing from the bulk fluid into $\mathrm{Ca}$-alginate beads.

Figure 7 Concentration measured over time for various solutes diffusing out of Ca-alginate beads into the bulk.

Figure 8 Tryptophan calibration curve of known concentrations versus absorbance taken at $280 \mathrm{~nm}$

Figure 9 Glucose calibration curve of known concentrations versus absorbance taken at $500 \mathrm{~nm}$

Figure 10 Average diameter for beads measured with a micrometer taken after diffusion trials for tryptophan and glucose runs

Figure 11 All measured bead diameters for all runs for each bead sample

Figure 12 Tryptophan time test taken using bead sample A, measuring concentration of tryptophan released from the beads and into the bulk ...

Figure 13 Glucose time test taken using bead sample A, measuring concentration of glucose released from the beads and into the bulk

Figure 14 Diffusion data collected for all tryptophan trials

Figure 15 Diffusion data collected for the first glucose trial 
Figure 16 Diffusion data collected for the second glucose trial

Figure 17 Residuals for general exponential fit of tryptophan diffusion data .......... 46

Figure 18 Residuals for general exponential fit of run 1 glucose diffusion data ...... 47

Figure 19 Residuals for general exponential fit of run 2 glucose diffusion data ...... 48

Figure 20 Ratio of standard deviation and the average of five absorbance values plotted versus time for all tryptophan data points

Figure 21 Ratio of standard deviation and the average of five absorbance values plotted versus time for all glucose data points

Figure 22 Effects and interactions on diffusivity of tryptophan and glucose............ 56

Figure 23 Prediction profile for tryptophan diffusivity created using JMP .............. 57

Figure 24 Prediction profile for glucose diffusivity created using JMP ................... 58

Figure 25 Half-normal probability plot of tryptophan diffusivity effects and interaction

Figure 26 Half-normal probability plot of glucose diffusivity effects and interactions

Figure 27 Factors and interactions of glucose and tryptophan on initial concentration, $\mathrm{C}_{0}$

Figure 28 Prediction profile for tryptophan initial concentration created using JMP

Figure 29 Prediction profile for glucose initial concentration created using JMP ... 71

Figure 30 Half-normal probability plot of tryptophan initial concentration, $\mathrm{C}_{0} \ldots . . .72$

Figure 31 Half-normal probability plot of glucose initial concentration, $\mathrm{C}_{0} \ldots \ldots \ldots \ldots . \quad 72$ 


\section{CHAPTER ONE}

\section{INTRODUCTION}

\subsection{Overview}

Alginate is a linear polysaccharide composed of monomers 1-4' linked $\beta$-Dmannuronate $(\mathrm{M})$ and $\alpha$-L-guluronate $(\mathrm{G})$. The $\mathrm{G}$ units can bind to divalent cations, cross-linking the linear chains and forming a hydrogel [1-3] almost instantaneously. The hydrogel formed from sodium alginate cross-linked with calcium ions has been used for encapsulation of cells, drug release, and other biomedical applications [4,5]. The crosslinked alginate is appealing as a material for these applications because it is biocompatible and inexpensive, while still allowing diffusion of small molecules.

Many variables can affect the membrane properties of the cross-linked alginate. For example, the ratio of $\mathrm{M}$ units and $\mathrm{G}$ units can affect the density [1], the cross-linking ions change its physical properties $[6,7]$, and cross-linker concentration can affect the mass transfer ability of solutes from the material [8]. There are methods of experimental design [9-11] that can be used to unravel which of these and other variables are the main factors influencing a response. Statistical analysis paired with a design of experiments (DOE) approach gives quantitative measures and confidence in determining the most important factors. A DOE approach has been applied to diffusion studies through alginate gels $[12,13]$. These examples demonstrate the power of DOE by revealing interactions between factors that can have profound impacts on the mass transfer properties of the membrane. 


\subsection{Significance}

Optimizing the membrane composition for mass transfer is critical for certain applications. Applying a DOE approach to polymerization of alginate will allow polymers to be custom-made to suit the application. For cell encapsulation, mass transfer of many solutes through the membrane is critical for the viability of the cells. In drug delivery applications with alginate, mass transfer of the drug needs to be carefully controlled to meet the specifications for delivery. These two examples highlight the significance of using a systematic approach for studying mass transfer in alginate membranes. Furthermore, it is possible that the membrane composition inhibits diffusion of the solute, making certain applications (e.g. drug release) difficult. Polymerization conditions of alginate have been varied in previous studies $[7,8,12,14]$ and stiffness, porosity, and diffusion rate have been measured. Some previous work has focused on changes in solute diffusivity [15-17] with different alginate polymerization conditions.

The focus of this study is the calculation of diffusivity of tryptophan and glucose as they diffuse out of alginate polymer spheres and into bulk fluid. The experimental procedures and analysis presented intends to be an outline for studying how changes in polymerization of alginate influence the diffusivity. In particular, the senior undergraduate lab course in the chemical engineering department at San José State University has been using iterations of this experiment. The work presented in this study can serve as a much-needed outline for the lab course. For future experiments in the senior undergraduate lab, students can adapt this experimental setup to use different factors, levels, and solutes. 


\section{CHAPTER TWO \\ LITERATURE REVIEW}

\subsection{Overview}

Alginate is used for a range of applications including encapsulation, drug delivery, and heavy metal sequestration. It has been shown in many studies that changing polymerization conditions affects the membrane's mass transfer properties. The changes in mass transfer properties in alginate polymers are dependent on the solute. DOE has been used to show statistically significant factors and interactions affecting mass transfer.

Diffusion is described by Fick's $2^{\text {nd }}$ Law, which can be modified for spherical coordinates. Crank's book [18] develops equations modeling diffusion for many different system geometries. The book develops an equation describing the concentration change over time of a solute diffusing out of a sphere in the radial direction. This equation can be applied to a system of solute-saturated alginate spheres in a bulk fluid initially free of solute.

Statistics and the DOE approach are used in combination to determine the main effects in an experiment. This approach is a natural starting point to optimizing the polymers for alginate diffusion experiments. Combining DOE with analysis of variance (ANOVA) can find the most effective variables for facilitating diffusion and calculating the confidence of the measurements.

\subsection{Structure of and Applications Using Alginate Beads}

Alginate is a copolymer of $\mathrm{M}$ units ( $\beta$-D-mannuronate) and $\mathrm{G}$ units ( $\alpha$-L-guluronate) extracted from brown algae. The ratio of $\mathrm{M} / \mathrm{G}$ units in the alginate affects the strength of 
the gels created [19]. Different species of algae and the method of alginate extraction produce different ratios of $\mathrm{M} / \mathrm{G}$ units [20]. Polymerization of alginate is accomplished by the $\mathrm{G}$ units complexing with divalent cations. Grant et al. [2] first proposed in 1973 that the polymer-cation complex forms cooperatively as an "egg-box". The egg-box model is a way to visualize the gelation in alginate, likened to a corrugated box for storing eggs. The $\mathrm{G}$ units interact with each other, using the cation as a bridge. Many linear alginates form a regular structure around the cations. The cations are left in the interstitial channels like eggs sitting in a box. This simple two-dimensional model has provided a template for understanding the chemical interactions of alginate. Of course, this gelation occurs in all three dimensions. The egg-box model has proven to be a useful tool in mapping out the chemical interactions within alginate gels in the years since it was first proposed.

The structure of alginate has allowed applications in heavy metal sequestration and removal of toxic substances from water. Papageorgiou et al. [3] tested a hypothesis that the hydroxyl group on the $\mathrm{M}$ and $\mathrm{G}$ units can increase the affinity of alginate to metal ions like copper and cadmium. They found that alginate had a high uptake capacity for these metal ions compared to other low cost adsorbents. The researchers credit the high $\mathrm{M} / \mathrm{G}$ ratio of the alginate used in their experiments for it high uptake capacity. The eggbox model proposed by Grant et al. [2] outlined that the G units are responsible for the gelation of alginate by the cross-linking ions. Figure 1 shows the conformation changes between different subunits in a long alginate chain, as reported by Papageorgiou et al. [3]. The conformation of the G-Block is oriented to allow cross-linking ions to travel to 
interstitial regions and form a weak bond between two saccharide groups. The research of Papageorgiou et al. also demonstrated that the diffusion coefficient for copper and cadmium ions was independent of initial concentration of the solutes. This is consistent with theory: the diffusion coefficient or diffusivity is independent of initial concentration.

\section{GMMMMGGGGGGGGGGMGMGMGMMMMMG}

\section{M-Block G-Block MG-Block M-Block}

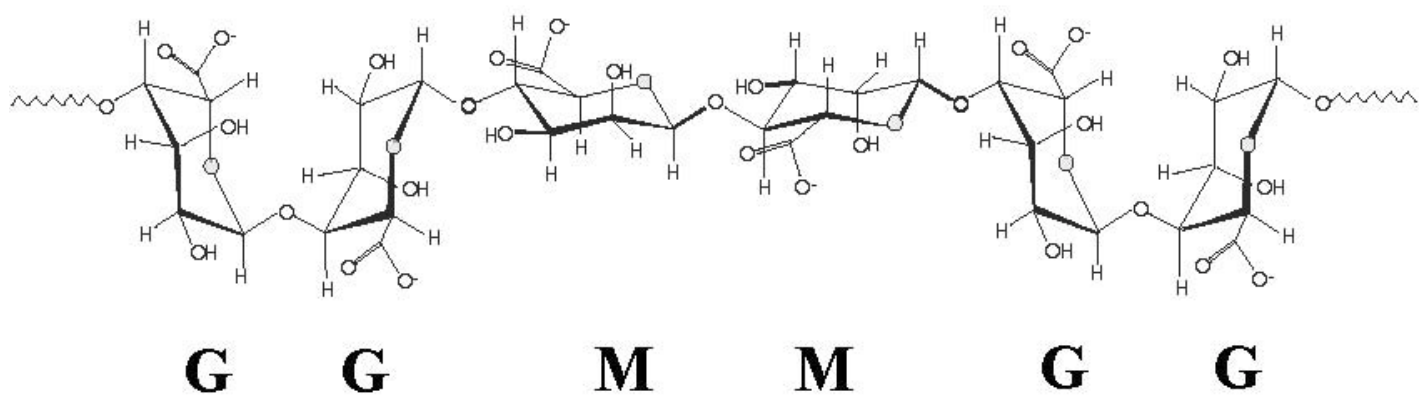

Figure 1. Structure of alginic acid with M-, G-, and MG blocks. Reprinted with permission from Elsevier [3].

Polymerization conditions for alginate are biologically safe. This makes alginate an appealing material for encapsulation of biomolecules, bacteria, or other cells. Wang et al. [4] encapsulated embryonic stem cells with a high level of cell viability. In order for encapsulated cells to be viable in alginate, diffusion across the membrane needs to be free to allow for exchange of metabolic materials for the cells. The results from Wang et al. show that the alginate membrane did not impede the diffusion of solutes for the cells. The ability of solutes to diffuse through alginate membranes can be broken down into three categories, according to a review on the subject by Stewart [20]. First, ionic solutions diffuse well, but are likely adsorbed in the matrix of the membrane. Since the 
ions are adsorbed on the membrane, these ions are excluded from the encapsulated cells. Second, smaller nonpolar solutes (defined as having a molecular weight lower than 44 Daltons) diffuse well and are available for encapsulated cells. Third, large solutes with a molecular weight greater than 44 Daltons diffuse poorly or not at all, becoming effectively unavailable to encapsulated cells. So, as long as the cell does not need to secrete or uptake high molecular weight solutes, encapsulation in alginate is attractive to simplify purification and reduce cell clumping.

Alginate has been studied to use as a material for slow release of drugs or molecules. Castro et al. [5] compiled a review of work on the slow release of drugs in $\beta$-glucans and emulsan biopolymers. Emulsan-alginate microspheres showed sensitivity under specific pHs and temperatures, leaving possibilities for tuning to specific physiological conditions for drug delivery. In a review on drug delivery systems by Tønnesen and Karlsen [21], drugs are encapsulated in the alginate matrix by either spray coating or encapsulating a solid or liquid suspension containing the drug. The release of the drug is controlled by careful preparation of the alginate matrix. For this reason, diffusion studies of the drug through the alginate matrix must be done to ensure controlled release. These studies each demonstrate that alginate membranes can be adjusted to the mass transfer applications.

\subsection{Variations in Polymerization Conditions}

Many studies have shown that changing the conditions of alginate polymerization gives the membrane different strength, mass transfer properties, and size. Alginate is traditionally cross-linked using calcium ions, due to its low cost and biocompatibility. However, gelation is possible using other divalent cations. Using different cations has 
also been shown to affect the properties of the alginate membrane. MØrch et al. [6] created alginate gel beads using calcium chloride, barium chloride, and strontium chloride and visualized beads in a confocal microscope after incubating with fluorescently labeled immunoglobulin $\mathrm{G}(\mathrm{IgG})$. Their results are presented in Figure 2. Their data is a striking visual example of the changes in alginate permeability that are possible under different polymerization conditions. Using different cross-linking ions at different concentrations yielded different distributions of $\operatorname{IgG}$ across the radial direction of the sphere. In particular, the lowest concentration of barium chloride solution (Figure 2B) yielded the most non-homogeneous distribution of fluorescence intensity, with the highest signal on the edge of the sphere. In other words, the IgG did not penetrate into the center of the alginate bead. It is interesting to note in this example that none of the conditions presented have a homogeneous fluorescent signal across the radial direction. 
A
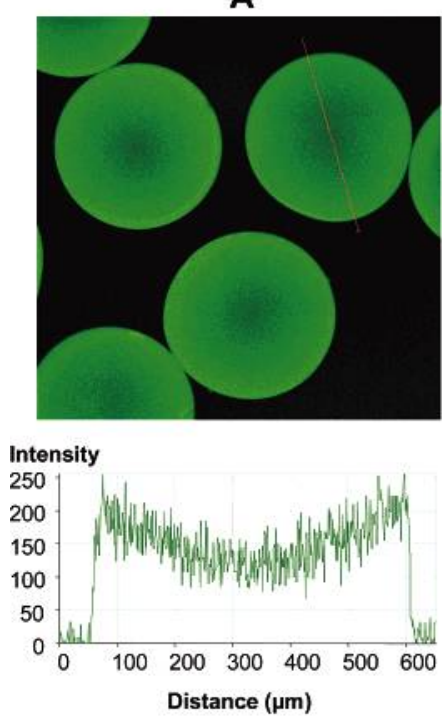

D

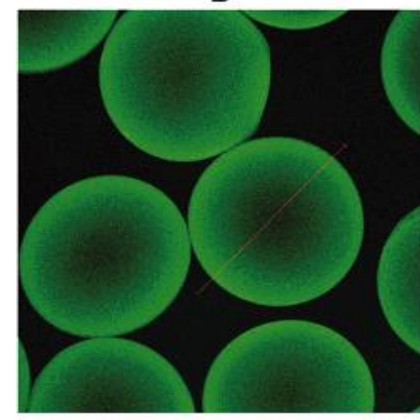

Intensity

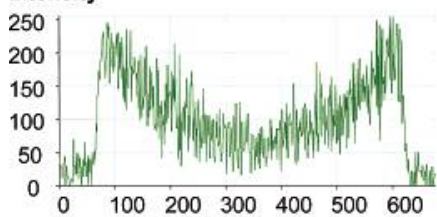

Distance $(\mu \mathrm{m})$
B
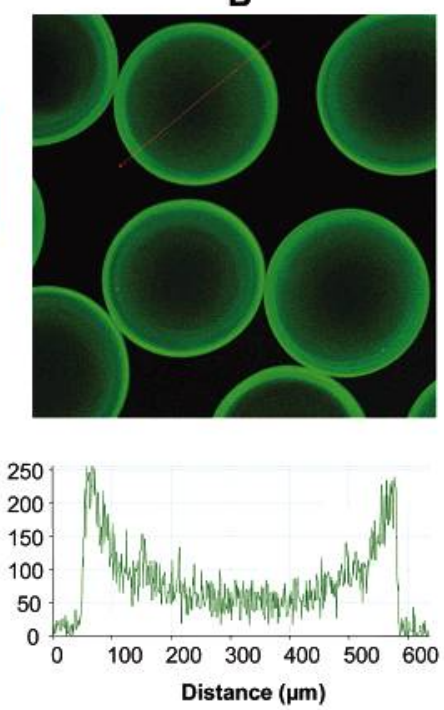

E
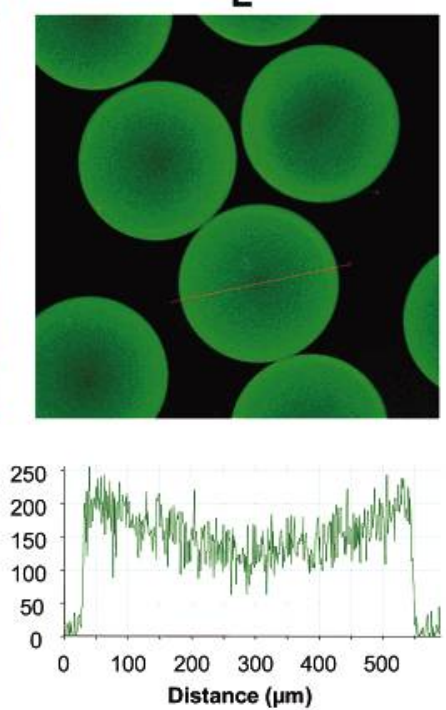

Figure 2. Equatorial slices of alginate beads incubated in fluorescently labeled immunoglobulin $\mathrm{G}$, visualized using a confocal scanning laser microscope. Beads were polymerized using A) $50 \mathrm{mM} \mathrm{CaCl2,} \mathrm{B)} 10 \mathrm{mM} \mathrm{BaCl2,} \mathrm{C)} 20 \mathrm{mM} \mathrm{BaCl2,} \mathrm{D)} 20 \mathrm{mM}$ $\mathrm{CaCl}$, E) SrCl2. Reprinted with permission from reference [6]. Copyright (2006) American Chemical Society.

These data in Figure 2 were collected using high-G alginate, which could contribute to the occlusion of IgG at the center, given that more $\mathrm{G}$ units have been reported to lead to a tighter gel matrix [3]. Additionally, IgG is a fairly small protein, but this

phenomenon would likely be less problematic when using a smaller molecule as a solute. 
It is, however, important to note that concentration is not uniform in the radial direction, which could change the mathematical modeling of the system.

Aslani and Kennedy [7] compared diffusion in alginate gel films cross-linked with calcium and zinc ions. Permeability between calcium or zinc alginate films was not found to be significantly different. However, changes in gelling time and cation concentration showed variations in permeability. In particular, the permeability was reduced by almost four-fold with a two-fold increase of cation concentration. Their findings also report a dramatic drop in permeability in response to increasing cross-link time. It is reasonable that higher concentration of cations can cross-link the membrane into a tighter network due to $\mathrm{G}$ units interacting with the high concentration of cations. This tighter network could impede mass transfer, depending on the solute. Similarly, cross-linking the membrane for a longer time could allow the membrane more time to be cross-linked to a denser network of pores, further hindering mass transfer through the membrane. It should be noted that these studies use the word permeability, which is a common word used in membrane mass transfer studies. To be clear, in these cases the permeability measured in MØrch et al. [6] and Aslani and Kennedy [7] is synonymous with diffusivity, since the solute moves across the membrane with a concentration gradient and not due to some pressure gradient.

Peretz and Cinteza [22] studied alginate hydrogels to remove nitrophenol derivatives from wastewater. The Langmuir and Freundlich isotherm models were used to model the adsorption of nitrophenols on the polymer matrix. The alginate hydrogel spheres in their experiments were made using varied concentrations of calcium chloride from 0.01 to $5 \%$ 
$(\mathrm{w} / \mathrm{v})$. Their findings show that $0.5 \% \mathrm{w} / \mathrm{v}$ calcium chloride was the minimal salt concentration where the solubility in their isotherm was unaffected. The cross-link times were varied between 10 minutes and 24 hours. The effect of cross-link time was not expounded further in their published results, except to report that equilibrium was reached by 24 hours. Under these polymerization conditions, Peretz and Cinteza observed the removal of the nitrophenols within the first 12 hours of incubation. The shape and morphology of the alginate beads was characterized using scanning electron microscopy (SEM). Figure 3 shows that the surface of the alginate was found to be quite rough, covered in hills and valleys.

(a)

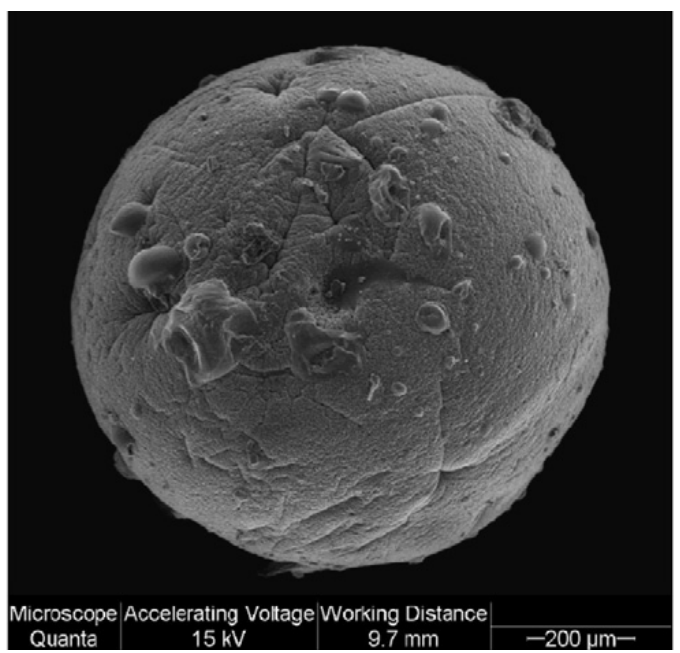

(b)

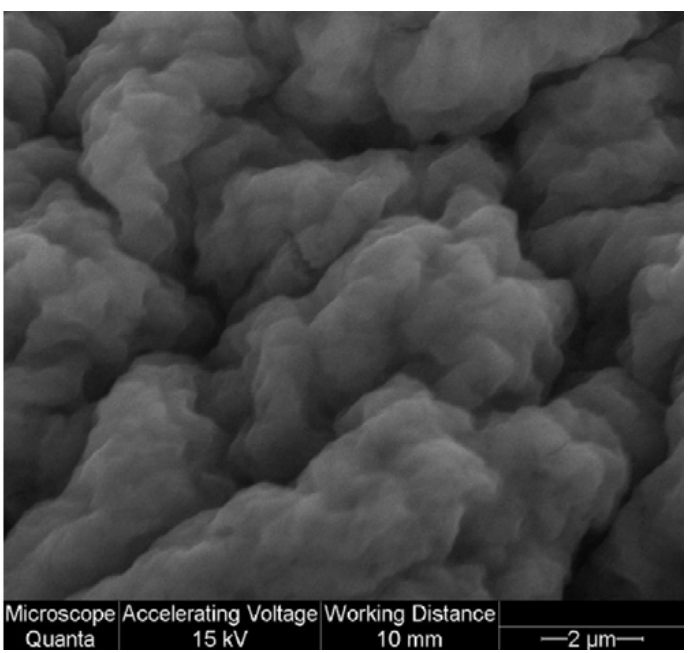

Figure 3. Scanning electron microscopy (SEM) images of calcium alginate gel beads, a) alginate bead b) surface image showing porosity. Reprinted with permission from Elsevier [22].

Peretz and Cinteza [22] generated an egg-box model to describe the chemical interactions of the nitrophenols with the alginate membrane. This schematic is presented in Figure 4. The carboxyl groups on the alginate chains interact with the nitrophenols, effectively trapping them inside the gel matrix. This model demonstrates the chemical 
nature of the alginate membrane. It is possible that other solutes would not diffuse through the alginate gel membrane due to unfavorable chemical interactions.

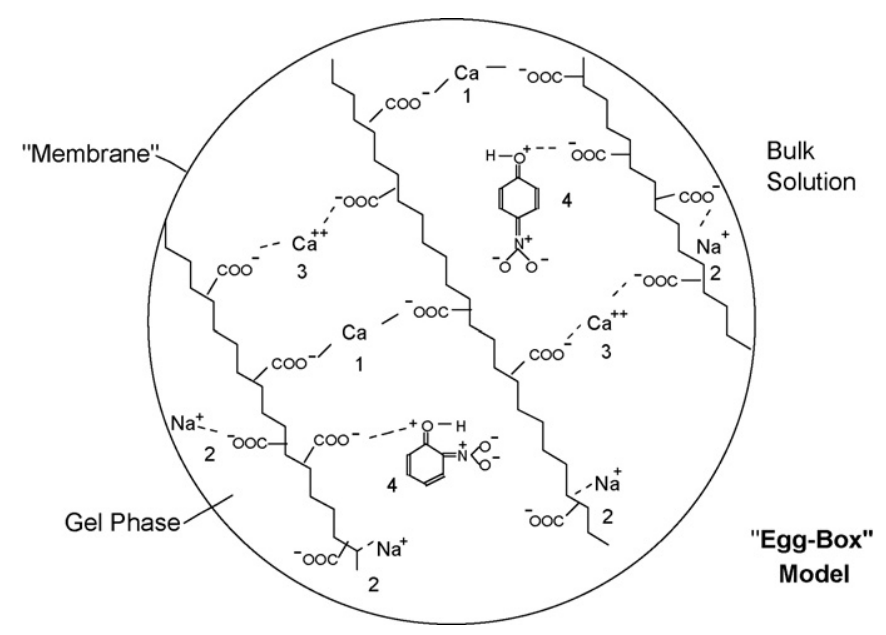

Figure 4. "Egg-box" model diagram with proposed configuration of nitrophenol contaminates taken up by alginate beads. Reprinted with permission from Elsevier [22].

Chan et al. [14] measured Young's Modulus and bead diameter for alginate beads made with varied alginate concentration, cation concentration, and $\mathrm{M} / \mathrm{G}$ ratio. Their data shows that a higher Young's Modulus was measured when the alginate had a high degree of cross-linking. In particular, a low $\mathrm{M} / \mathrm{G}$ ratio, higher alginate concentration, and larger cations all resulted in higher measured Young's Modulus. Donati et al. found similar results [23] studying alginate swelling and its effect on Young's Modulus. Mitchell and Blanshard [24] observed this same phenomenon in their study on alginate swelling. In all of these studies, a high $\mathrm{M} / \mathrm{G}$ ratio yielded a weaker and more elastic alginate gel. Mitchell and Blanshard's research showed that alginate gels swell due to calcium ions exchanging with the solution, meaning the cross-linking with calcium was not permanent. Building on their work, Donati et al. observed increased swelling when alginate was 
incubated in saline solution. Bajpai and Sharma [25] studied this swelling in their work comparing calcium and barium ions for cross-linking alginate. Their findings showed that calcium alginate had $160 \%$ water uptake compared to $40 \%$ in barium alginate. This swelling would make mathematical modeling of alginate beads difficult due to changes in the radius of the spheres.

The design of experiments approach has been used for alginate experiments by Østberg et al. [12] and Holte et al. [13]. Østberg et al. performed a fractional factorial experimental matrix varying the cross-linker concentration, cross-linking time, alginate concentration, amount of drug dispersed in the cross-linking solution, and the $\mathrm{M} / \mathrm{G}$ ratio. Their goal was to study the drug delivery from the alginate beads. Table 1 shows their effects and interactions on many responses; most notably the column titled "Drug content" refers to the amount of drug dispersed from the alginate beads. This is the most analogous to mass transfer properties that was measured in their study. The drug content remaining in the beads would be a measure of how much drug was not delivered. The results in Table 1 show that the alginate concentration, cross-linker concentration, and amount of drug dispersed significantly impacted the amount of drug in the matrix of the membrane. An increase in calcium concentration or alginate concentration led to worse mass transfer. This makes sense in theory, as a higher cross-linker concentration results in a tighter membrane, which could impede diffusion. The same is true for increasing alginate concentration. The amount of drug dispersed refers to the concentration of drug that was dispersed into the bead-making suspension. Not surprisingly, more drug added to the alginate beads led to more drug leaving the membrane. Essentially, this variable 
increased the concentration gradient across the membrane, and the results are expected.

This initial concentration of drug did affect the amount of drug dispersed by the

membrane, but diffusion is independent of the initial concentration of solute [3].

Table 1. Calculated mean effects on different responses in a $2 \mathrm{v}^{5-1}$ fractional factorial matrix. Reprinted with permission from Elsevier [12].

\begin{tabular}{|c|c|c|c|c|c|c|}
\hline \multirow[t]{2}{*}{ Factor/interaction } & \multicolumn{3}{|c|}{ Effects on } & \multirow[b]{2}{*}{$\begin{array}{l}\text { Moisture } \\
\text { content } \\
(\%)\end{array}$} & \multirow[b]{2}{*}{$\begin{array}{l}t_{50 \%} \\
(\mathrm{~min})\end{array}$} & \multirow[b]{2}{*}{$\begin{array}{l}t_{80 \%} \\
(\min )\end{array}$} \\
\hline & $\begin{array}{l}\bar{d}_{\max } \\
(\mathrm{mm})\end{array}$ & $\begin{array}{l}\text { Drug } \\
\text { content } \\
(\%)\end{array}$ & $\begin{array}{l}\text { Calcium } \\
\text { content } \\
(\%)\end{array}$ & & & \\
\hline A: calcium concentration & $-0.14^{a}$ & $-2.8^{a}$ & $1.15^{\mathrm{a}}$ & -0.1 & $19^{a}$ & $138^{a}$ \\
\hline B: gelling time & -0.02 & -1.1 & $0.20^{\text {a }}$ & -0.8 & $9^{a}$ & 25 \\
\hline C: alginate concentration & $0.14^{a}$ & $-7.6^{a}$ & -0.08 & 0.9 & 2 & $35^{\circ}$ \\
\hline D: amount of drug dispersed & 0.05 & $28.0^{a}$ & $-1.98^{a}$ & $-5.3^{a}$ & $24^{a}$ & $77^{a}$ \\
\hline E: alginate type (low $\mathrm{G} /$ high $\mathrm{G}$ ) & 0.02 & -0.3 & 0.13 a & 0.6 & $13^{a}$ & $80^{\text {a }}$ \\
\hline Interaction $\mathrm{AB}$ & 0.00 & 0.6 & -0.33 & 0.4 & 3 & -7 \\
\hline Interaction $\mathrm{AC}$ & -0.03 & 0.3 & 0.10 & -0.1 & $12^{\mathrm{a}}$ & $85^{\mathrm{a}}$ \\
\hline Interaction $\mathrm{AD}$ & 0.02 & -0.3 & $-0.30^{a}$ & 0.6 & $12^{a}$ & $36^{\circ}$ \\
\hline Interaction AE & 0.00 & 0.3 & $0.15^{a}$ & -0.7 & 3 & $43^{a}$ \\
\hline Interaction $B C$ & 0.02 & 0.1 & 0.00 & 0.2 & $6^{a}$ & 20 \\
\hline Interaction $\mathrm{BD}$ & 0.01 & 0.3 & $-0.20^{a}$ & 0.9 & $7^{\mathrm{a}}$ & $59^{\mathrm{a}}$ \\
\hline Interaction $\mathrm{BE}$ & 0.00 & 0.3 & 0.00 & 0.1 & $8^{a}$ & 25 \\
\hline Interaction CD & -0.03 & $-3.2^{a}$ & $0.33^{a}$ & 0.4 & 0 & -12 \\
\hline Interaction $\mathrm{CE}$ & -0.05 & 0.1 & -0.03 & 0.2 & 2 & $32^{a}$ \\
\hline Interaction $\mathrm{DE}$ & 0.01 & 0.8 & -0.03 & -0.1 & $10^{a}$ & $43^{a}$ \\
\hline
\end{tabular}

a Significant, $\alpha=0.01$.

Holte et al. [13] also used a design of experiments approach and generated response curves showing the impact of alginate concentration, cross-linker concentration, and $\mathrm{M} / \mathrm{G}$ ratio on the diffusivity. Their study found that quinine was able to diffuse freely through the pores of the matrix. However, the higher molecular weight dextran (MW $42.5 \mathrm{kDa}$ ) had a diffusion rate too slow to be observed. The response curve for dextran is shown in Figure 5. The highest diffusivity from the response curve occurs at the lowest concentration of alginate, the higher $\mathrm{G}$ content, and lowest calcium concentration. It is possible that the dextran is unable to diffuse into the gel matrix at all, or some other mass transfer phenomena are at work, but was not suggested in their work. The only factor 
that was deemed to have an effect on the diffusion of both solutes was the cross-linker concentration. The study also observed an interaction between the alginate concentration and the $\mathrm{M} / \mathrm{G}$ ratio, where the effect of $\mathrm{M} / \mathrm{G}$ ratio was less pronounced when alginate concentration was at the lowest level. These results and the results in Table 1 show that interactions between factors can be easily revealed using DOE.

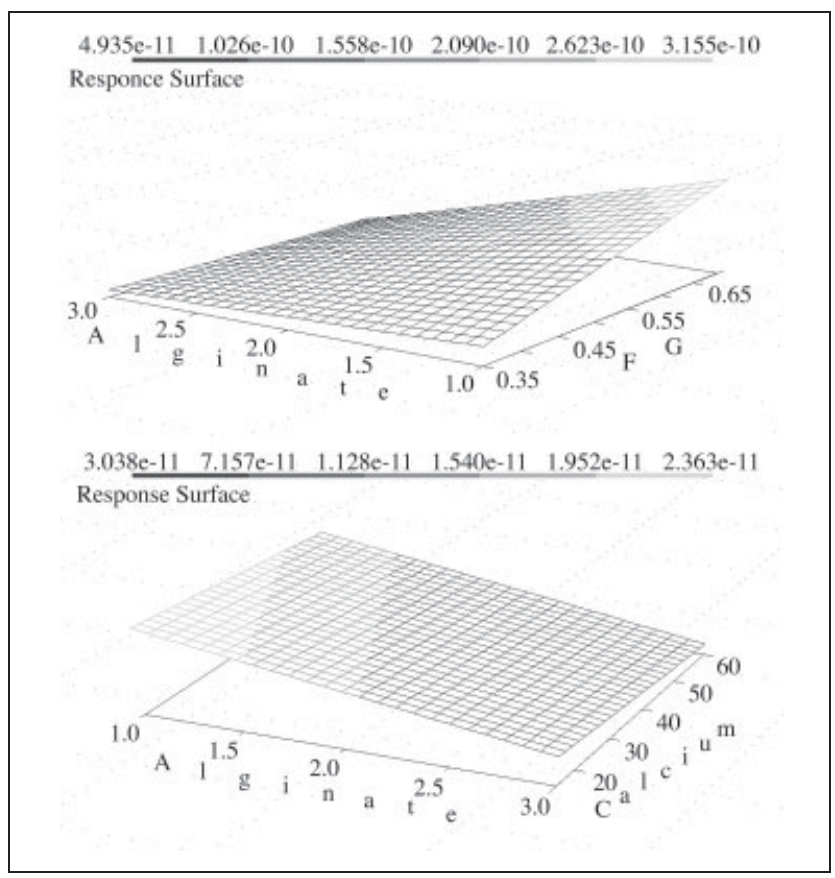

Figure 5. Response curve showing diffusion coefficients of RG19-dextran (MW 42.5 $\mathrm{kDa}$ ) diffusing through gels of different alginate concentration, $\mathrm{M} / \mathrm{G}$ ratio $(\mathrm{FG})$ and calcium concentration (in $\mathrm{mM}$ ). Reprinted with permission from Pharmazie [13].

It is possible that the alginate membrane inhibits mass transfer. For example, Figure 6 shows the drop in concentration of three different solutes as they diffuse into alginate beads from the bulk, as reported by Tanaka et al. [15]. Tryptophan diffusion into the alginate beads is completed quite fast compared to the lactoalbumin and albumin. Within 30 minutes, the tryptophan reaches the equilibrium concentration. The paper reports the 
diffusion coefficient for tryptophan as the same as the diffusion of tryptophan in water.

Tanaka et al. conclude that tryptophan is able to diffuse freely through the gel.

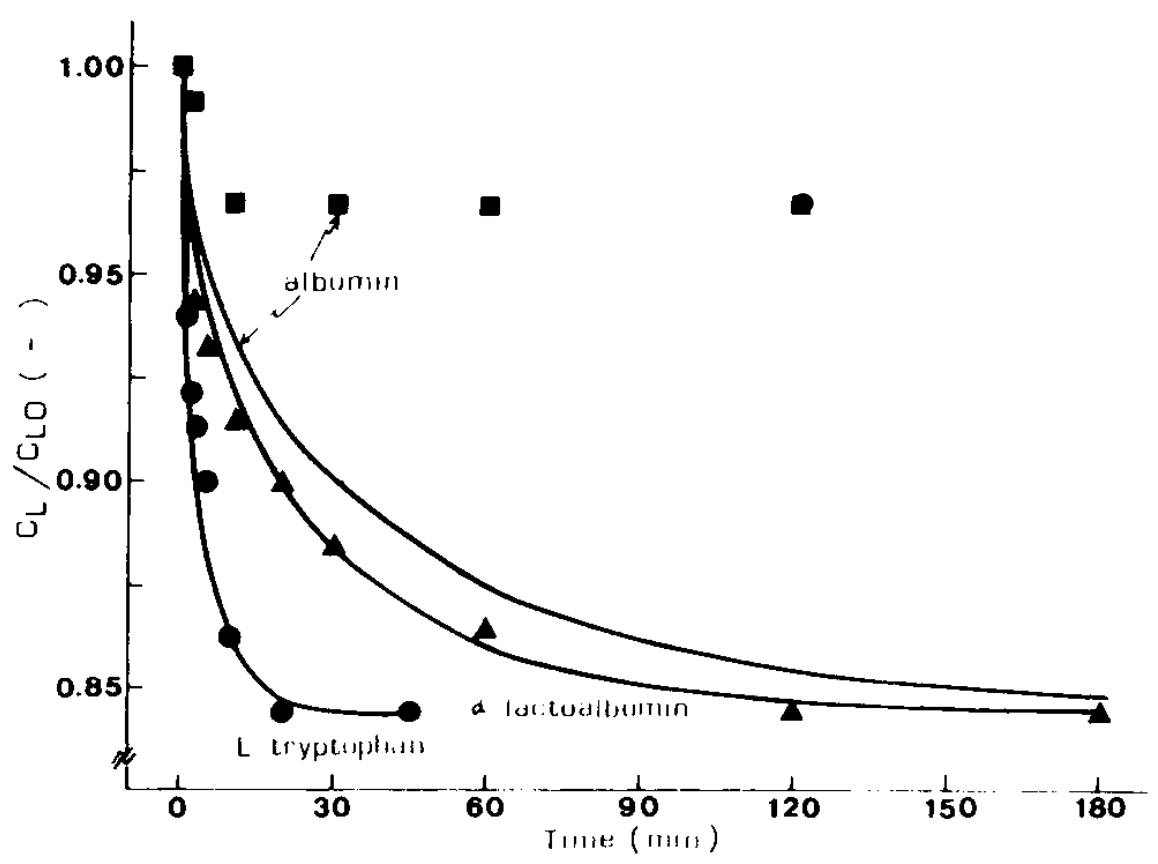

Figure 6. Concentration measured over time for various solutes diffusing from the bulk fluid into Ca-alginate beads. Solutes reported are L-tryptophan, lactoalbumin, and albumin. Reprinted with permission from reference [15]. Copyright (1984) John Wiley and Sons.

Tanaka et al. also measured diffusion out of the alginate beads. Mixing the solute into the alginate solution before cross-linking ensured that the initial concentration was the same for all beads. The beads were incubated in a fluid initially free of solute and the solute was allowed to diffuse into the bulk fluid over time. The change in concentration over time was used to calculate the diffusivity for glucose, lactoalbumin, and albumin. The change in relative concentration over time for all three solutes is presented in Figure 7. From the data, glucose diffuses very fast and shows stable equilibrium in less than one hour. Lactoalbumin reaches an equilibrium concentration by 3 hours. Albumin did not 
measurably diffuse through alginate until 4 hours of incubation. These results from Tanaka et al. show glucose and tryptophan to be attractive solutes, given their short diffusion time. However, the data reported suggests that the alginate matrix did not impede mass transfer. In fact, the diffusivity calculated in the study was equal to the diffusivity of the solutes in water. However, it has been shown that mass transfer can be affected by changing the alginate polymerization conditions [12,13]. Therefore, it is possible that alginate can impede the diffusion of glucose and tryptophan, but was not observed in the experimental conditions that Tanaka et al. used in their study.

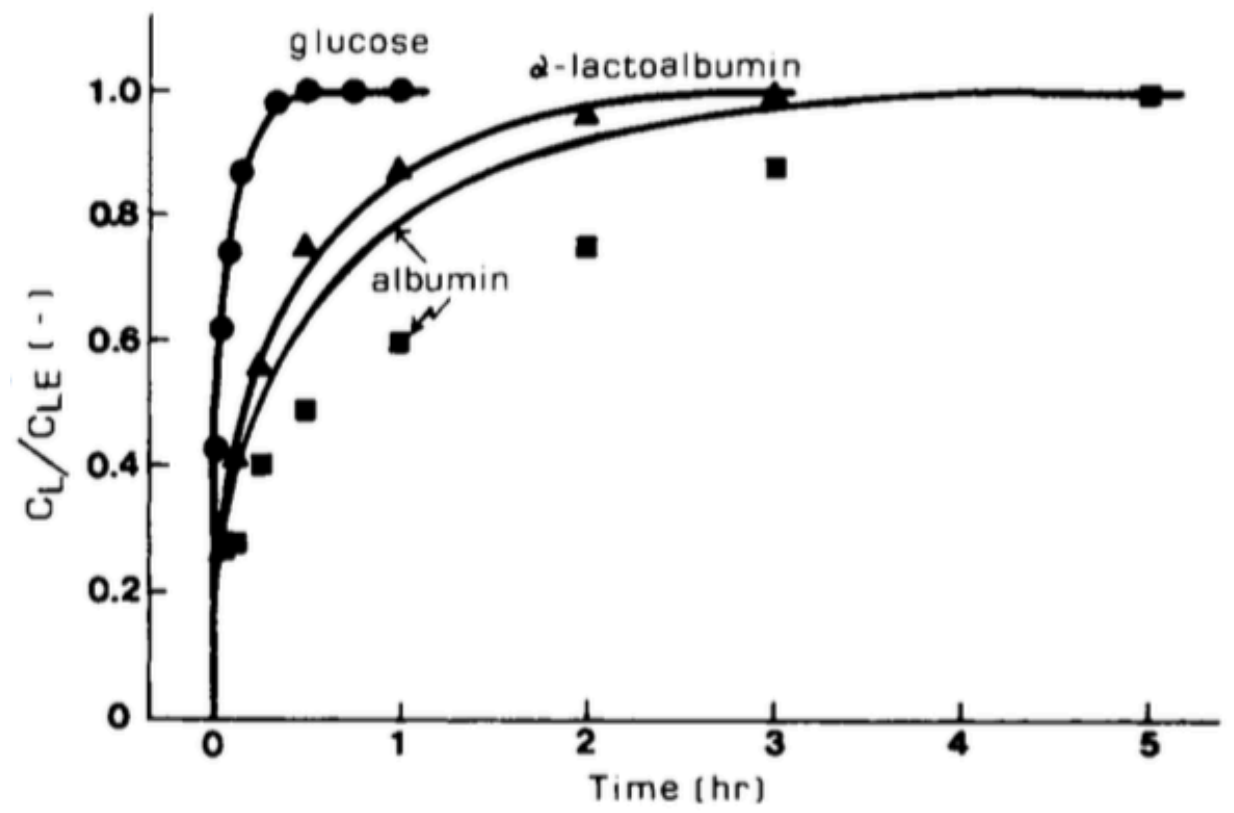

Figure 7. Concentration measured over time for various solutes diffusing out of Caalginate beads into the bulk. Reprinted with permission from reference [15]. Copyright (1984) John Wiley and Sons.

Especially for research related to clinical applications like drug delivery or encapsulation of cells, optimization of the polymerization conditions will lead to more repeatable mass transfer properties in alginate membranes. It is clear that the specific 
polymerization conditions may work well for one solute, but may be unsuitable for another due to size, charge density, or other reasons. The levels need to be tested to find the optimal conditions for the solute of study. Many studies focus on diffusion or adsorption as the only mass transfer phenomena occurring in the system. This is rarely proven or addressed, and ignores the possibility of reactions in the system.

\subsection{Diffusion Math}

Equation 1 shows the general form for Fick's $2^{\text {nd }}$ law in spherical coordinates, which describes the change in concentration, $\mathrm{C}$, over time, t, due to diffusion [26] where $\mathrm{D}$ is diffusivity.

$$
\frac{\partial C}{\partial t}=\frac{1}{r^{2}}\left\{\frac{\partial}{\partial r}\left(D r^{2} \frac{\partial C}{\partial r}\right)+\frac{1}{\sin \theta} \frac{\delta}{\partial \theta}\left(D \sin \theta \frac{\partial C}{\partial \theta}\right)+\frac{D}{\sin ^{2} \theta} \frac{\partial^{2} C}{\partial \phi^{2}}\right\}
$$

Equation 1

In many systems, Equation 1 is simplified by assuming negligible diffusion in the theta and phi directions, leaving only diffusion radially, in the $\mathrm{r}$ direction. This assumption can be justified by symmetrical geometry in the system. Diffusion from the center point of a sphere would have symmetrical diffusion in the theta and phi directions. Assuming all measurable mass transfer by diffusion occurs only in the radial direction, Equation 1 is simplified by crossing out all terms containing theta or phi. Crank [18] develops a solution for Equation 1 describing the concentration of a solute diffusing out of a sphere into a well-stirred solution that is initially free of solute. The equations developed by Crank have been used in literature for experiments studying diffusion in or out of alginate spheres. For example, Ha, Engler, and Lee [16] used Crank's text to calculate the diffusion coefficient of solute into alginate spheres for the purpose of designing a bioreactor. Tanaka et al. [15] also used equations developed in Crank's book to compare 
the diffusion coefficients of many solutes into and out of alginate spheres. To develop the equation used in these studies, Crank first established a ratio of volume in solution versus the volume of the sphere. This ratio is called $\alpha$, and is shown in Equation 2.

$$
\alpha=\frac{3 V}{4 \pi a^{3}}
$$

Equation 2

In Equation 2, $\mathrm{V}$ is the volume of solution, a is the radius of the sphere, assuming the radius is constant. It should be noted that given the observations of Donati et al. [23], Mitchell and Blanshard [24], and Bajpai and Sharma [25], this assumption may not be sound for alginate beads due to swelling. Equation 2 finds the ratio of the bulk solution versus each sphere, which models the entire system as diffusion from a single sphere. Equation 3 describes the concentration at a known time, $\mathrm{C}_{\mathrm{t}}$, due to diffusion. The initial concentration of solute in the sphere is $\mathrm{C}_{0}, \mathrm{D}$ is the diffusivity, and $\mathrm{t}$ is time.

$$
C_{t}=\frac{C_{0}}{1+\alpha}\left\{1-\sum_{n=1}^{\infty} \frac{\left(6 \alpha(1+\alpha) \exp \left(-D q_{n}^{2} t / a^{2}\right)\right)}{9+9 \alpha+q_{n}^{2} \alpha^{2}}\right\}
$$

Equation 3

Equation 3 models a system where concentration of the bulk fluid is measured over time. Crank assumes that the concentration of the bulk is equal to the concentration at the surface of the spheres. Equation 3 assumes that the spheres have a uniform initial concentration, $\mathrm{C}_{0}$, and the boundary layer on the outer surface of the spheres is negligible. This assumption is reasonable as long as the solution is well mixed. Many of the research on mass transfer through alginate polymer does not stress or mention the importance of mixing. If the fluid is not well mixed, these equations cannot be accurate in modeling the system, as a concentration boundary layer term would need to be 
considered. Equation 3 also assumes diffusion is negligible in the phi and theta directions. The $\mathrm{q}_{\mathrm{n}}$ terms are found by solving for the nonzero roots of Equation 4 .

$$
\tan q_{n}=\frac{3 q_{n}}{3+\alpha q_{n}}
$$

Equation 4

Solutions for $\mathrm{q}_{\mathrm{n}}$ can be found for many values of alpha in Table 6.1 of Crank's textbook [18]. Lastly, if the initial concentration inside the sphere is not known, Equation 5 is needed. Equation 5 relates the ratio of volumes, $\alpha$, to the mass of the solute in the bulk fluid at equilibrium, $\mathrm{M}_{\infty}$.

$$
\frac{3 M_{\infty}}{4 \pi a^{3} C_{0}}=\frac{1}{1+1 / \alpha}
$$

Equation 5

Using Equation 2 to find alpha, the initial concentration, $\mathrm{C}_{0}$, can be solved from Equation 5. Diffusivity can then be solved using Equation 3 for a system where concentration is measured over time.

In a membrane system, the size of the solute is critical for mass transfer. The Stokes radius can be useful when comparing two or more molecules to be an approximation of size. It assumes the solute molecule is a hard sphere of radius $r$. Equation 6 shows the Stokes-Einstein equation, where $\mathrm{D}$ is the diffusivity, $\mathrm{k}_{\mathrm{B}}$ is the Boltzmann Constant, $\mathrm{T}$ is the temperature in Kelvin, $r$ is the Stokes radius, and $\eta$ is the dynamic viscosity of the solvent.

$$
D=\frac{k_{B} T}{6 \pi r \eta}
$$

Equation 6

The Stokes-Einstein equation [26] can be rearranged to find the Stokes radius, r, related to the molecular weight of the molecule. Equation 7 shows this rearranged form, where 
MW is the molecular weight of the molecule, $\mathrm{N}$ is Avogadro's number, and $\rho$ is the solvent density.

$$
r=\left[\frac{3(M W)}{4 \pi N \rho}\right]^{1 / 3}
$$

Equation 7

\subsection{Statistical Analysis and Design of Experiments}

Statistics are needed to calculate error and add confidence to calculated and measured values in order to draw conclusions from data. Statistical analysis also helps when comparing the output from an experimental matrix. To study the influence of different factors on a response, a DOE approach can be used to create an experimental matrix. Factorial experimental design often results in fewer total experimental runs needed compared to changing one variable at a time. Interactions between different variables, called factors, can be found with DOE. These interactions would not be known by changing one factor at a time [9]. The number of levels refers to the number of values of a factor (e.g. a low and high concentration of alginate is two levels and a low, middle, and high temperature is three levels, etc.). The number of factors and levels determines the size of the final experimental matrix. The number of runs for a full-factorial experiment can be found using Equation 8, or raising the number of levels by the number of factors.

\section{Number of Runs $=$ Number of levels ${ }^{\text {Number of factors }} \quad$ Equation 8}

The downstream analysis of the DOE results can also identify the most important factors that influence the response, called a main effect. In a two-level DOE, the effect of a factor is determined by the average of the response values for runs where the factor was 
high $\left(\mathrm{Y}_{+}\right)$subtracted by the average of the response values for runs where the factor was low (Y.), or as outlined in Equation 9 [9].

$$
\text { Effect }=\bar{Y}_{+}-\bar{Y}_{-}
$$

Equation 9

Effects calculated using Equation 9 from an experiment would be compared. Positive effects facilitate the response and negative effects hinder the response. For an interaction, if the calculated effect is positive, those factors positively impact the response. Effects or interactions calculated close to zero do not have significant impact on the response, according to this method.

To determine which effects and interactions are significant or not due, a normal probability plot is often used [10]. A normal probability plot is a graphical method to determine if the data follows a normal distribution. The effects for all factors and interactions, calculated using Equation 9, are sorted in descending order and plotted against the medians of a standard normal distribution. If the plot is linear, then all effects are due to normal error. If one or more data points are off the line, then those factors likely vary outside of normal error. A normal probability plot is a quick visual way to see factors that affect the response outside of normal error. A half-normal probability plot can also be used [9]. The half-normal plot adds an improvement on the normal probability plot by ranking the absolute value of the effects. This makes the half-normal probability plot independent of the positive or negative signs assigned for each level.

In statistics, experimental data is often analyzed by an analysis of variance, or ANOVA. ANOVA is often presented in a table to show the statistical impact of the factors in the experimental matrix [27]. ANOVA makes three assumptions: observations 
are independent, residuals are randomly distributed, and variances between groups are the same. ANOVA tests the null hypothesis [11], which is that the means of two groups are the same. If the probability, or P-value, is shown to be low enough (often the threshold is $5 \%$ or $1 \%$ ), then the null hypothesis is rejected and the effect is considered statistically significant. In other words, if the variation between two groups has a low probability of being due to normal error, then their variance is likely due to their experimental variables. Pairing ANOVA with a full-factorial experimental design can show the statistical impact of experimental factors and verify main effects calculated using Equation 9.

\subsection{Literature Review Summary}

In summary, work on alginate polymers has a long history and many applications. In general, the literature consensus is that the alginate polymer gets physically tougher with a higher degree of cross-linking. The mass transfer through the alginate membrane tends to decrease with a higher degree of cross-linking. The degree of cross-linking is affected by many variables. The cations involved in the polymerization, the cation concentration, the alginate concentration, the $\mathrm{M} / \mathrm{G}$ ratio of alginate, and the cross-linking time have all been shown to have an effect on cross-linking of the polymer.

To measure the diffusion of a solute out of a sphere and into bulk solution, Crank [18] developed Equation 3 from Fick's $2^{\text {nd }}$ Law. The equation could be solved to find the diffusivity if all other variables are known. To compare solutes in a mass transfer system, Stokes radius can be used as a simplified size comparison by modeling the molecules as a hard sphere based on their molecular weights. 
A DOE approach is a way to plan experiments that can reduce the total number of runs compared to changing one variable at a time. This method can find the main effects and interactions between factors, which would never be known by changing one factor at a time. DOE begins with planning the experimental matrix and ends with statistical analysis. This approach is invaluable in a system like alginate polymerization, where there are many possible factors that influence a response. 


\section{CHAPTER THREE}

\section{OBJECTIVES AND HYPOTHESIS}

\subsection{Objectives of the Experiment}

The purpose of this study was to perform a full factorial analysis of the polymerization conditions of sodium alginate beads to determine the effects on the diffusivity and initial concentration of tryptophan and glucose. To accomplish this purpose, four objectives were set. First, a standard experimental setup for generating acceptably uniform spherical alginate beads was established. This includes finding appropriate levels for alginate, cross-linker concentration, and cross-link time that work well for the experiment. The second objective was to measure the concentration versus time of solutes diffusing out of the polymers made under different conditions. A full factorial experimental matrix was made to test the effect of polymerization conditions on diffusion. The change in concentration over time was used to calculate diffusivity and initial concentration. The third objective in this work was to outline the mathematical pathway for calculating diffusivity that is appropriate to the geometry of the system. The fourth and final objective in this work is to determine the most important factors that influence diffusivity and initial concentration, so that recommendations can be made for future experiments.

Alginate concentration, cross-linker concentration, and cross-link time were chosen as factors because they have all been studied previously in the literature. They are all operationally easy to change. With these factors, all of the experimental variations occur in the alginate polymerization. This means that the mass transfer data can be collected 
under identical conditions, because the variations are in the membrane composition. To avoid issues with changing radius in the calculations, the experiment was designed to allow the beads to reduce the possibility of swelling during data collection. The alginate beads were incubated in a high concentration of solute overnight. The salt content of this solution was similar to the solution used during data collection. Therefore, if swelling were to affect the bead radius, it would occur during the overnight incubation and would be less likely to change size during the data collection.

This experimental setup and method of analysis will be used to refine an existing experiment in the senior undergraduate chemical engineering lab course. Students can reference this study to vary polymerization conditions or study different solutes in experiments of their own design. Design of experiments and solutions of non-linear differential equations are an important part of the chemical engineering curriculum. This alginate experiment provides students with a relatively easy and inexpensive way to gain experience with these mathematical analysis techniques while studying a system that has relevant applications for their future careers.

The hypothesis of this study is that varying the polymerization conditions of alginate concentration, cation concentration, and cross-linking time will affect the diffusivity of glucose and tryptophan. A full-factorial experimental matrix was developed using DOE techniques to test this hypothesis. Diffusivity of glucose and tryptophan was measured out of alginate beads. It was proposed that the diffusivity would decrease with higher concentrations of alginate, cation concentration, and increased cross-linking time. 


\subsection{Justification of Hypothesis}

Many published studies on alginate show that physical properties and permeability change according to polymerization conditions of the alginate membrane. Alginate concentration has been shown by Chan et al. [14] and Tanaka et al. [15] to affect Young's modulus and diffusivity, respectively. This seems to be due to stiffer beads, as measured by Chan et al. [14]. A higher concentration of alginate leads to more closely packed linear polymers. This yields a tight pore network when they are cross-linked. Østberg et al. [12] also found that changes in alginate concentration significantly impacted the dispersion of drug in their system. Therefore, alginate concentration is an attractive factor for diffusion studies.

Chan et al. [14] found that an increase in cation concentration led to a higher Young's Modulus. It is suggested in this study that a stiffer bead will decrease diffusivity due to a tighter network in the polymer matrix, impeding diffusion of solute. Presumably, a higher concentration of cross-linker would increase the degree of cross-linking in the gel because more cations are available to interact with $\mathrm{G}$ units. This seems to be supported by the literature. Aslani and Kennedy [7] compared cation concentration in gel beads made with calcium and zinc ions and found that in both beads, permeability decreased with increasing cation concentration. Holte et al. [13] found the cation concentration to be the only important factor affecting diffusivity in their design of experiments approach. These studies all provide support for choosing cation concentration as a factor in a mass transfer experiment. Therefore, it was chosen as a second candidate, along with alginate concentration, to use in this study. 
Cross-linking time was studied in Aslani and Kennedy's work [7] and shown to decrease permeability in the gel. However, the most dramatic change in permeability measured in their study was comparing cross-linking time of 1-2 minutes to 5 minutes. After 5 minutes of gelation time, the permeability through their membranes did not change significantly. Østberg et al. [12] also including gelling time in their experimental matrix testing drug dispersion in the alginate membrane. The impact of gelling time was not statistically significant on the drug dispersion for the levels tested. Due to the ambiguous findings from Aslani and Kennedy, cross-linking time was chosen as the third and final factor to vary in this study of polymerization and diffusion. Cross-linking time also has the advantage of being economical as an experimental variable: no expensive reagents need to be purchased to test this factor. 


\section{CHAPTER FOUR}

\section{MATERIALS AND METHODS}

\subsection{Materials and Apparatus}

Alginate beads were made using alginic acid sodium salt from brown algae at medium viscosity by Sigma-Aldrich. The alginate solutions were made using deionized water and added drop-wise using a plastic syringe (Thermo Scientific) into the crosslinking solution. Cross-linking solution was made using calcium chloride from SigmaAldrich. The solutes used in this work were D (+)-Glucose from Acros and LTryptophan from Sigma-Aldrich. Glucose samples were measured by diluting 100-fold with pre-warmed Glucose Oxidase Reagent (Pointe Scientific, Inc.), then incubating for 5 minutes at $37^{\circ} \mathrm{C}$ to allow the colored product to develop.

Absorbance was measured using an Agilent 8453A UV-Vis System along with an Agilent Technologies UV quartz cell cuvette for solutes that absorb in the UV spectrum (tryptophan) and plastic cuvettes suitable for the visible spectrum (glucose) from Fisher Scientific. Once samples were taken and diffusion runs were finished, the radius of 5 beads from each alginate bead population were measured using a micrometer by Swiss Precision Instruments.

\subsection{Experimental Methods}

A stock solution of each solute was made in deionized water for glucose or Tris buffer at $\mathrm{pH} 8.6$ in the case of tryptophan. Glucose was diluted 100-fold with glucose oxidase reagent and incubated at $37^{\circ} \mathrm{C}$ for 5 minutes to obtain absorbance at $500 \mathrm{~nm}$. This is according to manufacturer recommendations of the glucose oxidase reagent. 
Glucose oxidase reagent produces a colored product in the presence of glucose. The absorbance of the colored product is proportional to the glucose concentration in the sample. Tryptophan absorbs light directly at $280 \mathrm{~nm}$, so extra reagents were not needed. Calibration curves were made for each solute using a serial dilution of the stock solutions and plotting the known solute concentration versus the corresponding absorbance. All glucose measurements in the calibration curve use the known concentration of glucose, not 100-fold diluted, to directly correlate absorbance from the colored product with the concentration of glucose in the sample. Solute concentration of unknown samples during diffusion runs was calculated by doing a linear regression of the solute's calibration curve, as defined by the Beer-Lambert Law.

Alginate solutions of $1.5 \% \mathrm{w} / \mathrm{v}$ and $2.5 \% \mathrm{w} / \mathrm{v}$ were made in deionized water. Alginate beads were formed by adding alginate drop-wise to a cross-link solution of calcium chloride (either $5 \% \mathrm{w} / \mathrm{v}$ or $10 \% \mathrm{w} / \mathrm{v}$ ) and incubating for a cross-link time (either 15 minutes or 60 minutes), as outlined in Table 2. Beads were then washed three times in $0.9 \% \mathrm{w} / \mathrm{v}$ sodium chloride solution and stored at $4{ }^{\circ} \mathrm{C}$ in the sodium chloride solution until ready for overnight incubation with a concentrated solute solution. The full factorial set of alginate beads were made twice to test reproducibility. The radius of 5 randomly selected beads was measured with a micrometer on the day of data collection. 
Table 2. Alginate polymerization factorial matrix.

\begin{tabular}{|l|l|l|l|}
\hline $\begin{array}{l}\text { Sample } \\
\text { Name }\end{array}$ & $\begin{array}{l}\text { Alginate Solution } \\
(\% \text { alginate w/v) }\end{array}$ & $\begin{array}{l}\text { Cross-link solution } \\
(\% \mathrm{CaCl} 2 \mathrm{w} / \mathrm{v})\end{array}$ & $\begin{array}{l}\text { Cross-link time } \\
(\text { minutes })\end{array}$ \\
\hline A & $1.5 \%$ & $5 \%$ & 15 \\
\hline B & $2.5 \%$ & $5 \%$ & 15 \\
\hline C & $1.5 \%$ & $10 \%$ & 15 \\
\hline D & $2.5 \%$ & $10 \%$ & 15 \\
\hline E & $1.5 \%$ & $5 \%$ & 60 \\
\hline F & $2.5 \%$ & $5 \%$ & 60 \\
\hline G & $1.5 \%$ & $10 \%$ & 60 \\
\hline H & $2.5 \%$ & $10 \%$ & 60 \\
\hline
\end{tabular}

Sample times were determined prior to any diffusion runs using a pilot run for each solute wherein the speed of diffusion of a solute was determined by taking regular samples every 30 seconds. The concentration versus time data were plotted for the pilot run, and sampling times were chosen for each solute by choosing time ranges where the slope was the highest. More samples were required during the time range where the highest concentration changes occur, and fewer data points were required once the sample approached equilibrium. In order to assume a constant volume, less than $10 \%$ total volume was to be removed from the total solution due to sampling. Therefore, the smallest volume needed for the cuvettes was taken and the pilot run served as a benchmark for flagging the important time points. Minimizing volume removal and selectively choosing sampling times based on diffusion speed was part of planning the number of samples taken and the time points to use in the experiment.

To take diffusion data, a measured volume of alginate beads were added to a solution with very high concentration of solute and incubated overnight in closed $50 \mathrm{~mL}$ tubes. The concentrations for the overnight solution were $40 \mathrm{mg} / \mathrm{mL}$ for glucose and 0.33 $\mathrm{mg} / \mathrm{mL}$ for tryptophan. On the day of data collection, the tube of alginate beads was 
drained of the overnight solution and the beads were added to an empty beaker. A volume of water (or Tris buffer in the case of tryptophan) that was ten times the measured volume of alginate beads was added to the beaker with the stir bar mixing the solution. A timer was started at the moment the bulk solution was added. Samples were removed with a pipette at sampling times determined by the pilot run and absorbance measured as outlined, depending on the solute. Glucose was run twice with two independently made sets of alginate beads. Table 3 summarizes the sampling conditions for each solute. Depending on the cuvette used for a solute and the dilution needed, sampling volume varied as outlined. In the case of glucose, recovered samples were mixed in a ratio of 1 to 100 with Glucose Oxidase Reagent, warmed in a $37^{\circ} \mathrm{C}$ water bath for 5 minutes, and measured at $500 \mathrm{~nm}$ in plastic cuvettes with the visible light option selected on the spectrophotometer. Tryptophan samples were measured by direct absorbance at $280 \mathrm{~nm}$ on the spectrophotometer in a quartz cuvette with the UV light selected. Every sample absorbance was measured five times on the spectrophotometer to obtain standard deviation in the instrument.

Table 3. Summary of sampling conditions for each solute.

\begin{tabular}{|l|l|l|}
\hline & Glucose & Tryptophan \\
\hline Sample volume $(\mu \mathrm{L})$ & 60 & 150 \\
\hline Dilution factor & 100 & 0 \\
\hline Diluted with & $\begin{array}{l}\text { Glucose Oxidase } \\
\text { at } 37^{\circ} \mathrm{C}\end{array}$ & Tris buffer, $\mathrm{pH} 8.6$ \\
\hline Measured at $(\mathrm{nm})$ & 500 & 280 \\
\hline
\end{tabular}




\subsection{Data Processing}

Measured absorbance for each solute and sample of beads was converted to the equivalent concentration using the calibration curves. Solute concentration versus time data was plotted for each solute and each bead sample. The average and standard deviation for all five absorbance measurements at each data point was calculated, as well as the ratio of standard deviation to the average concentration.

The equation to describe diffusion of solute out of spheres (Equation 3) is an exponential function. A fit of the experimental diffusion data to a general exponential function was used to calculate the asymptote. The asymptote of the concentration over time data is the equilibrium concentration, $\mathrm{C}_{\infty}$. The general exponential function, Equation 10, was used to fit the concentration and time points for all diffusion trials, where $\mathrm{a}, \mathrm{k}$, and $\mathrm{b}$ are constants.

$$
f(x)=a e^{-k t}+b
$$

The constants were found using Mathematica 10.2 and the fit was verified to be good by calculating the $\mathrm{R}^{2}$ and residuals for each diffusion trial. The limit as time went to infinity gave the equilibrium concentration, $\mathrm{C}_{\infty}$, for each diffusion trial. This equilibrium concentration is needed to solve for diffusivity in Equation 3. The mass of solute at equilibrium ( $\mathrm{M}_{\infty}$, in Equation 5) was calculated by multiplying equilibrium concentration by the total volume of the bulk.

To determine variation of alginate bead size, the diameter of the alginate beads was measured with a micrometer from 5 randomly chosen beads at the end of each diffusion trial. The averages and standard deviations for the measured diameters were calculated, 
as well as the ratio of standard deviation versus the average. These values were calculated and compared for each run and also across bead samples of similar polymerization conditions. The volume of the bulk solution per alginate bead was calculated by solving for the number of beads in each diffusion trial, assuming them to be packed in a random packing fraction for spheres [28]. The radius and volume per alginate bead was used in Equation 2 to calculate alpha for each diffusion trial. The initial concentration in the beads was calculated by solving for $\mathrm{C}_{0}$ in Equation 5 .

To solve for diffusivity of each trial, a solver using the Goal Seek function across many cells of data in Microsoft Excel was written to solve for D in Equation 3. The Excel solver varied the diffusivity term, $D$, until the $C_{t}$ term in Equation 3 matched the measured concentration. Equation 3 assumes that the concentration boundary layer on each sphere is negligible, and so a stir plate was used with the same setting for all trials to ensure good mixing. Equation 3 also assumes the initial concentration in the spheres to be uniform at $\mathrm{C}_{0}$, which was calculated using Equation 5. The diffusivities for each trial were averaged and the standard deviation calculated, as well as the ratio between the average and the standard deviation. Each trial had a calculated diffusivity for each time point. Data points were excluded if the difference between a calculated diffusivity and the average over the entire trial was more than one standard deviation. Upon exclusion of errant data points, an average diffusivity was calculated to be the consensus diffusivity for all runs. 


\subsection{Comparative Analysis}

A full-factorial analysis was done to compare the responses of diffusivity and initial concentration $\left(\mathrm{C}_{0}\right)$ between all runs of each solute. Diffusivity is the subject of this study, so the full-factorial analysis was done to show how polymerization conditions of alginate affect diffusivity of these two solutes. Initial concentration was also used as a response because the starting concentration in the alginate sphere was found to be not uniform across the different bead samples. It is proposed in this study that the membrane matrix could be different enough affect the concentration of solute allowed to equilibrate with the membrane in the overnight solution. Initial concentration of solute has been shown to be independent of diffusivity [3], but studying this variable as a response could inform more about the concentration limit of the polymers.

The main effects and interactions for both responses were compared. A prediction profile and half-normal probability plot was used to visualize the calculated effects [9]. Analysis of variance (ANOVA) was used to calculate the main factors and interactions impacting the responses. ANOVA statistics, including sum of squares, degrees of freedom, $\mathrm{F}$ ratio, and the probability for the $\mathrm{F}$ value, were obtained using JMP. 


\section{CHAPTER FIVE}

\section{RESULTS AND DISCUSSION}

\subsection{Calibration Curve Results and Fit Analysis}

Figures 8 and 9 show the calibration curves for tryptophan and glucose, respectively. Each sample was measured five times on the spectrophotometer and the average is plotted. A linear regression on the standard curve was performed. The $\mathrm{R}^{2}$ was found to be over 0.9 for both calibration curves, which is more than adequate to be considered a good fit. The equation for the lines obtained from the calibration curves was used to calculate the concentration of unknown solute in the mass transfer experiments.

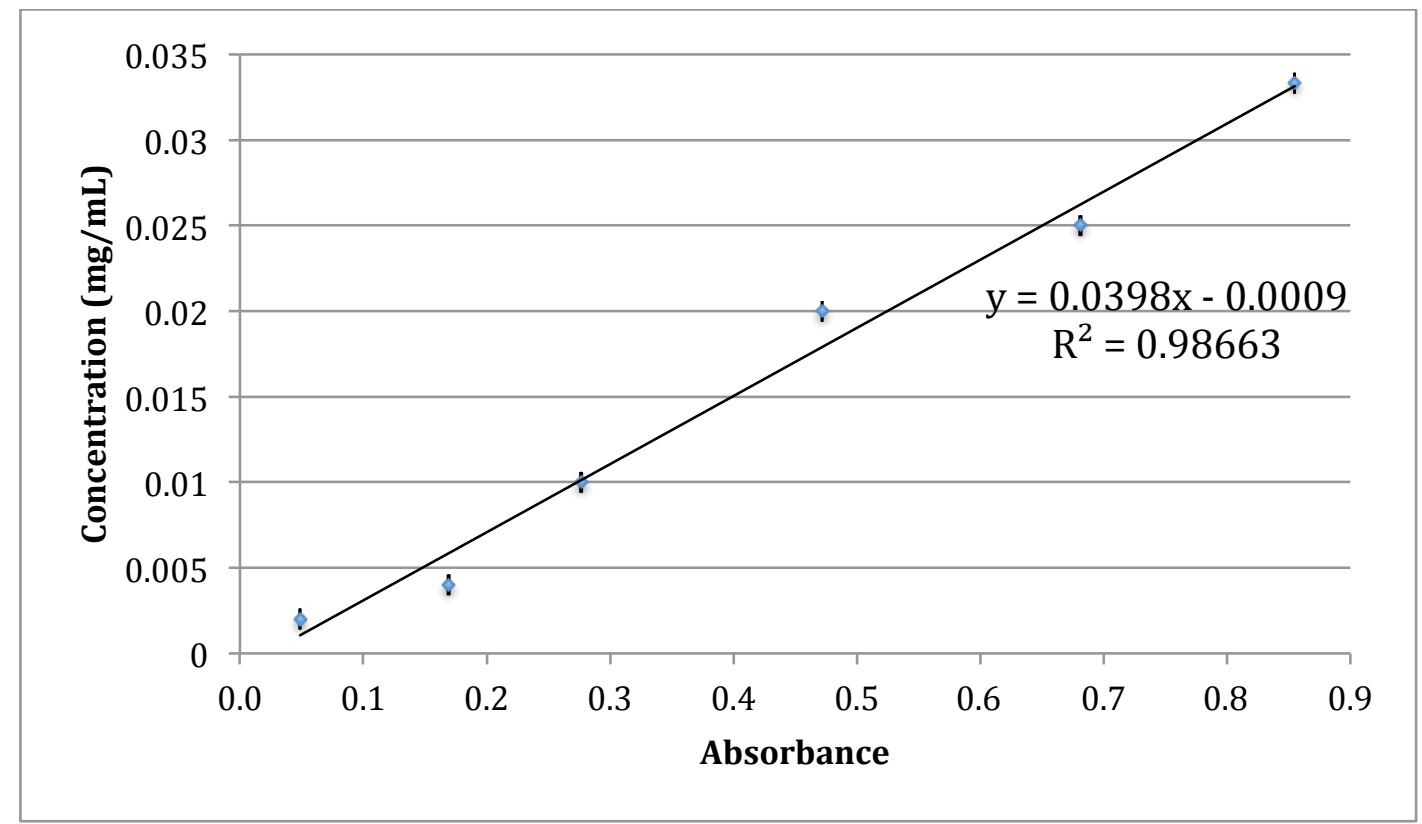

Figure 8. Tryptophan calibration curve of known concentrations versus absorbance taken at $280 \mathrm{~nm}$ in Tris buffer at $\mathrm{pH} 8.6$ using a $200 \mu \mathrm{L}$ quartz cuvette from Agilent Technologies. Error bars are extrema in 5 total absorbance readings. 


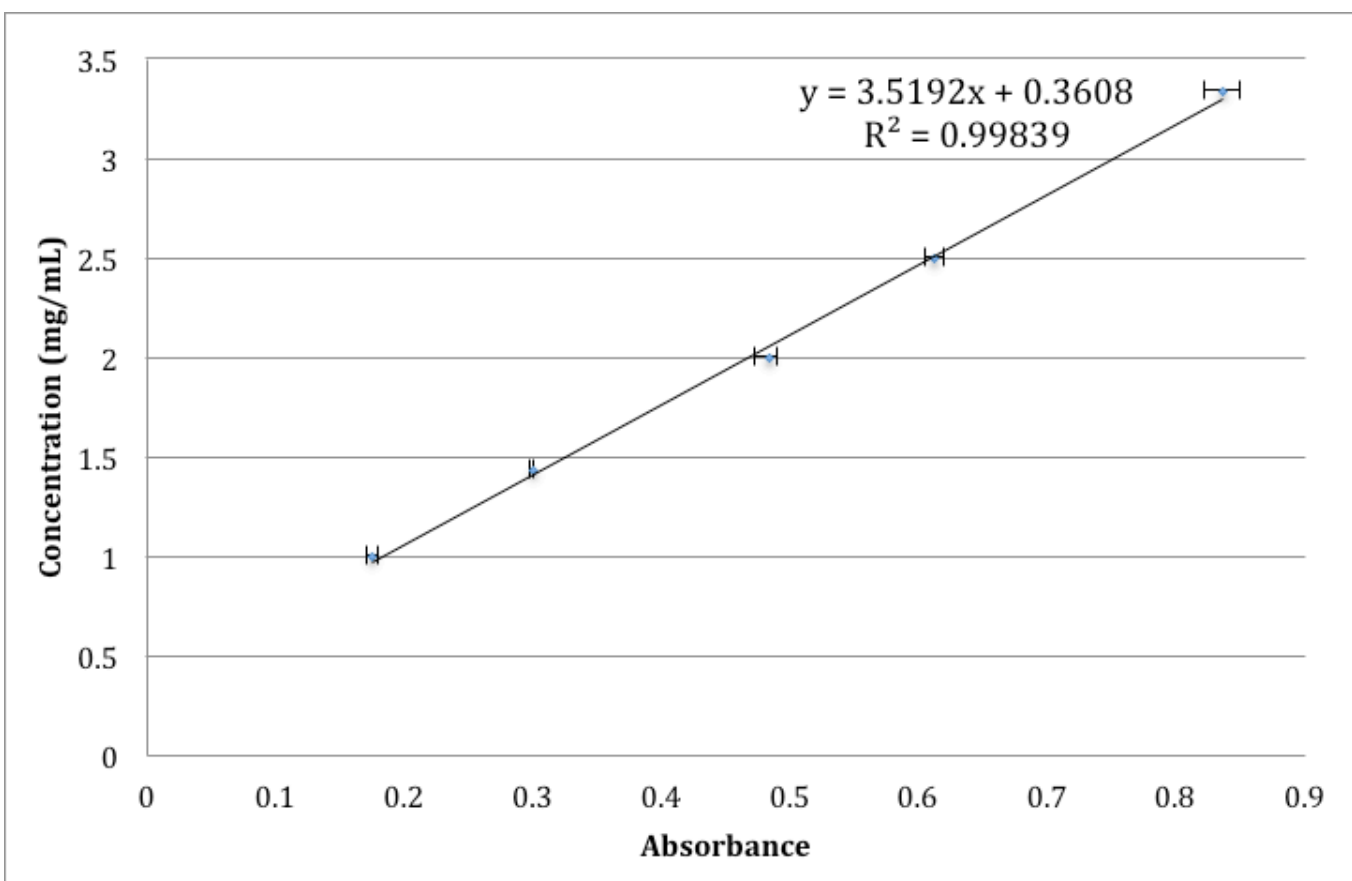

Figure 9. Glucose calibration curve of known concentrations versus absorbance taken at $500 \mathrm{~nm}$ in a plastic cuvette, after diluting 100-fold in Glucose Oxidase Reagent and warming at $37^{\circ} \mathrm{C}$ for 5 minutes. Error bars are extrema in 5 total absorbance readings.

The range of absorbance values that the calibration curves cover is wide. According to the Beer-Lambert Law, absorbance and concentration have a linear relationship only at absorbance values between 0.1 and 1.0. The range of Figure 8 spans absorbance below 0.1 and up to almost 0.9 , so the entire linear range is well covered. This means that no matter what the concentration of the unknown sample is, the calibration curve spans the entire linear range, which gives confidence at low and high concentrations. The lowest data point of Figure 9 is just lower than absorbance of 0.2 and shows that glucose is still linear up to absorbance of 0.9 . This range for the glucose concentration does not cover the very low end. For this reason, a very high concentration of glucose was used to incubate the alginate beads before diffusion to keep the data in the range of the calibration curve. 


\subsection{Bead Diameter Data and Discussion}

Figure 10 shows the average diameter of all the alginate bead samples measured after diffusion data were taken. The standard deviations for all bead diameters are less than $10 \%$ of the measured average, and for most groups is less than $5 \%$.

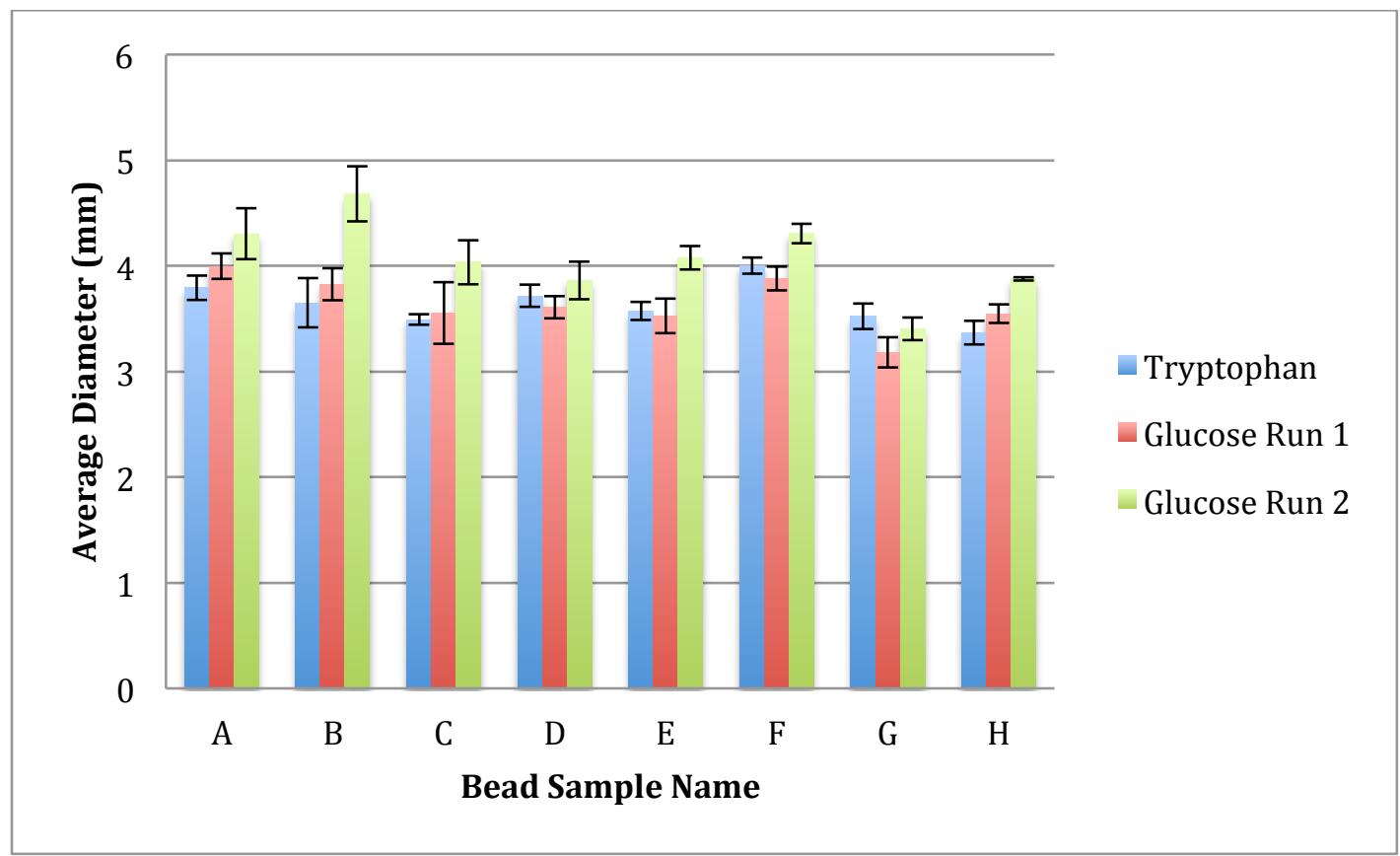

Figure 10. Average diameter for beads measured with a micrometer after diffusion trials for tryptophan and glucose runs. Error bars are the standard deviation from five measurements.

To determine if bead diameter stayed consistent between tryptophan and glucose runs for alginate beads made under the same conditions, all bead diameter measurements were plotted for each bead sample. The average from all measurements are shown in Figure 11. The calculated standard error in the full set of measurements is presented in Appendix A, Table 7. The standard error is less than $3.5 \%$ of the average measurement, which is acceptably low for all runs in the same bead sample. Therefore, the bead size is consistent between bead samples to allow comparison across different runs or solutes. 


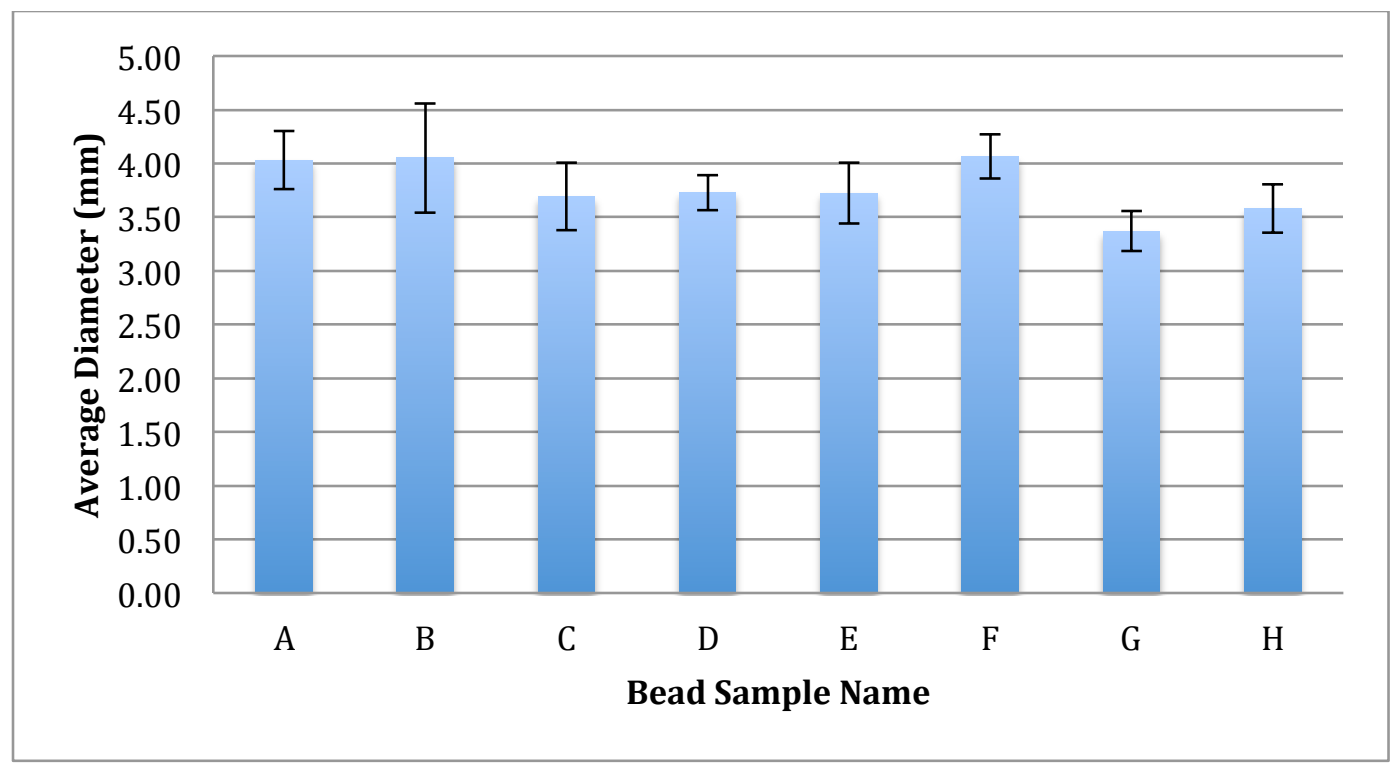

Figure 11. All measured bead diameters for all runs for each bead sample. Error bars are set as standard deviation of the set of measurements.

The data shows that making beads by adding alginate solution drop-wise from a syringe by hand created consistent sized spheres without need of more expensive setups (e.g. syringe pumps). The diameter compared for all runs showed a low standard error, which makes it reasonable to compare different runs with the same polymerization conditions. Visually, the beads looked spherical, with some spheres coming to a point. The shape seemed to be more consistently spherical when the distance between the syringe and the cross-linking buffer was kept constant. This distance needs to be far enough away to allow the droplet to form a sphere before it hits the cross-linking liquid. This exact distance was not studied, but could be important in future work [6]. It is possible to create different size spheres by using different syringe tips or smaller gauge needles, but specific polymerization conditions to achieve smaller diameter spheres were not studied in this work. There did not appear to be measurable swelling from the beads before and after the diffusion run. The overnight solution had a similar salt content to the 
solution used for the diffusion runs. This was done to reduce any possible swelling during the diffusion run. If swelling did occur in the alginate beads, it would have happened during this overnight incubation time, although it was not measured.

Therefore, it was assumed the radius stays constant during data collection, as the alginate beads would have already swelled in the overnight solution. Uniformly spherical shape and little to no swelling is critical for the math to be applied to the system.

\subsection{Diffusion Data and Fit to an Exponential}

Data from pilot tests to determine sample times are shown in Figures 12 and 13. The concentration over time was plotted to determine the time ranges with the highest flux through the spheres. The highest flux through the spheres would occur before the solute reaches equilibrium. Both tryptophan and glucose pilot tests were done using bead sample A. It is possible that the optimal time ranges for active diffusion could vary between different bead samples, but it was assumed that the variation between different bead samples would be smaller than minutes. Therefore, the sampling times used in the diffusion trials contained more data points in the first ten minutes and the same sampling times were used between all bead samples. From the data in the pilot tests, the first ten minutes covers the highest flux events of diffusion. 


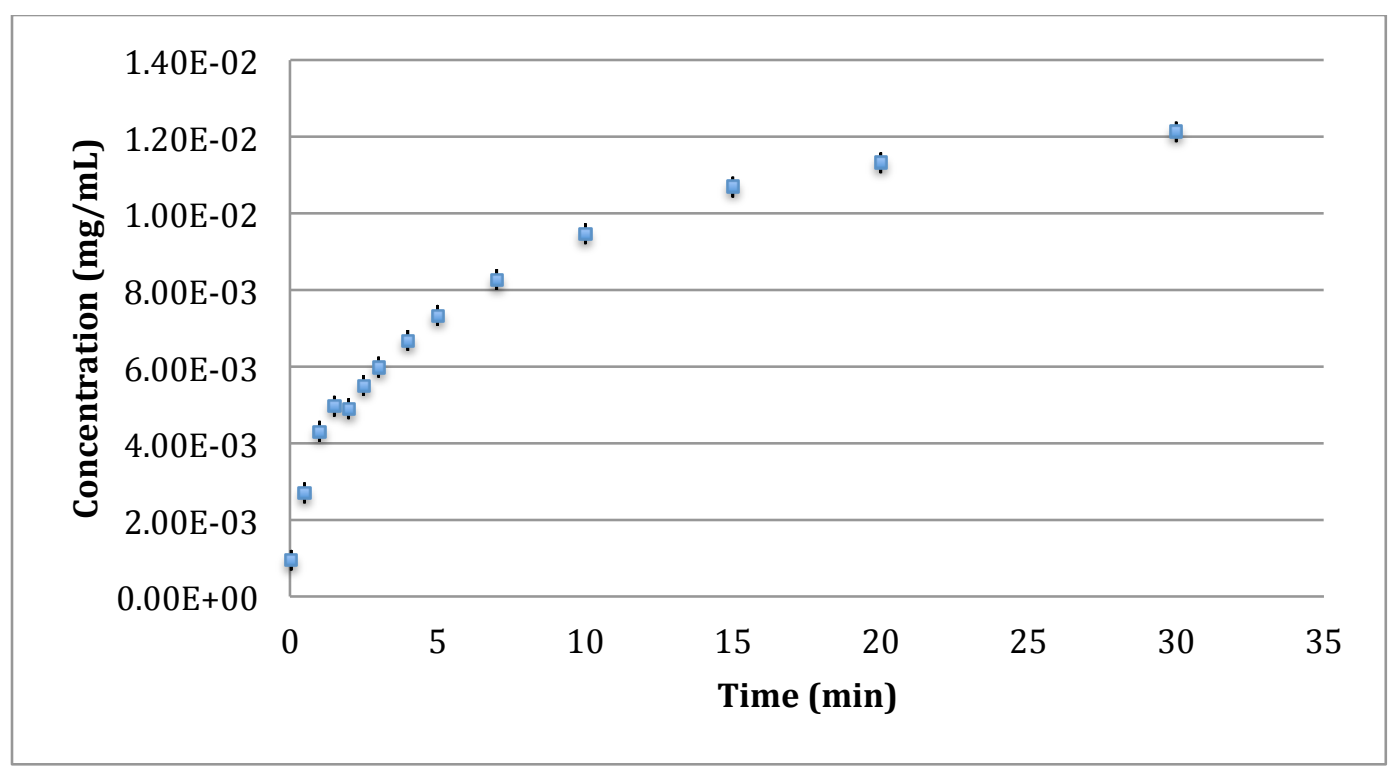

Figure 12. Tryptophan time test taken using bead sample A, measuring concentration of tryptophan released from the beads and into the bulk over time. Error bars are the standard deviation from five absorbance measurements using the spectrophotometer.

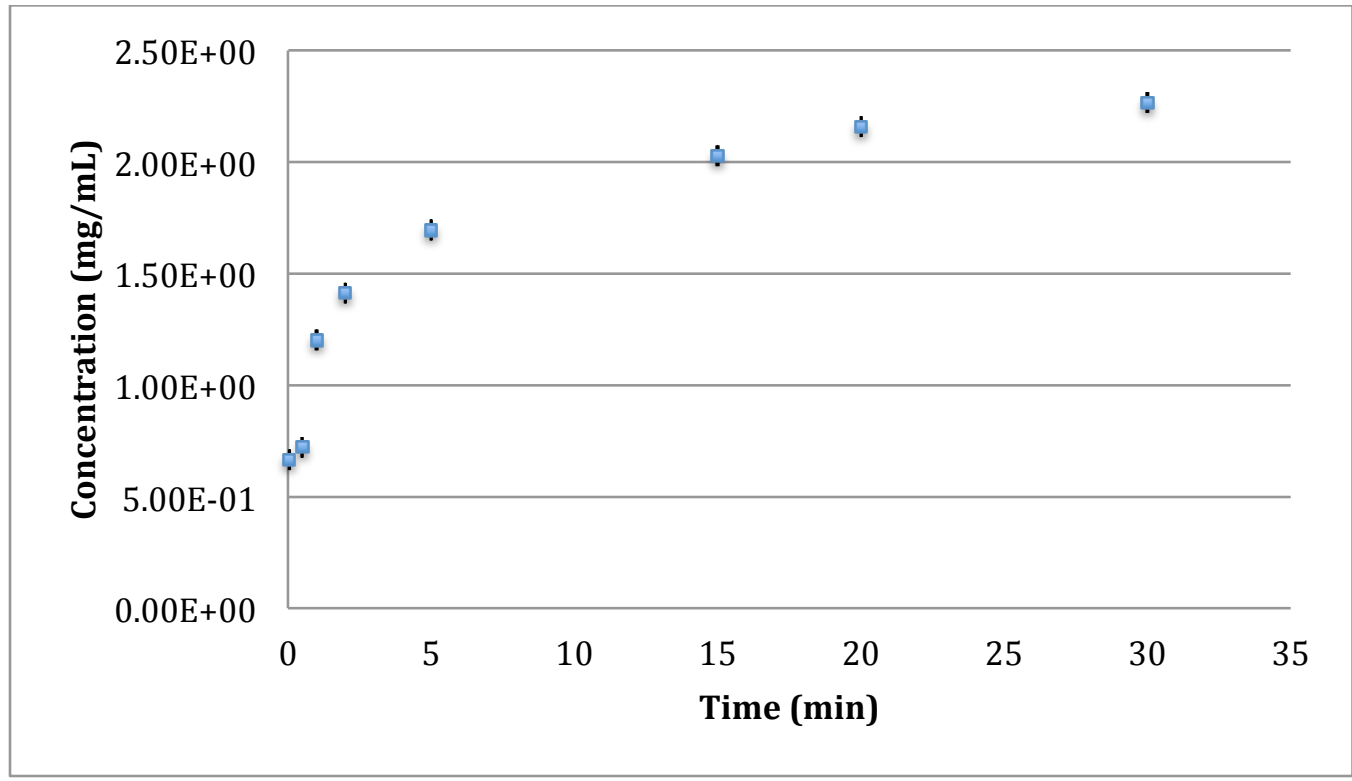

Figure 13. Glucose time test taken using bead sample A, measuring concentration of glucose released from the beads and into the bulk over time. Error bars are the standard deviation from five absorbance measurements using the spectrophotometer. 
The change in concentration over time was measured for glucose and tryptophan for all bead samples at the time points determined using the pilot test. To solve for the equilibrium concentration for each trial, Mathematica 10.2 was used to fit the data points to a general exponential function (Equation 10) using nonlinear regression. The limit as time goes to infinity gives the concentration at equilibrium, which is the asymptote of the function. This equilibrium concentration is needed to solve for diffusivity, using Equation 3. Figure 14 shows tryptophan concentration versus time for all bead samples and the fitted exponential functions. Fitted exponential equations and $\mathrm{R}^{2}$ from the nonlinear fit are displayed on each plot. The $\mathrm{R}^{2}$ for all tryptophan trials are above 0.99 , which means a close fit. Figures 15 and 16 shows both runs of glucose concentration versus time for all bead samples and the fitted exponential functions. The $\mathrm{R}^{2}$ for all glucose trials are above 0.9 , which indicates a good fit. 

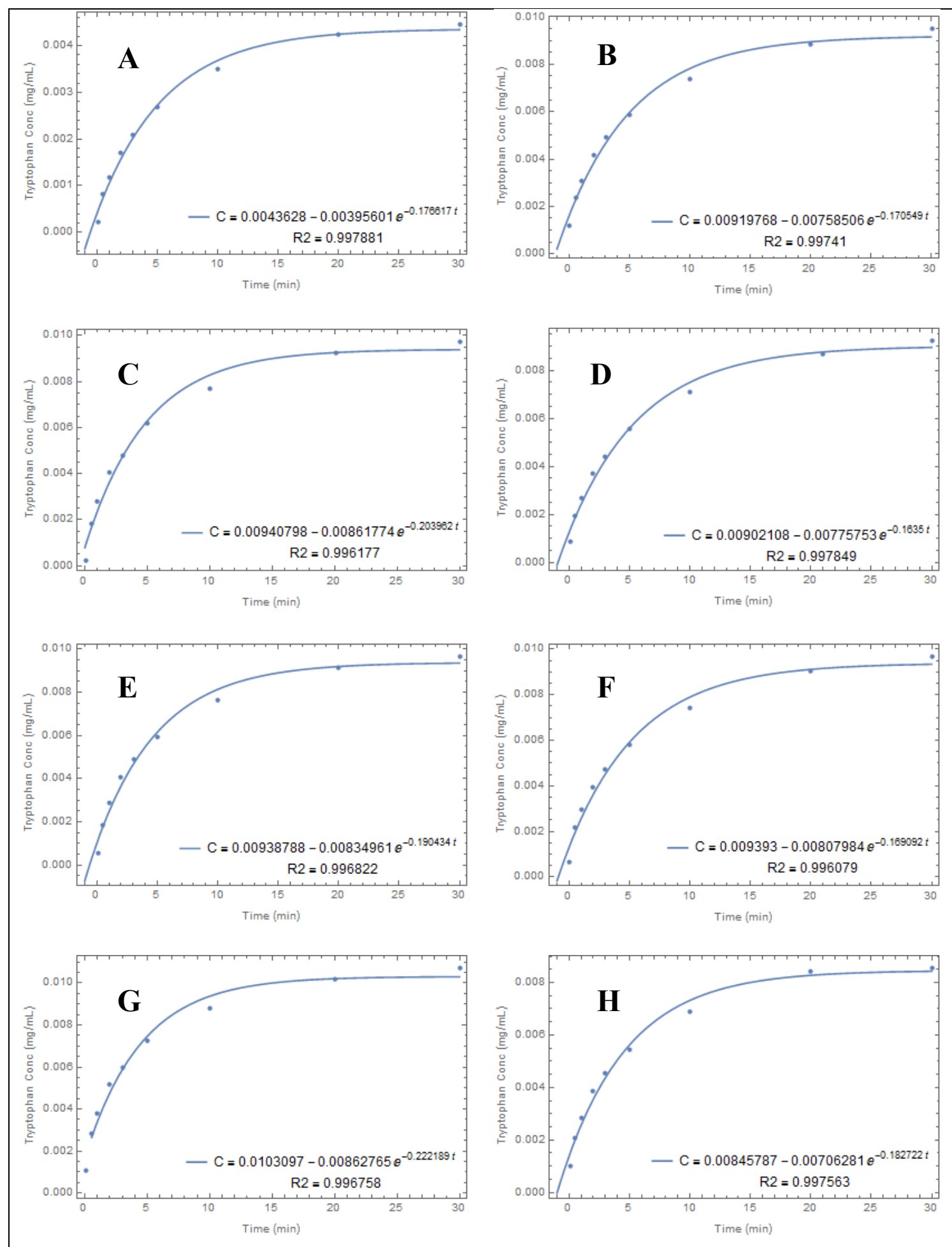

Figure 14. Diffusion data collected for all tryptophan trials, labeled with corresponding bead sample name, overlaid with exponential fit from Mathematica. 


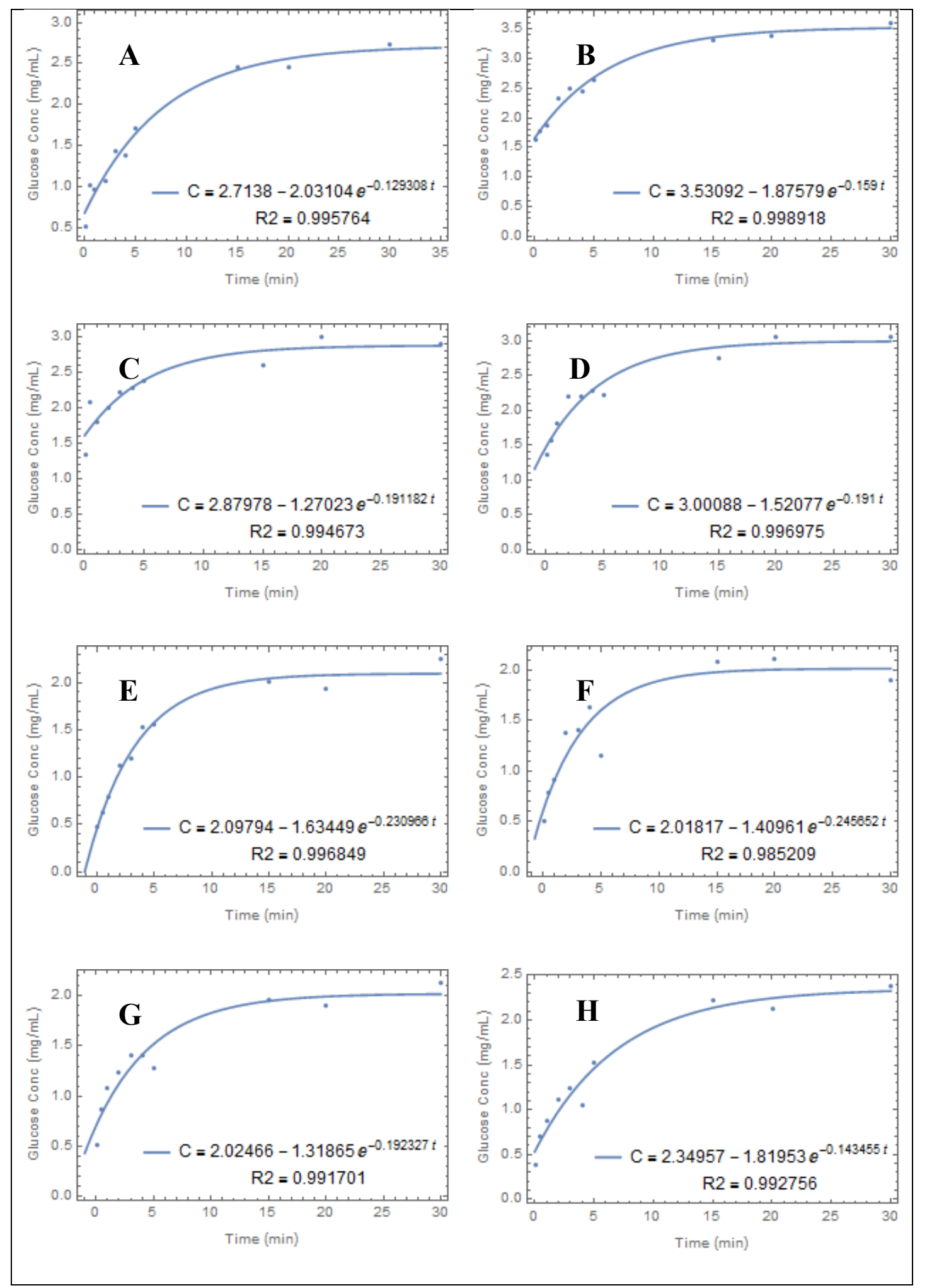

Figure 15. Diffusion data collected for the first glucose trial, labeled with corresponding bead sample name, overlaid with exponential fit from Mathematica. 


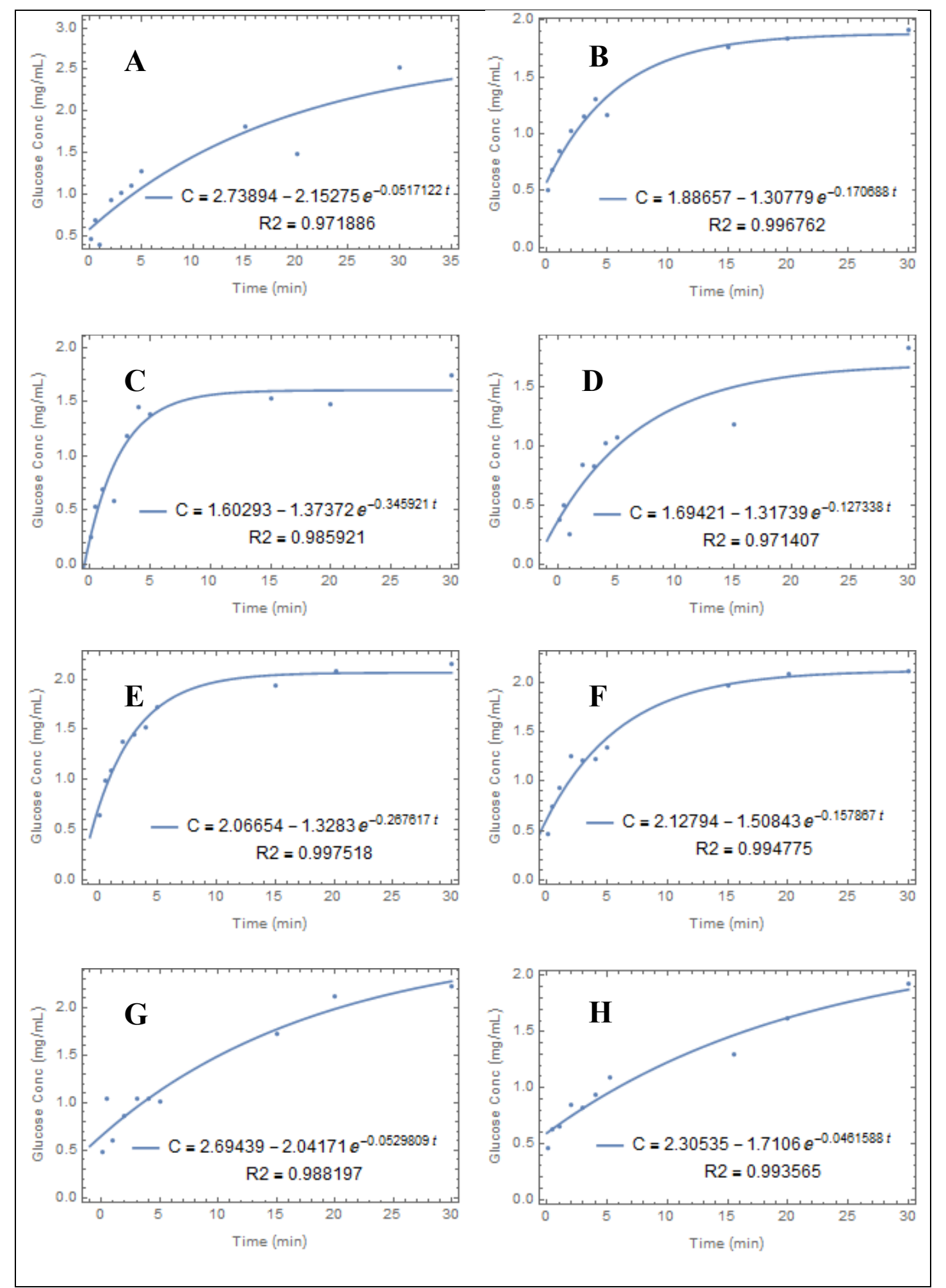

Figure 16. Diffusion data collected for the second glucose trial, labeled with corresponding bead sample name, overlaid with exponential fit from Mathematica. 
Between tryptophan and glucose, the calculated equilibrium concentration, $\mathrm{C}_{\infty}$, was higher for glucose, because a higher concentration of glucose is required to be detected by absorbance in the glucose oxidase reagent. It is interesting to note that in all diffusion trials, glucose has more noise in the data, which can be seen by looking at the residual plots for the nonlinear fits in Figures 14, 15, and 16. Residual plots for tryptophan bead samples are shown in Figure 17, and residual plots for both glucose runs are shown in Figures 18 and 19. The plotted residuals should be random if the model is a good fit. All residual results show data points randomly scattered about the origin. 


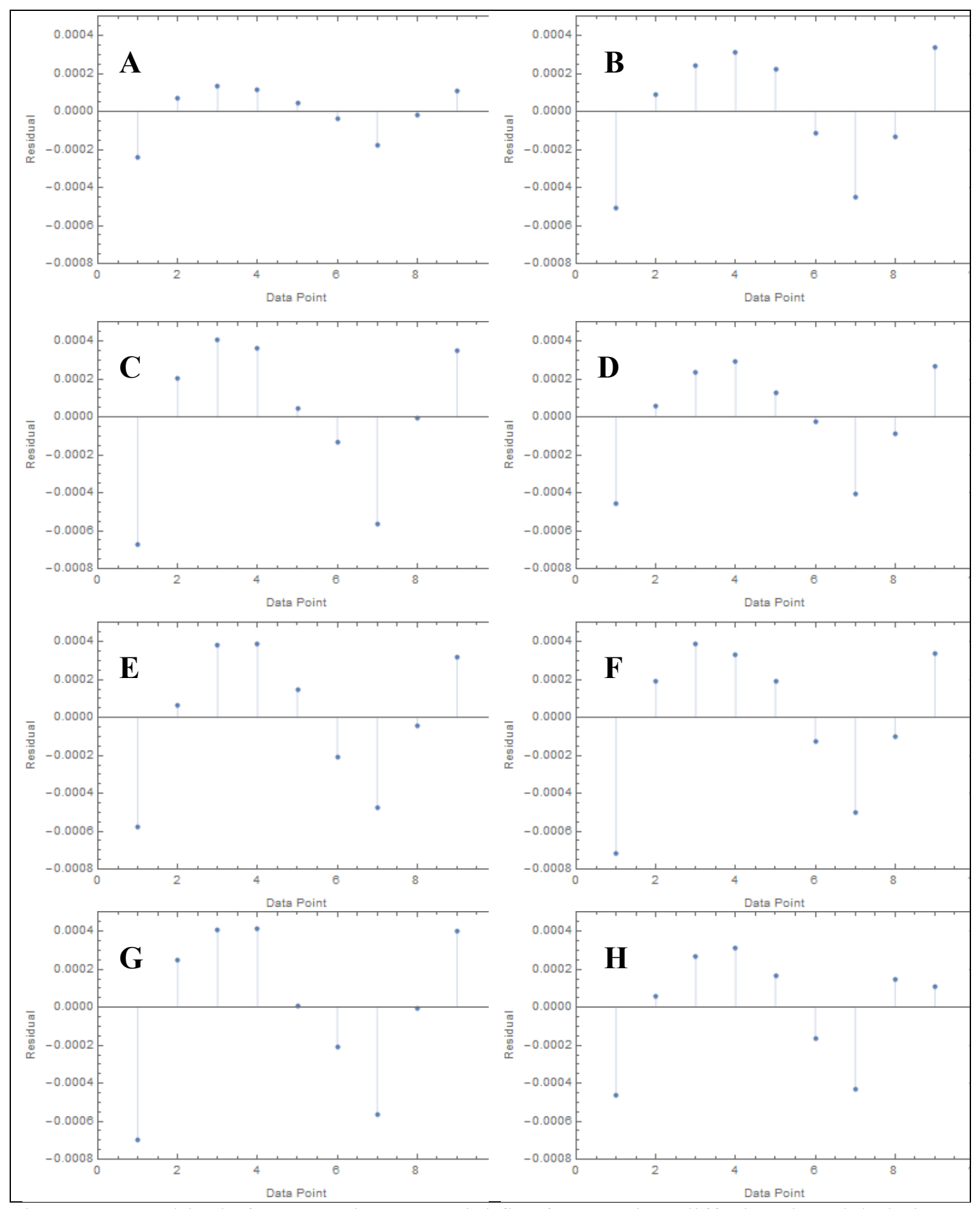

Figure 17. Residuals for general exponential fit of tryptophan diffusion data, labeled with corresponding bead sample name. The y-axis is fixed from -0.0008 to 0.0004 for all bead samples to show all data points. 

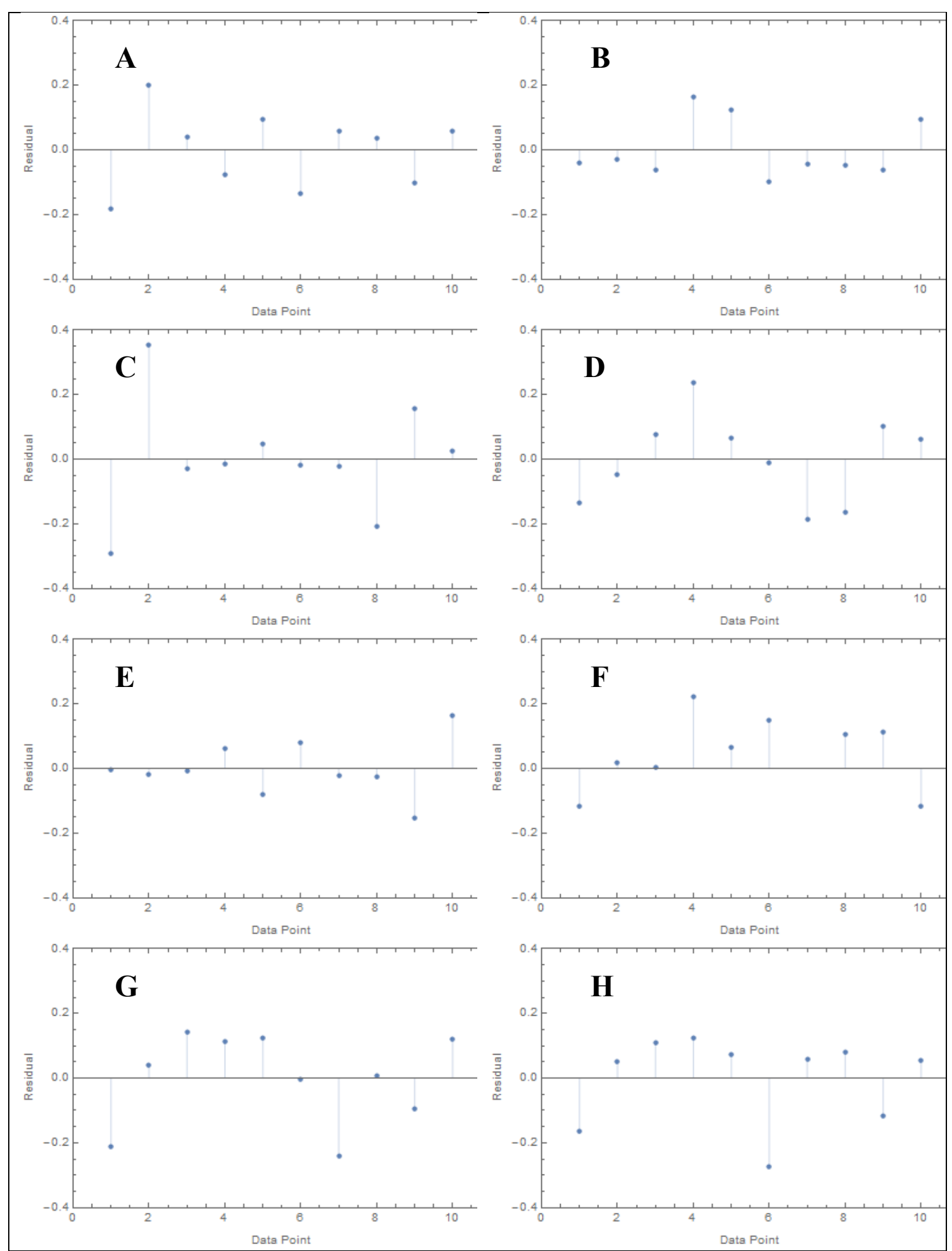

Figure 18. Residuals for general exponential fit of run 1 glucose diffusion data, labeled with corresponding bead sample name. The y-axis is fixed from -0.4 to 0.4 for all bead samples to show all data points. 

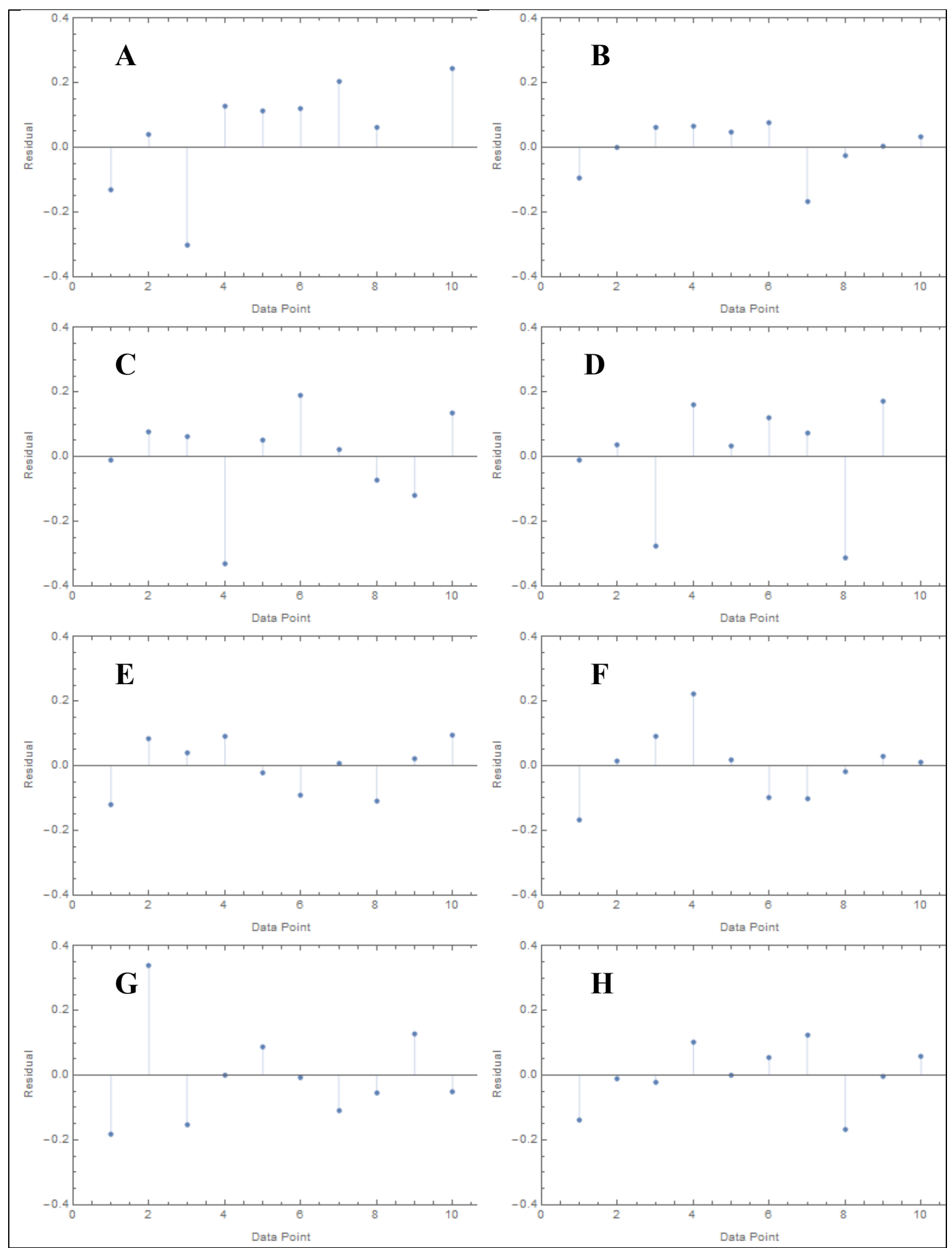

Figure 19. Residuals for general exponential fit of run 2 glucose diffusion data, labeled with corresponding bead sample name. The y-axis is fixed from -0.4 to 0.4 for all bead samples to show all data points. 
The plotted residuals in Figures 17, 18, and 19 show the difference in the noise between tryptophan and glucose when comparing the y-axis for the two solutes. Glucose residuals had to be plotted using a y-axis 1000-fold higher than the tryptophan residuals y-axis. This means there is a 1000 -fold difference in the variance between the measured data and theoretical model between glucose and tryptophan. Despite this noise, the high $\mathrm{R}^{2}$ and random residuals indicates that the nonlinear fit is acceptable for glucose. The increase in noise and variance in the glucose diffusion data compared to the tryptophan is likely due to using plastic disposable cuvettes to measure absorbance for glucose. Tryptophan samples were measured using an optically clear, washed and dried quartz cuvette to measure absorbance for each tryptophan sample. The plastic disposable cuvettes produce consistent data, but also introduce more noise due to scattering compared to quartz cuvettes. The two samples were also measured at different wavelength, so it is also possible that scattering at $500 \mathrm{~nm}$ is more pronounced in this system compared to scattering at $280 \mathrm{~nm}$.

\subsection{Diffusivity Calculation}

Diffusivity for all diffusion trials was calculated at every data point using Equation 3, where $C_{t}$ is the measured concentration for each time, t. Some diffusivity values were excluded from further analysis if they varied more than one standard deviation from the average of all calculated diffusivities, consistent with Chauvenet's Criterion [29]. The majority of excluded data points were at the early time points (e.g. time zero) and late time points, where equilibrium is almost reached (e.g. very little mass transfer occurring). This is expected at the early time points because the concentration of solute is very low 
and likely at or near the detection limit of the spectrophotometer. At the late time points, it is expected that some data points could be excluded because diffusion is driven by a gradient in concentration. At equilibrium with the bulk solution, the concentration gradient is zero, so diffusion is not driving solute out of the alginate beads.

Table 4 shows the consensus diffusivities and associated standard error for tryptophan and glucose runs compared to published literature values for diffusion in water. The literature values report the diffusivity of glucose and tryptophan in water [15] because comparison of diffusivity of these solutes to the exact alginate polymerization conditions is not practical or possible in this study. The majority of the calculated diffusivity values are the same order of magnitude of the literature values, and further statistical analysis will unravel the differences between bead samples for each solute.

Table 4. Average of calculated diffusivity for both tryptophan and glucose for all bead samples A through H compared to diffusion of the same solute in water. Reference data reprinted with permission from reference [15]. Copyright (1984) John Wiley and Sons.

\begin{tabular}{|c|c|c|c|c|c|c|c|c|c|c|}
\hline \multicolumn{2}{|r|}{$\begin{array}{l}\text { Polymerization } \\
\text { Conditions }\end{array}$} & A & B & C & D & $\mathbf{E}$ & $\mathbf{F}$ & G & H & Ref. \\
\hline \multicolumn{2}{|r|}{$\begin{array}{c}\text { Alginate } \\
\text { Concentration } \\
(\mathrm{w} / \mathrm{v} \%)\end{array}$} & 1.5 & 2.5 & 1.5 & 2.5 & 1.5 & 2.5 & 1.5 & 2.5 & \multirow{3}{*}{$\begin{array}{l}\text { Diff. } \\
\text { in } \\
\text { water }\end{array}$} \\
\hline \multicolumn{2}{|c|}{$\begin{array}{c}\mathrm{CaCl}_{2} \text { Concentration } \\
(\mathrm{w} / \mathrm{v} \%)\end{array}$} & 5 & 5 & 10 & 10 & 5 & 5 & 10 & 10 & \\
\hline \multicolumn{2}{|r|}{$\begin{array}{l}\text { Cross-link Time } \\
\text { (min) }\end{array}$} & 15 & 15 & 15 & 15 & 60 & 60 & 60 & 60 & \\
\hline \multirow{2}{*}{ 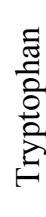 } & $\begin{array}{c}\text { Average } \\
\text { Diffusivity } \\
\left(\mathrm{x} 10^{-4}\right)\left(\mathrm{cm}^{2} / \mathrm{min}\right) \\
\end{array}$ & 9.45 & 12.52 & 7.67 & 4.57 & 15.2 & 5.71 & 5.22 & 5.26 & \multirow[t]{2}{*}{4.02} \\
\hline & $\begin{array}{l}\text { Standard Error } \\
\left(\mathrm{x} 10^{-4}\right)\end{array}$ & 6.82 & 9.06 & 3.27 & 1.50 & 10.2 & 1.47 & 1.53 & 1.41 & \\
\hline \multirow{2}{*}{ 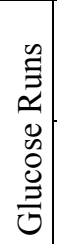 } & $\begin{array}{c}\text { Average } \\
\text { Diffusivity } \\
\left(\times 10^{-4}\right)\left(\mathrm{cm}^{2} / \mathrm{min}\right)\end{array}$ & 4.17 & 7.05 & 7.73 & 5.91 & 3.97 & 7.03 & 4.01 & 3.45 & \multirow{2}{*}{4.08} \\
\hline & $\begin{array}{l}\text { Standard Error } \\
\quad\left(\mathrm{x} 10^{-4}\right)\end{array}$ & 1.09 & 1.90 & 2.34 & 1.15 & 1.13 & 1.87 & 0.92 & 1.12 & \\
\hline
\end{tabular}


More repeated runs for both solutes would have added more stability and confidence to the analysis presented here, especially given the diffusivity through the membranes were often higher than the diffusivity reported in water. At most the diffusivity should be equal to the diffusivity in water, or lower due to the mass transfer being impeded through the membrane's pores. The measured data does not agree with theory, likely due to the small data set. The experiment was planned to provide an outline of an experimental setup and mathematical analysis for future students. Due to time constraints, repeated runs were not performed for tryptophan and only one repeat was performed for glucose. The relative differences between the different bead samples can be analyzed.

\subsection{Sources of Error}

There are many sources of error in an experimental setup of this type. The errors could be better quantified if more repeated runs were performed. Pipette error should be consistently random and relatively small, as the pipettes used for liquid handling were checked each day of use to confirm the calibration was acceptable. Volumetric measures for making stock solutions and initial water or buffer addition for each diffusion trial were taken using appropriately sized pipettes or graduated cylinders to keep volume measurement error low.

Incomplete mixing would cause concentration gradients around the alginate beads. To address this, a stir plate was used for all diffusion trials and the same stir speed was used for every run. If stirring is sufficient, the concentration boundary layer along the outside of the spheres would be smaller, and in the equations in this experiment the thickness is assumed to be zero. If stirring is insufficient, there would be a concentration 
boundary layer on the surface around each sphere, and sampling the bulk would not be an appropriate way to model the diffusion with Equation 3. There was no study done to determine if the mixing speed is sufficient to reduce the boundary layer on the alginate spheres. Therefore, the concentration in the bulk was assumed to be equal to the concentration on the surface of the spheres as described in Crank's development of Equation 3.

It has also been shown in the literature [23-25] that swelling can occur when changing the solution in which the beads are stored. If the beads swell during data collection, the radius cannot be assumed constant, and the mathematical equations from Crank [18] are no longer valid. By incubating the beads overnight in the same buffer used for data collection, this error should be minimal in this experimental setup. Additionally, the ratio of alginate beads to bulk solution is an important variable. If the ratio is too high, there may not be enough fluid in the bulk to allow for samples to be removed and still assume a constant volume. If the ratio is too low, the diffusion rate out of the alginate beads may be too small to be observed due to the dilution ratio being too large. In this experiment, a ratio of 1:10 was used which allowed enough fluid to assume a constant volume and a measurable concentration increase over time.

For making the alginate beads, the largest source of error is inconsistent drop size due to human. A syringe pump would make more consistently sized spheres, as pressure on syringe would remain constant. To quantify this error, the bead diameter was measured and discussed in Figure 10, which showed the variation in bead size to be acceptably small. There is also human error in measuring time by using cross-link time as a 
variable. Time measurement was also important when measuring diffusion data. For all data points, the time the sample was taken in reality was used instead of the time planned. For example, if a sample was actually removed from the bulk at 20 minutes and 5 seconds instead of the planned 20 minutes, then the time used for that data point was 20 minutes and 5 seconds. Within two to three seconds of error is expected from this method, which is sufficiently small.

Variations in the spectrophotometer measurements can cause error. The same spectrophotometer was used throughout the entire experiment, which was blanked before each diffusion trial began. To quantify the error from the instrument, all absorbance values were measured five times on the instrument and the standard deviation was calculated from the five replicated measurements. For every data point, the ratio of standard deviation to the average of the five measurements was always less than $10 \%$ and usually less than $2 \%$. For the samples taken at the very beginning of a diffusion trial ( 3 seconds after adding the solute-free solution), there is larger variation in the repeated instrument readings. These data points are all very close to zero absorbance, so there is more noise in the data. This phenomenon can be visualized in Figures 20 and 21 for tryptophan and glucose, respectively. The time of sampling versus the ratio of standard deviation to average is plotted. The highest variations are always at the time zero point, which was usually taken 3-5 seconds after the solute-free solution was added. The zero point also always had the lowest absorbance value, so comparing standard deviation at this data point does not show how small this variation is normalized to the entire range of absorbance values across the diffusion trial. Even though the variation is high in the 
early time points, the value, when converted to concentration using the calibration curves, gives a very low concentration of solute. Therefore, variation from the early time points is sufficiently small and less impactful and the variation in the later time points is shown to be below $2 \%$ for most bead samples.

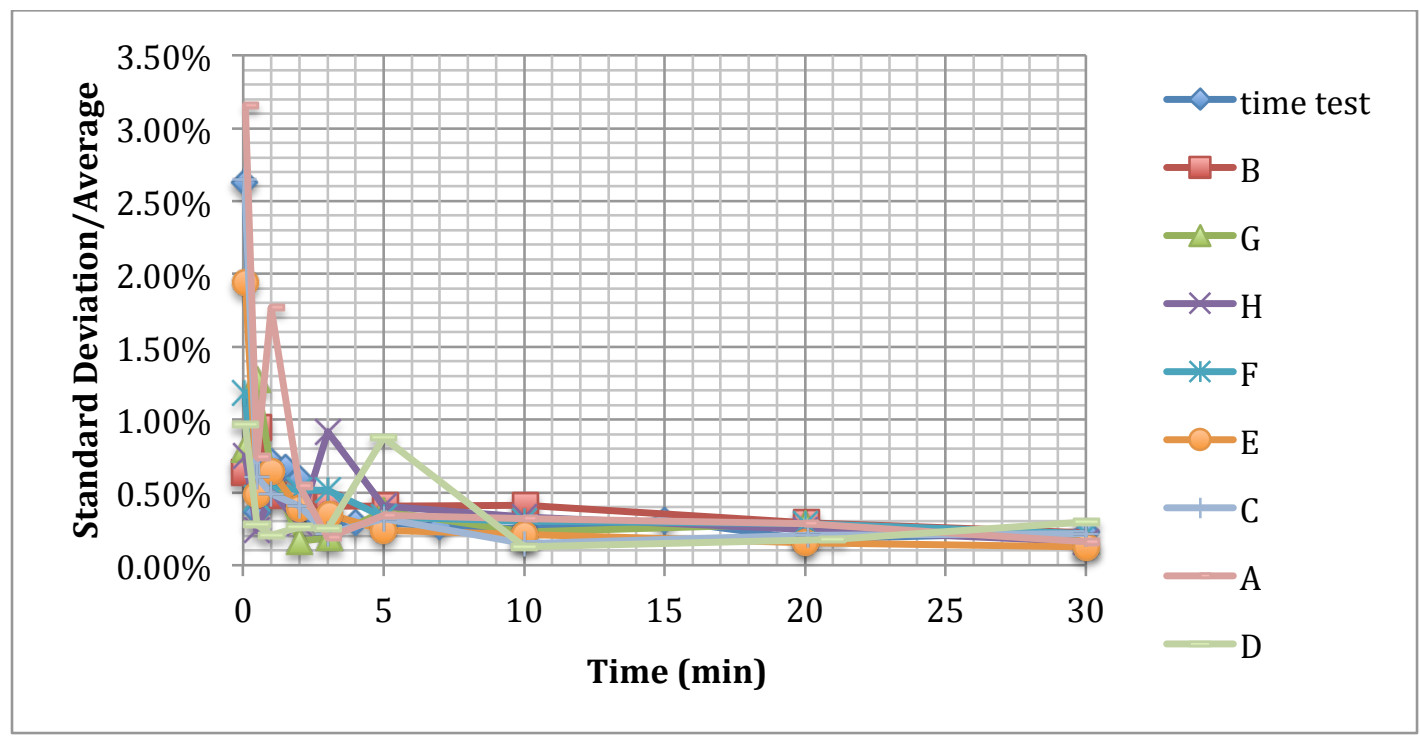

Figure 20. Ratio of standard deviation and the average of five absorbance values plotted versus time for all tryptophan data points.

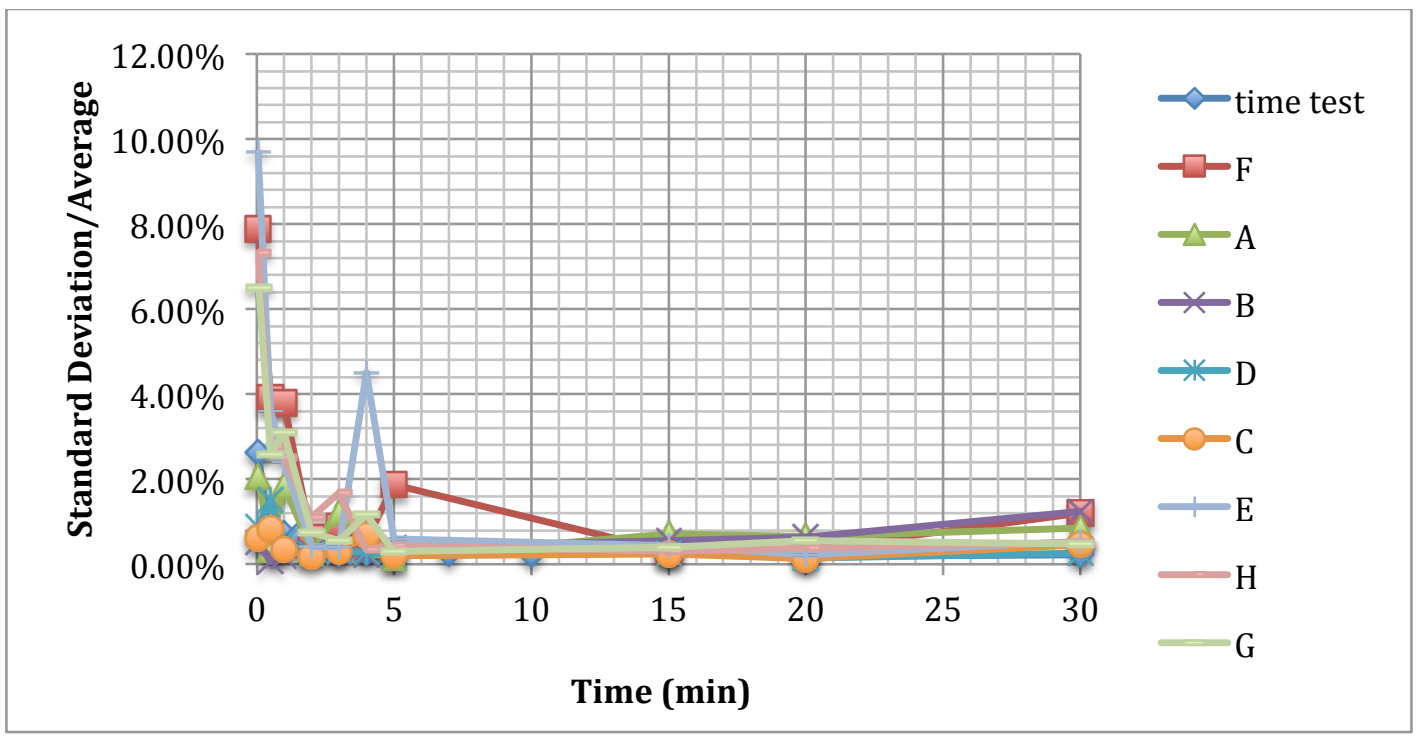

Figure 21. Ratio of standard deviation and the average of five absorbance values plotted versus time for all glucose data points. 
Glucose itself does not absorb light to be measured on a spectrophotometer, so the glucose oxidase reagent is used to produce a colored product that absorbs in the visible spectrum. Diluting the glucose sample with the oxidase reagent, then incubating for five minutes at $37{ }^{\circ} \mathrm{C}$ adds variation from pipetting, timing, and controlling temperature. After producing the colored product, the sample was measured in a plastic disposable cuvette suitable for the visible spectrum, which has higher scattering. This introduces more noise in the absorbance measurements. The noise from all these sources in the glucose was controlled as best as possible by using a water bath to control temperature, for instance, instead of a less reliable heating method. The nonlinear fits Figures 15 and 16 still gave good fit reports, so the variations from these sources were randomly distributed across all data points. The residual plots in Figures 18 and 19 confirm this finding for glucose runs.

Another source of variation is from assumptions made in the calculation and compiling all calculated diffusivity values to be analyzed by DOE. To calculate diffusivity, Equation 3 was used to solve for $\mathrm{D}$ for each concentration and time data point. In order to analyze diffusivity across all bead samples, a consensus diffusivity was determined for each bead sample. Chauvenet's Criterion was used to exclude diffusivity values that varied too far from the average. This was done to increase confidence of the final diffusivity value used for comparative analysis. Excluding errant data points tightens the variation between samples, so the average diffusivity of all included points better represented the overall trend in the data. These consensus diffusivities were used for all further DOE calculations to compare relative differences and calculate main 
effects in the experiment. All data points were subject to the same exclusion criterion, so the variation should be randomly distributed.

\subsection{Diffusivity Results}

A full-factorial analysis was performed to determine the main factors and interactions affecting the responses of diffusivity and initial concentration, $\mathrm{C}_{0}$. Figure 22 shows the effects of all factors and interactions on the calculated diffusivity for glucose and tryptophan calculated by using Equation 9. The magnitude of the columns in Figure 22 signifies a more influential effect on the diffusivity response. Factors or interactions with a positive effect on diffusivity resulted in a higher diffusivity when the level for that factor was high. For example, alginate concentration shows a positive effect on tryptophan diffusivity in Figure 22, because the average of all diffusivities at 2.5\% alginate is higher than the average of all diffusivities at $1.5 \%$ alginate.

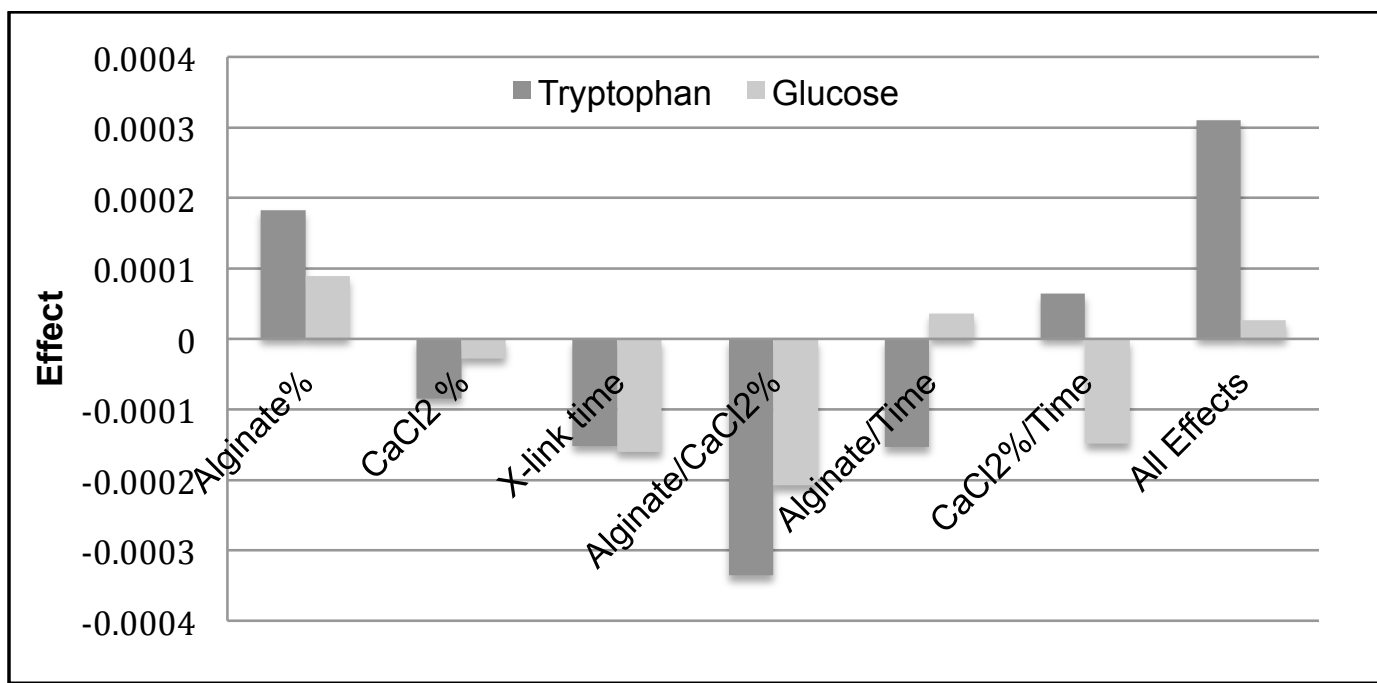

Figure 22. Effects and interactions on diffusivity of tryptophan and glucose. 
Another way to visualize the calculated effects on diffusivity is by using a line to plot effects of each source and observe the magnitude and sign of the slope. In JMP, this is called a prediction profiler. This tool can be useful to visualize the data in a different way, but does not show interactions between factors. The prediction profiles for tryptophan and glucose are presented in Figures 23 and 24. Alginate concentration showed a shallow but positive effect for both tryptophan and glucose. Cross-link concentration showed an almost neutral slope for both solutes. Cross-link time showed a negative slope for both tryptophan and glucose.

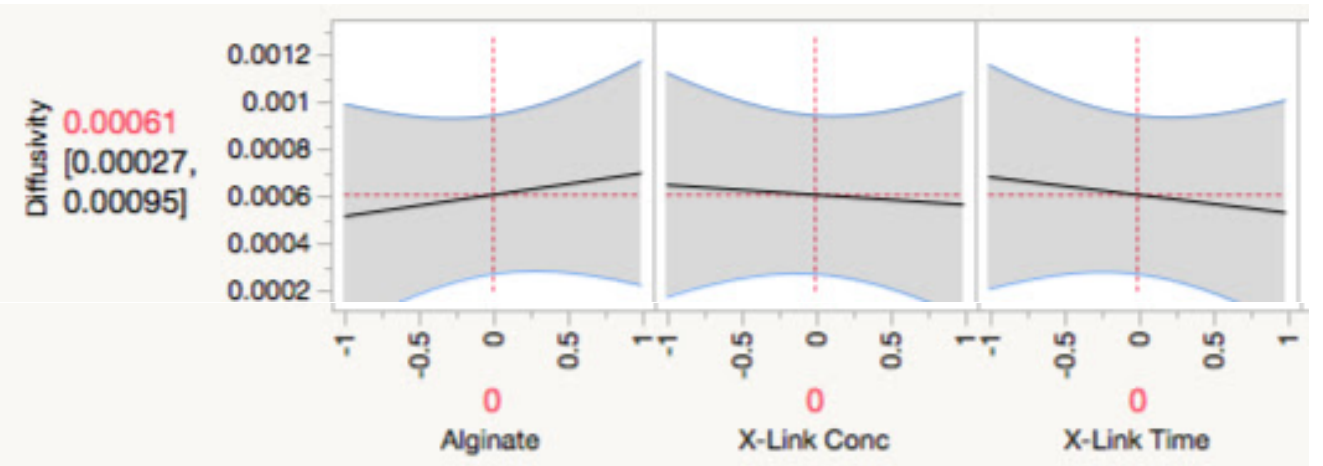

Figure 23. Prediction profile for tryptophan diffusivity created using JMP. 


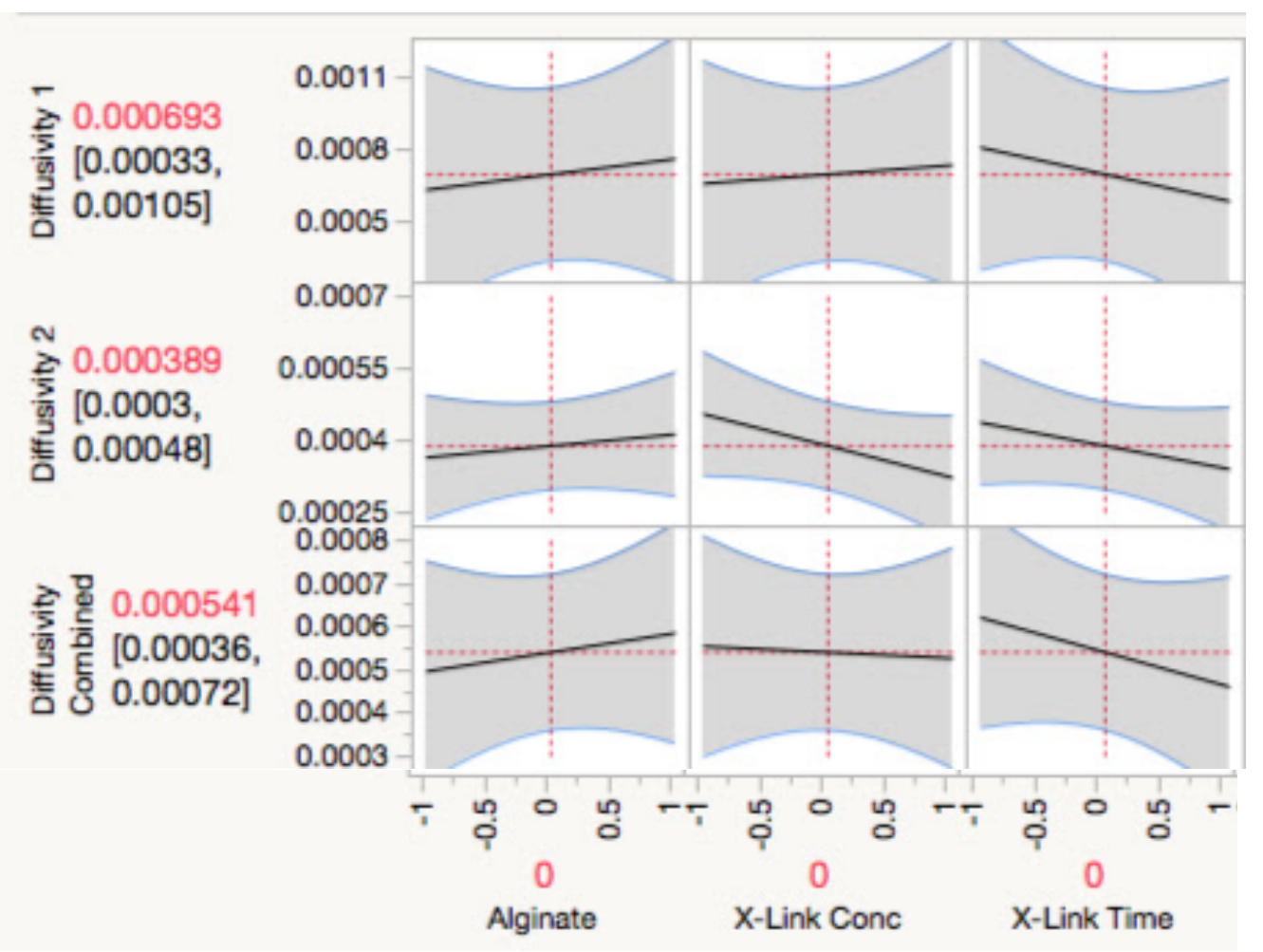

Figure 24. Prediction profile for glucose diffusivity created using JMP.

The calculated effects presented in Figures 22, 23, and 24 cannot show which effects and interactions are statistically significant. Further analysis is needed to show main effects with statistical confidence. A half-normal probability plot was generated for tryptophan diffusivity, in Figure 25, and glucose diffusivity in Figure 26, to show which effects vary outside of normal error. Effects and interactions that fall on the line vary due to normal error. Effects and interactions that are outside the line are likely significant, as they are not due to normal error. 


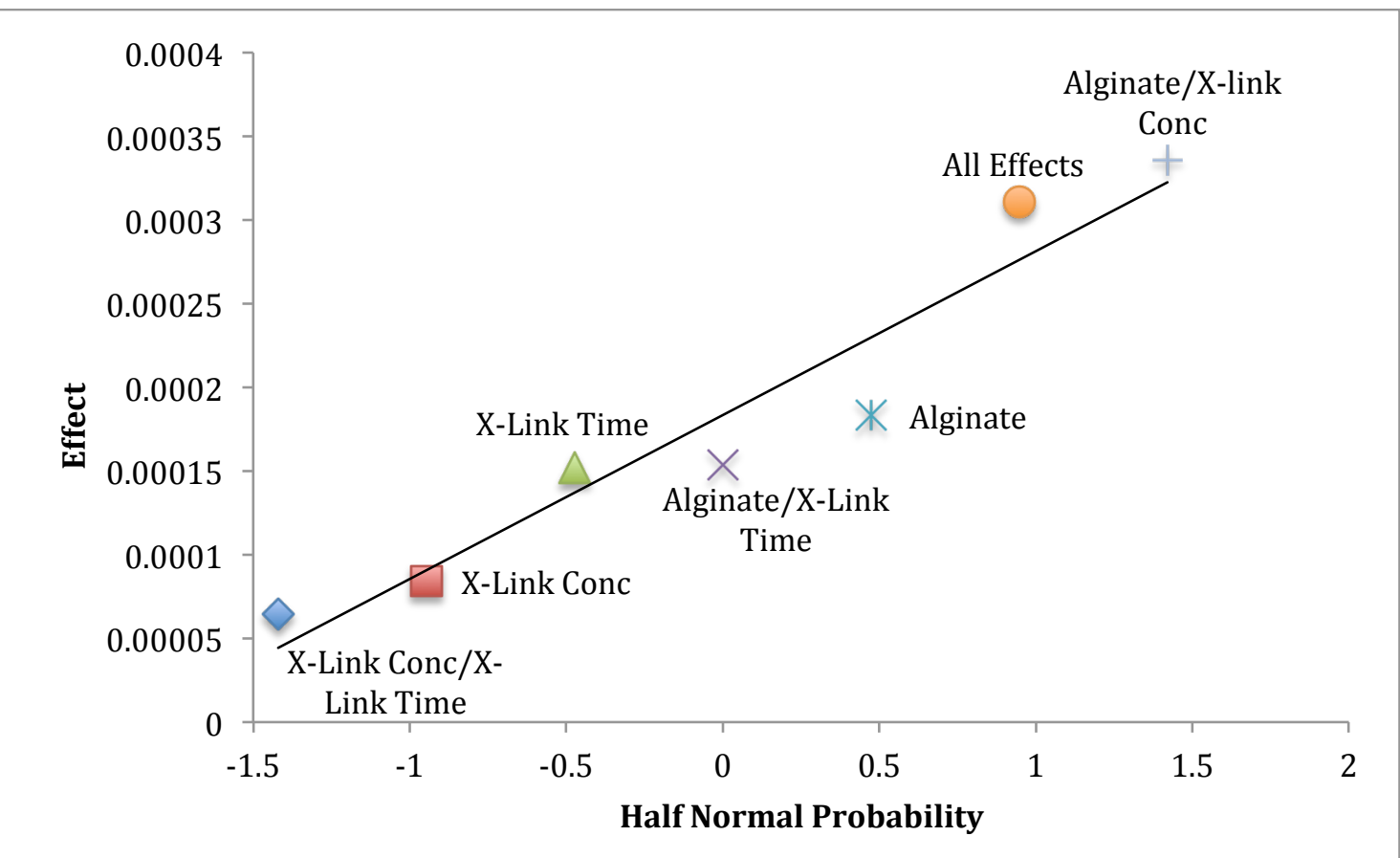

Figure 25. Half-normal probability plot of tryptophan diffusivity effects and interactions.

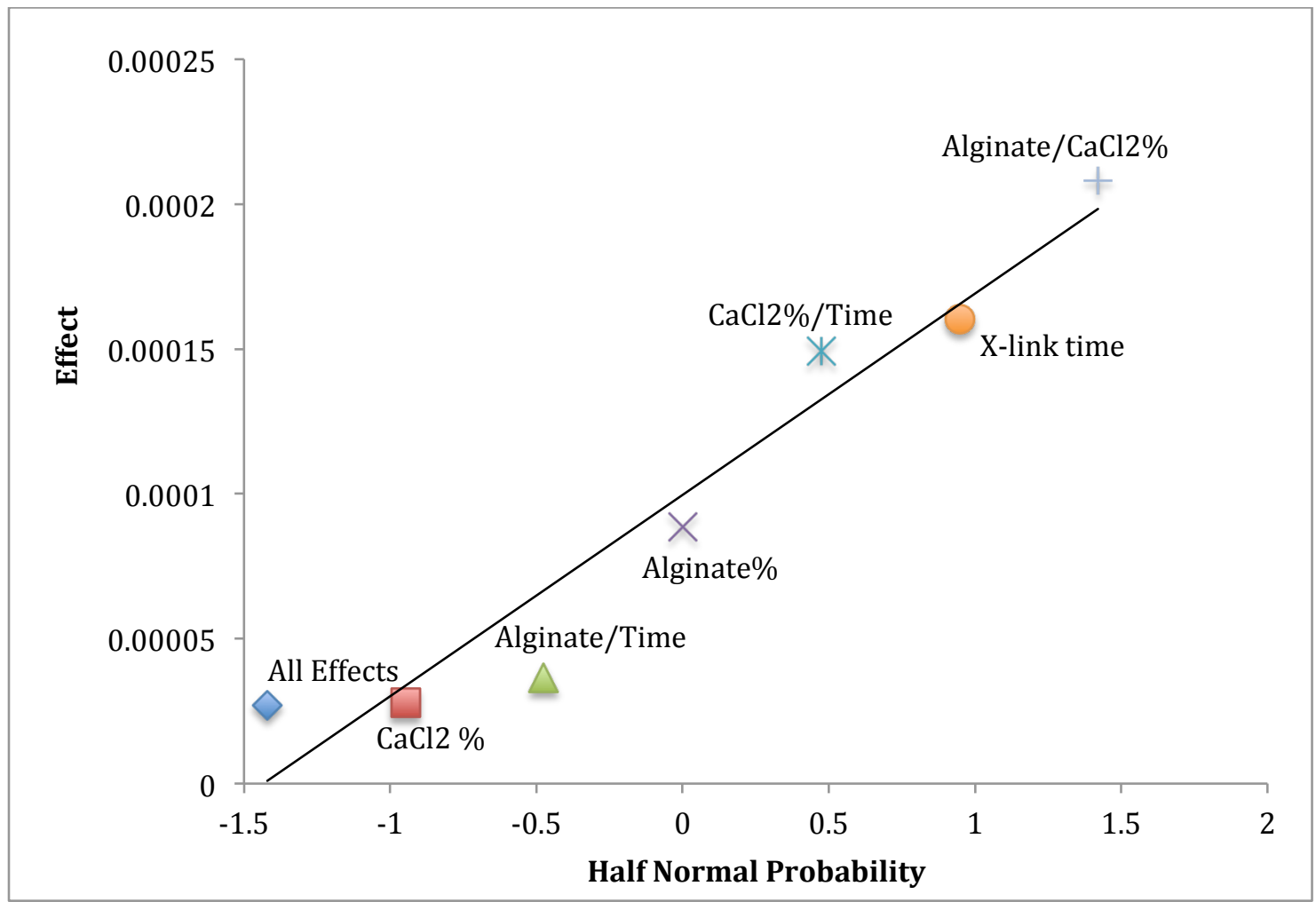

Figure 26. Half-normal probability plot of glucose diffusivity effects and interactions. 
Both half-normal probability plots show that all factors and interactions fall on a line, therefore no factor or interactions influence the diffusivity of tryptophan or glucose outside of normal error. In other words, the polymerization conditions tested in this work had no measurable effect on the calculated diffusivity. Half-normal probability plots are a useful visualization tool, but not quantitative. The statistical impact of this and all factors and interactions can be verified by ANOVA.

To verify that ANOVA is applicable, the assumptions of ANOVA, outlined in Verma's book [11], need to be checked. First, the observations need to be independent. In this experiment, the observations are the concentration over time, and this data was independently taken for each bead sample. In fact, new bead samples were generated for the repeated run with glucose. Therefore, this assumption is acceptable, since all diffusion trials were done individually. Second, ANOVA assumes that residuals be randomly distributed, which was addressed by the discussion of Figures 17, 18, and 19. Third, all variances between groups are the same. In this case, the term "groups" mean the source of variation, which would be a factor such as alginate concentration. The discussion in Section 6.6 on sources of error presents the many of the sources of variation in this experiment. These variations are equally distributed across all diffusion trials. Therefore, all assumptions for ANOVA look reasonably met to apply ANOVA to the data.

ANOVA tables were generated using calculations from JMP, and are presented in Appendix A, Tables 8-11. The Source columns specify the source of variation, which is the fitted model, a factor, or interaction. JMP also calculates the sum of squares, or the 
total variation explained by each source. The sum of squares is used to determine the $\mathrm{F}$ ratio. The $\mathrm{F}$ ratio is calculated by dividing the source mean square by the error mean square. If the $\mathrm{F}$ value is high, then the source varies further from the model prediction, and could be a main effect in influencing the response. The column titled "Prob $>$ F", henceforth called the $\mathrm{P}$ value, is the probability of obtaining the $\mathrm{F}$ value of each source. In general, a $\mathrm{P}$ value of 0.05 or less is considered significant, which means the source would have a $5 \%$ or less probability of having that variation due to normal error. Therefore, it is likely that the variation is due to the source's contribution to the response. In other words, that factor or interaction would be considered a main effect.

The ANOVA confirms the same conclusion drawn from the half-normal plots in Figures 25 and 26, which is that no factors or interactions influence tryptophan or glucose diffusivity outside of normal error. None of the $\mathrm{P}$ values are lower than 0.05 , so none of the factors or interactions are main effects on diffusivity. Although traditionally a $\mathrm{P}$ value lower than 0.05 is the threshold for significance, there are two sources for glucose diffusivity that are just under $11 \%$ probability: cross-link time and alginate by cross-link concentration. For subsequent discussion of results, these two effects will be regarded as main factors influencing glucose diffusivity. These two sources do not show variation off the line on the half normal plot (Figure 26), but ANOVA is a more statistically robust method than half-normal probability plots, which are credited as being qualitative.

JMP uses a prediction model to fit the variation of each source. The fit report statistics of the prediction model obtained from JMP are shown for tryptophan and glucose, respectively, in Appendix A, Tables 12-13. The R-squared, adjusted R-squared, 
root mean square error, mean of response, and the number of observations used are reported. The R-squared is the ratio of the sum of squares of the model versus the corrected total. An R-squared of 1 would mean that the prediction model fit the responses perfectly with no variation from error. The fit reports also show the adjusted Rsquared, which normalizes R-squared by the degrees of freedom to make the fit model comparable to systems with different number of variables or responses. This value is not needed for this analysis since the experiments between tryptophan and glucose had the same degrees of freedom, so the R-squared itself is comparable between the two. Root Mean Square Error refers to the standard deviation of the assumed random error. Mean of Response is the arithmetic mean of all response values, in this case, the diffusivity values for the solutes. The Observations refers to the number of trials that were fit by the prediction model, which were all 8 bead samples for both tryptophan and glucose.

The fit report for tryptophan diffusivity shows that the fit of the prediction model accounts for 0.679 or $67.9 \%$ of the diffusivity responses, leaving $32.1 \%$ as variation from error. For glucose, the fit report shows that $99.4 \%$ of the responses fit the prediction model, which leaves only $0.6 \%$ as variation due to error. For a good fit, an R-squared should have a value of 0.9 or higher and the Root Mean Square Error should be small compared to the Mean of Response. The Root Mean Square Error is large compared to the Mean of Response for tryptophan diffusivity ( $72 \%$ of the Mean of Response) and small for glucose diffusivity ( $9 \%$ of the Mean of Response). This means that the fit is not good for tryptophan and is better for glucose. This fit report data backs up the conclusions drawn from the ANOVA, which is that no factor or interaction influenced 
tryptophan diffusivity. Put differently, the prediction model is not a good fit for the tryptophan diffusivity data because the variations are due to error instead of changes in the polymerization conditions. The fit report does show a good fit for glucose, which means that the diffusivity variations between the different bead samples for glucose are not due to random error.

\subsection{Discussion of Diffusivity Results}

The cross-linker concentration has been shown to increase uptake of nitrophenols in work by Peretz and Cinteza [22]. They postulated from their results that the calcium ions present in the electronegative pockets of the gel matrix could have some "channeling effect" on the nitrophenols. The diffusivity was not measured in their research, however the alginate was able to uptake more nitrophenols by adsorption at higher calcium chloride concentrations. In the tryptophan and glucose data, cross-linker concentration itself had no statistical impact on diffusivity for either solute. However, the interaction between cross-linker concentration and alginate concentration was shown to be the most significant factors affecting glucose diffusivity through alginate. The effect on glucose diffusivity for this interaction is negative. This means that as concentration of crosslinker solution increases, the decrease in diffusivity is exacerbated by the changes in alginate concentration. Aslani and Kennedy [7] found that cross-linker concentration did not contribute to variation in permeability measured in their experiment. The levels used in their experiment cover the low cross-linker concentration level, but do not reach as high as $10 \%$ calcium chloride, the highest level used in this work. Their observations showing no impact on permeability for cross-linker concentration could be due to 
different levels tested or is dependent on solute. It is likely that the effect of changes in the polymerization conditions on permeability is solute dependent. The tryptophan and glucose results showed cross-linker concentration did not statistically impact diffusivity on its own, which is consistent with Aslani and Kennedy's findings.

Cross-link time has been shown to be an important factor in some experimental setups and have little to no effect in others. Aslani and Kennedy [7] showed that permeability was negatively affected by increasing cross-link time, but the impact was only seen for very low cross-link times of 1 to 5 minutes. Longer than 5 minutes, the measured permeability and water content in their published study was not affected by the gelation time. Using DOE, Østberg et al. [12] also observed that cross-link time had a negative effect on drug uptake in their system. Presumably, the longer time allowed for the gel matrix to cross-link with the calcium solution, the denser the matrix becomes. The results calculated in this work are consistent with this observation, showing a negative impact of cross-link time on the diffusivity response for both glucose and tryptophan. Cross-link time is considered a main effect for glucose diffusivity, according to the ANOVA. For tryptophan, cross-link time did not statistically impact diffusivity. The levels tested in this work may be inadequate to influence a change in tryptophan diffusivity, and more repeated runs or different levels were not tested.

Alginate concentration was shown to have no significant effect on diffusivity of glucose in alginate membranes in Dembczynski and Jankowski's work [30]. However, their study on glucose used alginate up to a maximum concentration of $1 \% \mathrm{w} / \mathrm{v}$, so it is possible that an effect on glucose diffusivity was not observed in the levels tested in their 
work. Østberg, et al. [12] used DOE to show that alginate concentration had a significant negative effect on the drug uptake. Their work did not study glucose and tryptophan, but the trend may hold when working with other solutes. Alginate concentration itself was not a significant factor for either glucose or tryptophan in these experiments, but the interaction between alginate concentration and cross-linker concentration was shown to be a main effect for glucose diffusivity. It stands to reason, too, that increased alginate concentration in the synthesis of the gel matrix would cause a more dense membrane. This denser membrane network could hinder mass transfer of the solute. In the case of tryptophan, the levels of alginate concentration studied did not significantly affect diffusivity outside of expected experimental errors, according to the ANOVA.

It is clear in reviewing the observations of previous studies that the effect of one factor is not always observed to be influential in all experimental setups. Since diffusivity is a property of a solute in a medium, it is expected that changes in the makeup of the medium (or alginate membrane, in this case) and the solute will make the impact of these experimental factors highly variable. The significant effects and interactions found from the statistical analysis were not the same between glucose and tryptophan diffusivity, even though the polymer membranes were made using the same conditions. For some of the variables used in the experimental design, the levels tested in this work may not have been wide enough to observe a change in the diffusivity response. In particular, alginate concentration had a minor effect on glucose diffusivity, but its interaction with cross-link concentration caused a measurable impact on diffusivity. The 
levels for the cross-linker concentration were only different by two-fold (5\% and 10\%) and a wider spread could contribute to greater variation in the diffusivity.

With such different results between the two solutes, there could be something about the solute molecules themselves that influence these changes. For membranes, solute size is a property that can affect diffusion. The Stokes radius for glucose and tryptophan was calculated using Equation 7 to rule out variations due to molecular size. The calculated Stokes radius and constants used are shown in Table 5. The Stokes radius is similar between the two solutes within two hundredths of a nanometer, which means that both molecules are of similar size when approximated as a hard sphere. Therefore, the solute size is not the reason for the different behaviors of glucose and tryptophan in this study.

Table 5. Stokes radius for glucose and tryptophan, calculated using Equation 7.

\begin{tabular}{|l|l|l|}
\hline & Glucose & Tryptophan \\
\hline Molecular Weight (g/mol) & 180.15 & 204.23 \\
\hline Stokes Radius, r (nm) & 0.415 & 0.436 \\
\hline Constants & $\begin{array}{l}\mathrm{N}=6.02 \times 10^{23} \\
\rho=1 \mathrm{~g} / \mathrm{cm}^{3}\end{array}$ \\
\hline
\end{tabular}

The membranes made from alginate are chemical membranes, so size is not the only reason that molecules can be excluded. There can be interactions between atoms in the chemical structure of the membrane that repel or attract molecules. It is possible that solute molecules could adsorb to the surface due to chemical interactions and very little diffuses into the matrix of the polymer. If this were the case, then measured solute over time would be the rate of desorption. For glucose, the prediction model used by JMP showed a good fit for the diffusivity data. Therefore, the it is likely that diffusion is the 
main mass transfer phenomenon for glucose in this system. However, tryptophan did not show a good fit for diffusivity with the prediction model. Tanaka et al. [15] studied tryptophan diffusivity into alginate beads initially free of solute. Their results found very little difference between diffusivity of tryptophan in water compared to their measured diffusivity into $2 \% \mathrm{w} / \mathrm{v}$ alginate beads. Different alginate concentrations or cross-linking conditions were not tested on tryptophan in their study. Tanaka et al. concluded that tryptophan could freely diffuse into the alginate bead. However, their study assumed that diffusion was the main mass transfer phenomena at work. It is possible that the experimental levels are unsuitable to influence change in diffusivity or that there are mass transfer mechanisms other than diffusion happening in the system, such as desorption. The poor fit of the tryptophan diffusivity data against the prediction model could be an indication that the assumptions made about the mass transfer in this system are too simplified. A fit of tryptophan data similar to the data taken in this work to many different adsorption isotherm models would be an appropriate way to discover if adsorption yields a better fit than diffusion. Desorption is not the focus of this study and so such analysis was not done.

\subsection{Initial Concentration Results}

It was observed that different bead samples had different calculated initial concentrations. This system is modeled by Equation 3, which assumes a sphere of uniform initial concentration, $\mathrm{C}_{0}$, is in a bulk solution initially free of solute. The solute diffuses out of the sphere and into the bulk solution, and the concentration of the solute in the bulk solution, $\mathrm{C}_{\mathrm{t}}$, is measured over time, $\mathrm{t}$. The initial concentration for each 
diffusion trial was calculated using Equation 5. It was observed that there are variations between the initial concentrations across different bead samples. It was postulated that there is a concentration limit inside the alginate beads, which could be dependent on the solute and the membrane composition. This concentration limit could be evaluated by comparing the concentration of solute inside the alginate beads after overnight incubation in a high concentration solution. The alginate beads were all incubated in the same high concentration of solute overnight in order to saturate the beads with solute before diffusion trials. Calculation of the initial concentration from Equation 5 was already necessary to generate the diffusivity results. The statistical analysis used to compare diffusivity across different bead samples was repeated for the initial concentrations.

Table 6 shows the calculated initial concentrations for all bead samples. Beads were incubated in a high concentration solution overnight prior to diffusion trials. The concentration of the overnight solution is listed in Table 6 . The solubility of the solutes in water is also presented for reference. For glucose, Table 6 shows that the initial concentration for all bead samples is close to or exceeding the overnight solution concentration. It is not expected that the concentration in the alginate beads could be higher than the saturating solution. There is also noise in the data from factors that can and cannot be controlled that will cause variations in the calculation. More repeated runs would help to smooth the noise in the data. For tryptophan, the initial concentrations are all well below the overnight solution concentration. The dependence on initial concentration on the membrane composition was analyzed by repeating the comparative analysis done on diffusivity results. 
Table 6. Initial concentration for both tryptophan and glucose for all bead samples A through $\mathrm{H}$, compared to the overnight solution.

\begin{tabular}{|c|c|c|c|c|c|}
\hline \multicolumn{4}{|c|}{ Polymerization Conditions } & \multirow[b]{2}{*}{$\begin{array}{l}\text { Glucose } \\
\text { Runs } \mathrm{C}_{0} \\
(\mathrm{mg} / \mathrm{mL})\end{array}$} & \multirow[b]{2}{*}{$\begin{array}{l}\text { Tryptophan } \\
\mathrm{C}_{0} \\
(\mathrm{mg} / \mathrm{mL})\end{array}$} \\
\hline $\begin{array}{l}\text { Bead } \\
\text { Sample } \\
\text { Name }\end{array}$ & $\begin{array}{l}\text { Alginate } \\
\text { Conc. } \\
(w / v \%)\end{array}$ & $\begin{array}{l}\mathrm{CaCl}_{2} \\
\text { Conc. } \\
(\mathrm{w} / \mathrm{v} \%)\end{array}$ & $\begin{array}{l}\text { Crosslink } \\
\text { Time } \\
(\min )\end{array}$ & & \\
\hline A & 1.5 & 5 & 15 & 46.93 & 0.08 \\
\hline $\mathrm{B}$ & 2.5 & 5 & 15 & 46.62 & 0.16 \\
\hline $\mathrm{C}$ & 1.5 & 10 & 15 & 38.59 & 0.16 \\
\hline $\mathrm{D}$ & 2.5 & 10 & 15 & 40.45 & 0.16 \\
\hline $\mathrm{E}$ & 1.5 & 5 & 60 & 35.86 & 0.16 \\
\hline $\mathrm{F}$ & 2.5 & 5 & 60 & 35.71 & 0.16 \\
\hline $\mathrm{G}$ & 1.5 & 10 & 60 & 40.61 & 0.18 \\
\hline $\mathrm{H}$ & 2.5 & 10 & 60 & 40.08 & 0.15 \\
\hline \multicolumn{4}{|c|}{$\begin{array}{l}\text { Concentration of } \\
\text { overnight solution }\end{array}$} & 40 & 0.33 \\
\hline & & \multicolumn{2}{|c|}{$\begin{array}{r}\text { Solubility in Water } \\
{[31,32]}\end{array}$} & 133 & 13.4 \\
\hline
\end{tabular}

The comparative analysis on initial concentration is to determine if the ability of the solutes to diffuse into the membrane during their overnight incubation is influenced by the polymerization conditions of the membranes. Figure 27 shows the measured effect of each factor and interaction on initial concentration for glucose and tryptophan, calculated using Equation 9. Very few factors and interactions are of similar magnitude between glucose and tryptophan. 


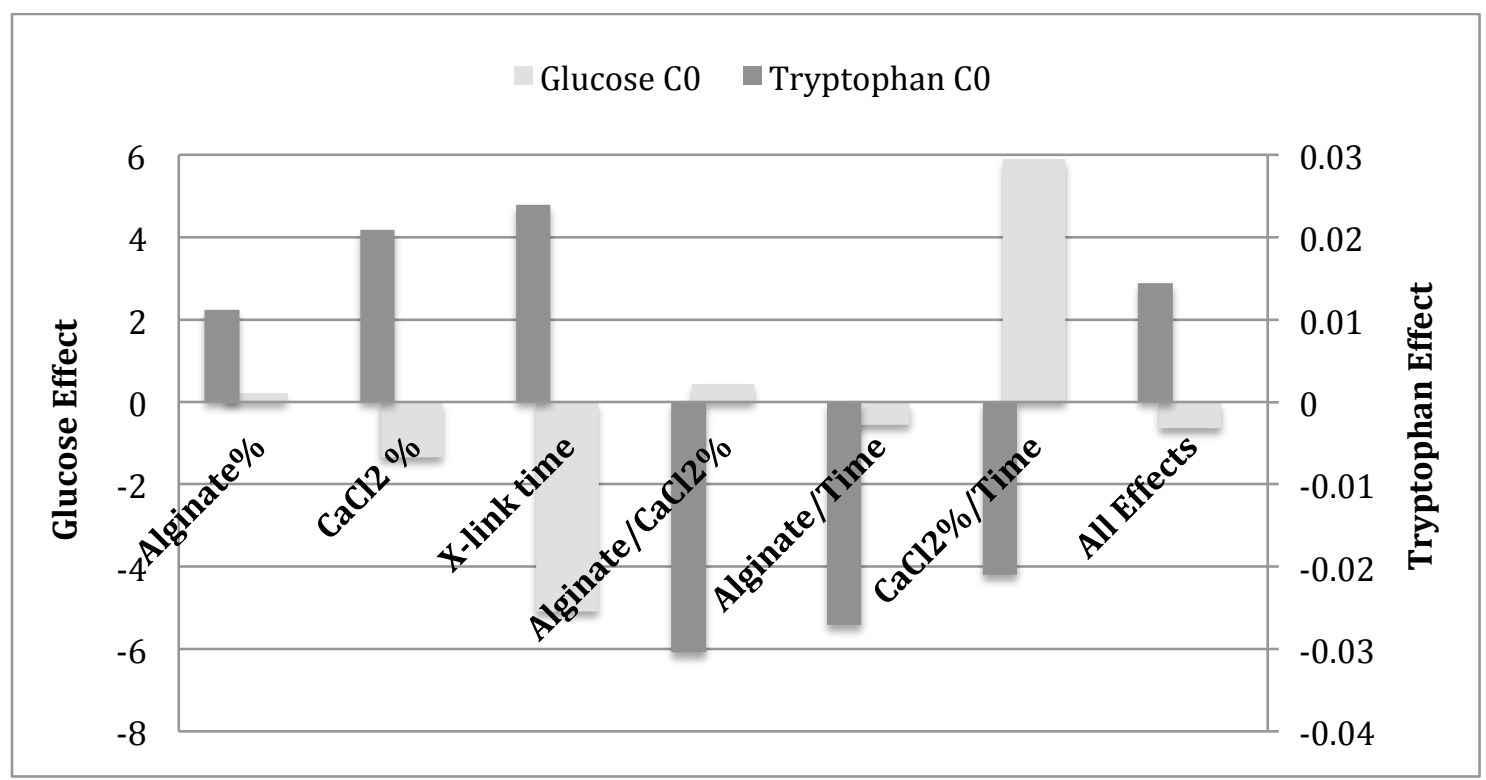

Figure 27. Factors and interactions of glucose and tryptophan on initial concentration, $\mathrm{C}_{0}$.

Prediction profiles were generated in JMP to visualize the effects on initial concentration with the slope of a line. Figures 28 and 29 show the prediction profiles for tryptophan and glucose, respectively. Tryptophan shows a positive effect on initial concentration for all factors. The combined results for initial concentration from both glucose diffusion runs shows a net zero effect for alginate, a slight negative effect for cross-link concentration, and a much steeper negative effect for cross-link time.

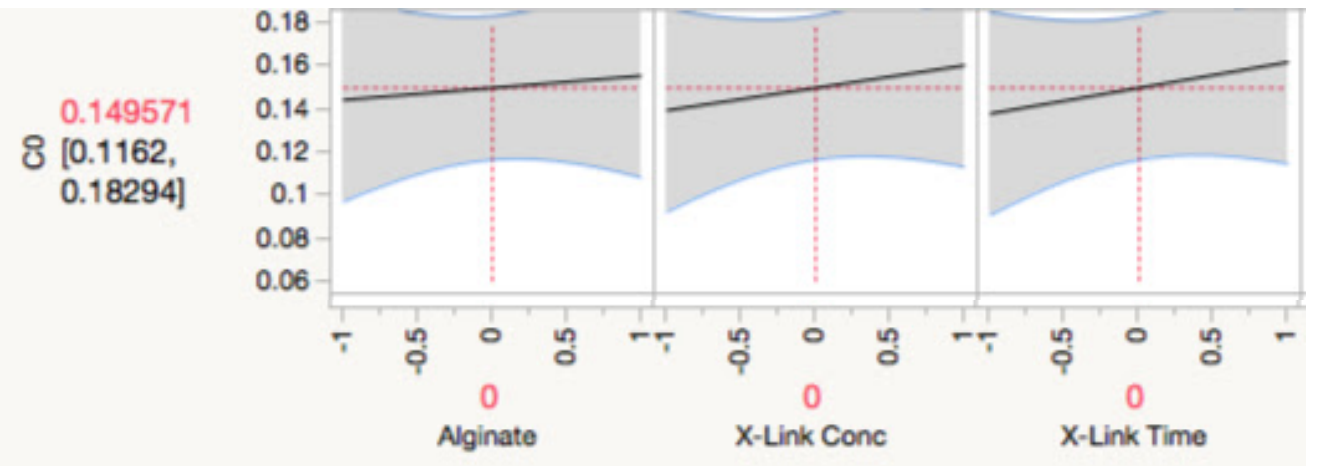

Figure 28. Prediction profile for tryptophan initial concentration created using JMP. 

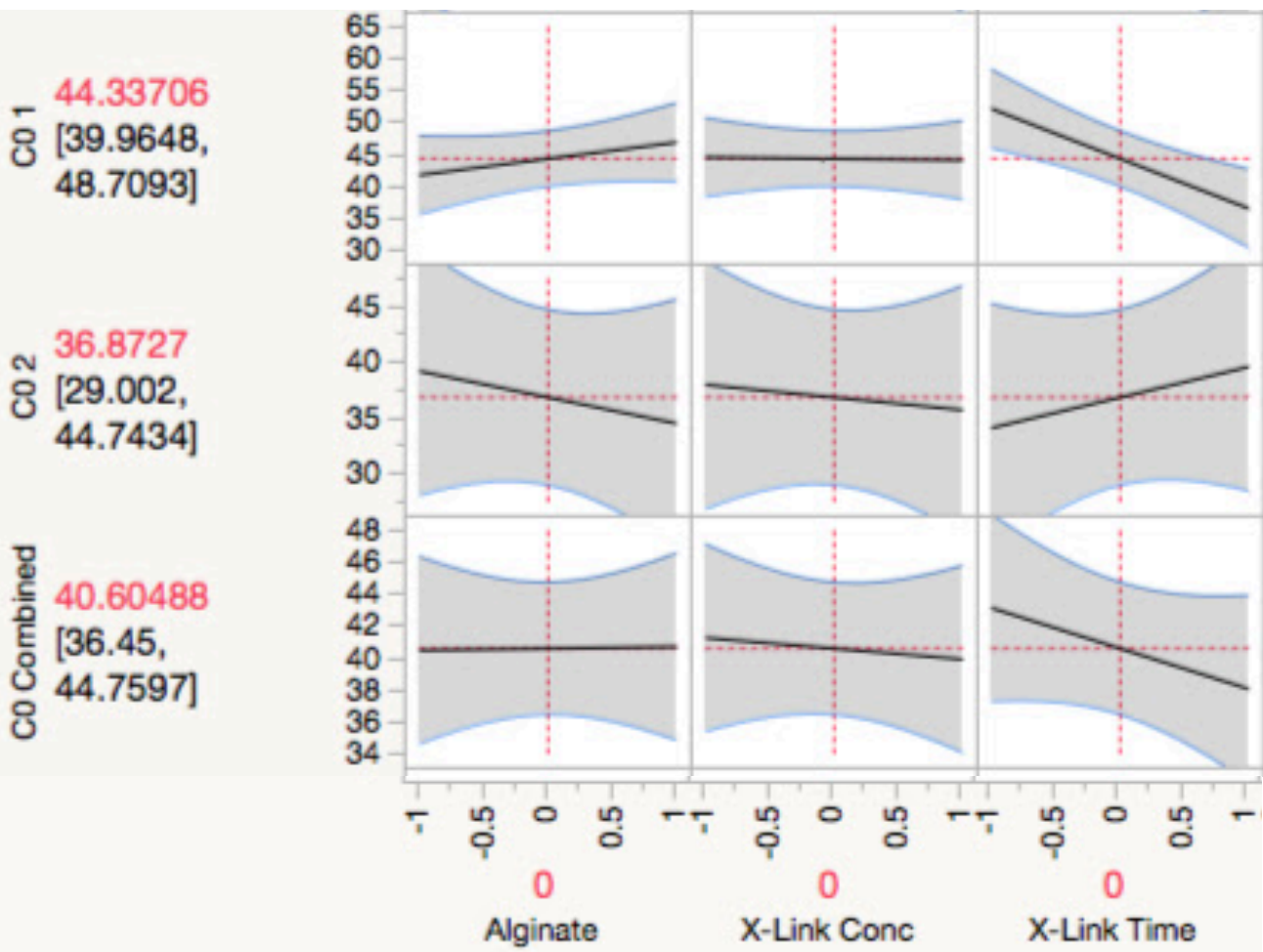

Figure 29. Prediction profile for glucose initial concentration created using JMP.

To determine factors and interactions that vary outside of normal error, half-normal probability plots for tryptophan and glucose initial concentration were created and are shown in Figures 30 and 31, respectively. These plots show factors and interactions that vary outside of normal error, as in the results for diffusivity. Tryptophan initial concentration shows all factors and interactions fit on a line in Figure 30. In Figure 31, all factors and interactions for glucose initial concentration fit on a line save for crosslink time and the interaction between cross-link concentration and time. The statistical impact of all these data can be analyzed using ANOVA. 


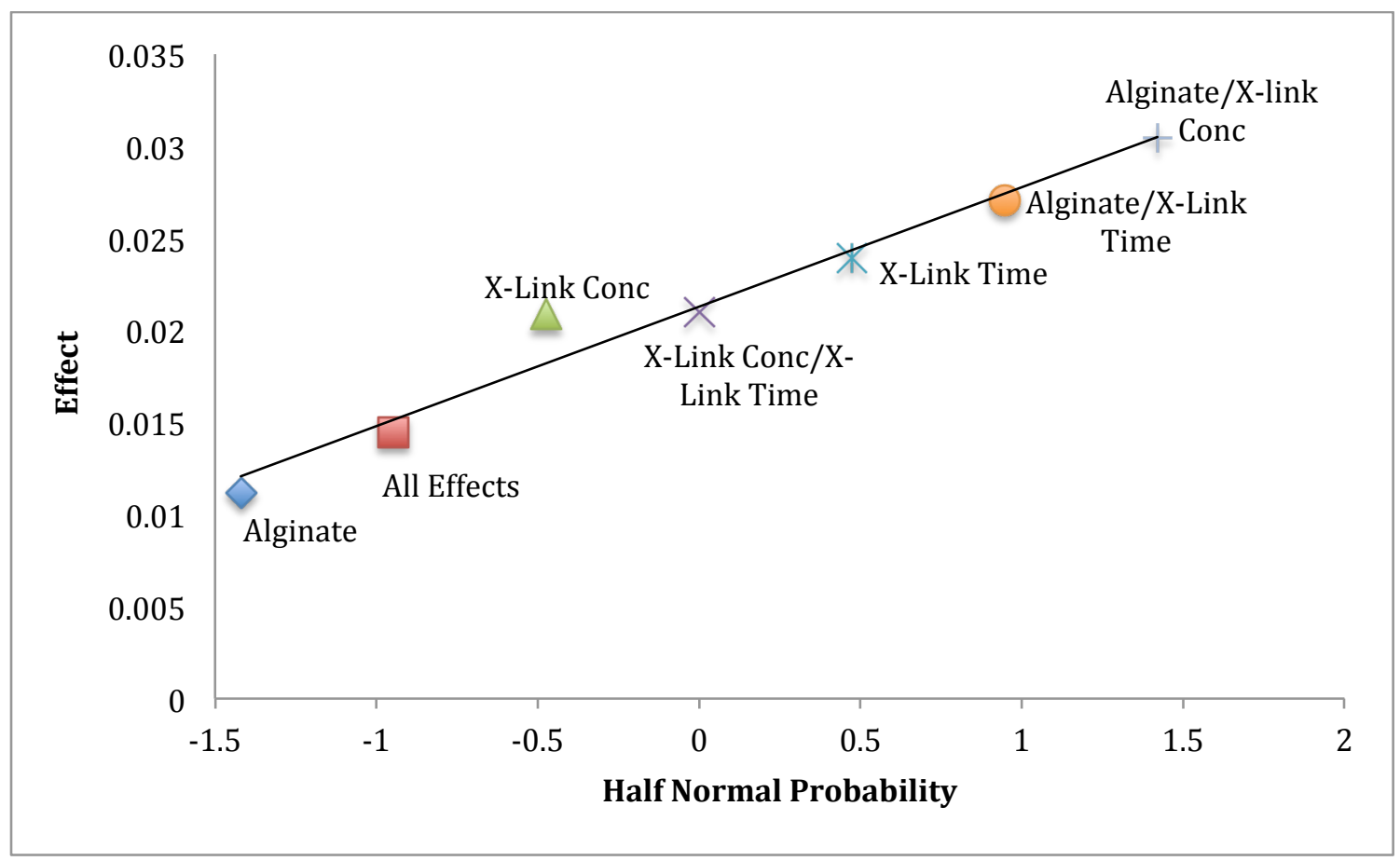

Figure 30. Half-normal probability plot of tryptophan initial concentration, $\mathrm{C}_{0}$, effects and interactions.

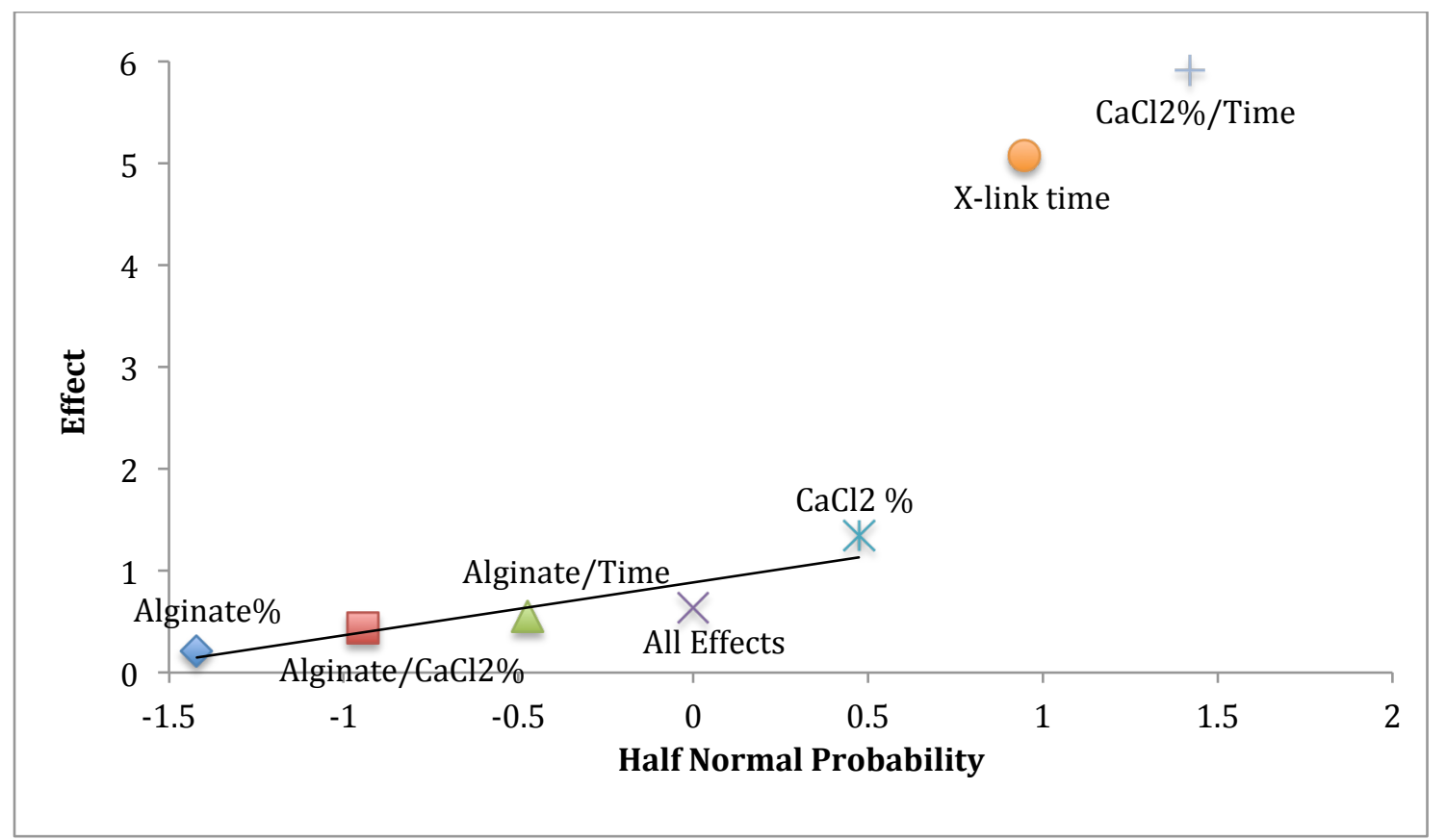

Figure 31. Half-normal probability plot of glucose initial concentration, $\mathrm{C}_{0}$, effects and interactions. 
ANOVA was performed using JMP and tables are presented in Appendix A, Tables 14-17. The statistical analysis showed that all factors and interactions for the initial concentration of tryptophan had a $\mathrm{P}$ value much greater than 0.05 . Therefore, no factor or interaction influences the initial concentration of tryptophan in the alginate beads outside of normal error. For glucose initial concentration, all factors and interactions had a $\mathrm{P}$ value greater than 0.05 , but the $\mathrm{P}$ values for cross-link time and the interaction between cross-link concentration and time are the lowest. These two sources showed a P value less than $8 \%$, which means the variations have a low probability of being due to normal error. This explains why these two effects are off the line on the half-normal probability plot in Figure 31, compared to all other effects. For subsequent discussion of initial concentration results, the cross-link time and the interaction between cross-link concentration and time will be considered main effects impacting the glucose initial concentration.

The fit report statistics of the prediction model obtained from JMP are shown for tryptophan and glucose initial concentration, respectively, in Appendix A, Tables 18-19. The R-squared, adjusted R-squared, root mean square error, mean of response, and the number of observations used are reported. Using initial concentration as the response, the R-squared is much higher for tryptophan at 0.94 , compared to the poor fit obtained for diffusivity. The Root Mean Square Error is also a smaller percentage of the Mean of Response at $13 \%$, so the variation between initial concentrations of tryptophan is due to normal error. The fit on glucose initial concentration is also good with a high R-squared of 0.93 and a low Root Mean Square Error to Mean of Response ratio at 15\%. The error 
to Mean of Response ratio is not so low that the normal error is insignificant, but the prediction model is a better fit for both solutes compared to the fit on diffusivity results.

\subsection{Discussion of Initial Concentration Results}

The initial concentration calculated in this experimental setup is a measure of how well the solute molecules can equilibrate with the polymer membrane. Alginate beads were allowed to incubate in the high concentration solution overnight, which should be sufficient for equilibrium to be reached. The goal of comparing these calculated initial concentration values was to determine if differences between the bead samples were due to the polymerization conditions. A denser gel matrix could impede the allowed concentration in the alginate beads, due to occlusion of the solute molecules in a more closely packed matrix. This response of initial concentration is difficult to compare to literature because solutes diffusing out of alginate beads and into the bulk is not often studied. Much of the work on alginate beads focuses on diffusion of solutes from the bulk into the alginate beads, which are initially free of solute. This is especially useful for waste sequestration applications.

From the half normal plot in Figures 30, no factor or interaction showed deviation off the straight line for tryptophan. In Figure 31, cross-link time and the interaction between cross-link concentration and time showed significant deviation from the straight line for glucose. ANOVA results for tryptophan and glucose backed up these observations. Tryptophan initial concentration was unaffected by any changes in polymerization condition tested in this work. The fit reports showed that the prediction model was a good fit for both glucose and tryptophan. This means that the model used for ANOVA to 
determine if variations in a factor or interaction is significant is a good fit to the data. In other words, the ANOVA results have high confidence to determine if variations are due to normal error or due to a significant factor.

The results of initial concentration presented in this study would only be able to explain if there is a hindrance to a solute's ability to equilibrate with the bulk solution due to the membrane composition. For tryptophan, the data again shows that none of the factors at these levels had any impact outside of normal error on the initial concentration. It is interesting to note that while glucose initial concentration was similar to the overnight solution concentration in Table 6, tryptophan initial concentration stayed below the overnight solution concentration for all bead samples by about half. In the paper by Tanaka et al. [15], diffusion of glucose and tryptophan was measured into alginate beads and were shown to be similar to the diffusivity of these solutes in water. If glucose can diffuse freely into or out of the alginate spheres as reported in their results, then it is expected that the initial concentration of glucose at all bead samples are close to the concentration of the overnight solution, because it is able to equilibrate. For tryptophan, the initial concentration results do not agree with this observation. The initial concentration of tryptophan was much lower than the overnight solution. If it could freely diffuse in and out of the gel matrix, an initial concentration closer to equilibrium would be expected. These results could mean that there is a hindrance in tryptophan's ability to equilibrate across the alginate membrane in the overnight solution. It is possible that diffusion is not the only mass transfer mechanism at work for tryptophan. Tanaka et al. did not observe this phenomenon because their study only measured 
diffusion from the bulk into the alginate beads; diffusion of concentrated tryptophan out of the alginate beads and into the bulk fluid was not performed in their study.

For glucose, the cross-link time was determined to be a main factor for the initial concentration response. Tanaka et al. [15] showed that glucose could diffuse freely into and out of alginate beads. However, their experimental setup did not vary cross-link time, and instead kept the cross-link time constant at 2 hours for all alginate beads generated for the study. In general, the diffusion can be hindered by higher cross-link time depending on the experimental setup, according to Tønnesen and Karlsen [21]. Indeed, a greater cross-link time had a negative impact on the initial concentration for glucose as reported in the prediction profile in Figure 29 and the bar chart of calculated effects in Figure 27. This means that beads that were cross-linked for 60 minutes were able to equilibrate to an average lower concentration in the overnight solution compared with the beads cross-linked for 15 minutes.

The interaction between cross-link concentration and time was shown to be a main effect on the initial concentration of glucose. The interaction positively influenced the initial concentration of glucose. This means that cross-linker concentration and crosslink time had a cooperative effect, resulting in higher average initial concentration when both factors were at the high level. Cross-linker concentration has been shown to allow decreased uptake of materials by Østberg et al. [12], Holte et al. [13], and Peretz and Cinteza [22]. There is less reference material available using a DOE approach to study the interactions between factors for mass transfer in alginate. However, the study by Østberg et al. [12] uses the some of the same experimental conditions as this work, 
although with different solutes. Their results did show a positive impact on mass transfer for the interaction between cross-linker concentration and time. This interaction was not deemed statistically significant in their study, but the two factors did show a cooperative effect, which is consistent with the reported results in this study for glucose.

\subsection{Recommendations for Design of Experiments}

It is important to consider that the data collected in this work is not consistent with theory. The measured diffusivity was higher than the values reported in water for some of the diffusion trials, which is contradictory to what is expected. The variations between the bead samples were analyzed to look at relative differences and provide an outline for future work. The results from the full-factorial analysis are not directly consistent with the literature, but similar trends are observed. Based on the results presented, it is not recommended to run similar levels to calculate diffusivity for tryptophan. None of the polymerization conditions influenced the diffusivity, and there are indications that assuming diffusion is the only mass transfer mechanism at work in the system is not sound. This hypothesis could be tested by collecting similar concentration versus time data for tryptophan and doing a fit to different adsorption isotherm models and compare with diffusion.

For glucose, there is evidence in the results presented and the literature that crosslinker concentration is an important factor. Higher cation concentration has been shown to decrease the permeability [7]. This factor alone did not influence the diffusivity, but the interaction between alginate concentration and cation concentration did negatively influence glucose diffusivity. A wider spread with more levels for cross-linker 
concentration would broaden the understanding of this factor's influence on glucose diffusivity.

No significant influence of alginate concentration alone was observed in these experiments, and could be a topic of further study. For alginate concentration, the highest concentration of alginate tested in many papers is $4 \% \mathrm{w} / \mathrm{v}$. Tanaka et al. [15] showed that the higher alginate concentration decreased the diffusivity. However, their study did not report diffusivity calculated for all of the $2 \%$ and $4 \%$ alginate beads for every solute. In reality, the alginate dissolves in water with much difficulty and alginate solution at much higher than $2.5 \% \mathrm{w} / \mathrm{v}$ can become clogged in the syringe, depending on the materials used. In order to reduce time in the lab dissolving alginate solid and creating the beads, it is not recommended to exceed $2.5 \% \mathrm{w} / \mathrm{v}$ alginate.

Lastly, the cross-link time did affect the glucose diffusivity and initial concentration. This is consistent with literature observations by Aslani and Kennedy [7]; however, their work did not see a change in permeability with cross-linking times longer than 5 minutes. From these observations, the levels for this work were expanded to higher than previously tested. In order to test the observations from by Aslani and Kennedy, a crosslink time of 60 minutes was used for the high level. Cross-linking time is a parameter of interest to study because the literature is not in agreement whether or not it is an important factor [21]. Shorter cross-linking time is operationally more difficult to control and keep consistent, but would likely lead to the most dramatic changes compared to an hour or more of cross-linking. 
In general, mass transfer in alginate gel is a prevalent experiment with a long history in scientific publications. There are trends in mass transfer properties with respect to the membrane composition, but the levels need to be tuned to the solute. To build on this work, it is critical to run experiments that are consistent with the math. In particular, adequate mixing is the only way to ensure that measurement of concentration in the bulk is a suitable approximation for the concentration at the surface of the spheres.

Additionally, the sampling conditions need to be carefully planned to ensure a constant volume assumption is still valid. Therefore, it is not recommended to remove more than $10 \%$ of the total volume. 


\section{CHAPTER SIX}

\section{CONCLUSIONS}

\subsection{Addressing Objectives}

The first objective for this work was to establish an experimental protocol to make alginate beads with uniform size, including finding levels that work well for the experiment. The method used in this work was developed from many research papers working with alginate beads, and continues to be a reliable and inexpensive method to achieve adequate uniformity. The consistent alginate bead diameter showed this objective was met. It is possible that the uniformity of the alginate beads could be improved by ensuring that the distance between the syringe and cross-linking solution remains constant. According to MØrch et al. [6], this factor could be important to achieving consistent spheres. This was not specifically controlled as constant in this work, and may be responsible for some of the variation in the diameter of alginate beads.

The second objective of this study was to measure the change in concentration of solutes over time diffusing out of the alginate beads. The sampling conditions were carefully planned to ensure assumptions in the modeling equations were sound. The total volume of samples removed from the beaker over the course of each run was designed to stay at $10 \%$ or less of the total bulk volume. The ratio of beads to bulk fluid was scaled to make this sampling and constant volume assumption possible. The pilot time test runs were used to plan the sampling times so that the minimal amount of fluid was removed. Since all variations were made in the alginate polymerization conditions, all of the mass transfer data collection was run under identical conditions to minimize variation. The 
data collected follows the expected theoretical trend, and showed a good nonlinear fit, so this objective was met.

The third objective of this study was to calculate diffusivity from the collected concentration versus time data. This was accomplished by studying the literature of similar experiments. In particular, Crank's book [18] on the mathematics of diffusion is an invaluable resource because it develops Fick's law equations to many system geometries. An Excel sheet with macro was made to perform the Goal Seek function on multiple cells simultaneously to solve for diffusivity across all data points. Mathematical software such as MATLAB or Mathematica could also be used for this purpose. The diffusivities for all runs were obtained and a consensus diffusivity for each bead sample and each solute was calculated.

The fourth and final objective in this work was to calculate the main effects in this experimental matrix. The factors and levels chosen in this study were among commonly studied conditions found in the literature. In addition, preliminary tests of this experiment have been performed in the chemical engineering senior undergraduate lab course at San José State University, although the mathematical analysis was not as in depth. This objective was met by using standard statistical analysis methods in DOE. It was expected that alginate concentration would be more impactful in the diffusivity given the survey of literature. It is surprising that this factor was not shown to be a main effect, given that the viscosity change between the $1.5 \%$ and $2.5 \% \mathrm{w} / \mathrm{v}$ alginate solutions was noticeable when stirring the solutions. At different levels or solutes, it is possible that factors that did not significantly impact the response in this experimental matrix could be 
a main effect. It is also surprising that tryptophan showed no main effects for the experimental responses, given prior observations published by Tanaka et al. [15]. From the results, there are indications that the assumptions for this experiment may not be applicable to tryptophan, and could be a subject of future work.

The results here lend insight into how to run the experiment in the future, which will aid the students in the senior lab course. Appendix B contains a recommended experimental protocol for the senior lab course based on literature surveyed and data taken in this study. This experiment is appealing for a senior lab course in chemical engineering because of the breadth of topics covered including polymerization, membranes, mass transfer, DOE, working with partial differential equations, and laboratory methods using the spectrophotometer. Additionally, the experiment can be repeated using inexpensive consumables and analytical equipment already available in the current unit operations chemical engineering laboratory at San José State University.

In conclusion, changing polymerization conditions of the alginate beads was found to affect the diffusivity of the solute, but the changes are solute dependent. The difference in glucose and tryptophan responses is not likely due to the size of the solute. It is proposed that the change in concentration over time for tryptophan is not due to diffusion alone, but possibly has some contribution by desorption of the solute from the surface of the polymer. This hypothesis is only reasonable if the desorption can also be modeled as an exponential function, and would need to be tested as further work. At minimum, for the levels tested in this work, tryptophan diffusivity was not influenced, and more drastic changes could be seen with a different set of levels for the three factors tested. A 
response of initial concentration was also analyzed, and showed that cross-link time and the interaction of cross-link concentration and time of the alginate membrane had an impact on the initial concentration of glucose. For tryptophan, no factors or interactions influenced the initial concentration significantly.

\subsection{Possible Future Work}

This work has potential for further development. In particular, varying the tested polymerization conditions with a wider spread of levels in order to observe a change for tryptophan. It is unknown if the polymerization conditions will influence any change in diffusivity for tryptophan. If there are more mass transfer phenomena at play with tryptophan, the assumptions made in this experiment would not be applicable and a more complex system and equation would need to be developed. The concentration over time data taken in a setup similar to this work could be fit to many different desorption isotherm models to test the desorption hypothesis. It is likely that more than the effects shown in this study could influence glucose. The dynamic range of alginate concentration that influences glucose diffusivity, for example, could be a topic of study to see more dramatic changes in the diffusivity.

Other factors that may affect diffusion or polymerization are $\mathrm{pH}$ and temperature. Castro et al. [5] observed the effect of $\mathrm{pH}$ and temperature on diffusion. These variables are appealing as potential factors for a future experiment because they would be operationally easy to keep constant. Buffers at varied $\mathrm{pHs}$ could be used to determine the effect of $\mathrm{pH}$ on the diffusivity, which could be impactful when working with protein 
solutes as their structure is dependent on $\mathrm{pH}$. Temperature could also be controlled using a water bath on the heated stir plate that is already used to keep the system well mixed.

The ratio of volume of beads to bulk solution was held constant at 1 to 10 in this study. This ratio is related to a variable in Equation 3, called $\alpha$, so would directly impact the calculation of diffusivity of the solute. It would be interesting and operationally simple to vary this ratio and calculate its impact on diffusivity. Changing this ratio of volumes would indeed impact the diffusivity, but too small of a ratio may not show a measurable increase of solute concentration over time. Similarly, too large of a ratio leaves less total volume in the bulk, which can cause problems with removal of too much fluid over time.

In this study, glucose and tryptophan were used because there are reference materials for the diffusion of these solutes [15]. Other solutes that have been used in the literature for similar studies are nitrophenol, ethanol, immunoglobulin, copper ions, creatinine, and some dyes. To modify this experimental setup to use a different solute, all that is needed is to find a reliable analytical method to measure the concentration of the solute. In choosing a new solute for study, rules of thumb put forth in a review by Stewart [20] can be taken into consideration to ensure solute molecules are likely to diffuse well through the alginate matrix. As in this study, the polymerization conditions of the hydrogel can affect the diffusivity but will be dependent on the solute properties and size. 


\section{REFERENCES}

1. T. Vermonden, R. Censi and W.E. Hennink, "Hydrogels for protein delivery," Chem. Rev, 112, pp. 2853-2888 (2012).

2. G.T. Grant, E.R. Morris, D.A. Rees, P.J.C. Smith, and D. Thom, "Biological interactions between polysaccharides and divalent cations: the egg-box model," FEBS Letters, 32, pp. 195-198 (1973).

3. S.K. Papageorgiou, E.P. Kouvelos and F.K. Katsaros, "Calcium alginate beads from Laminaria digitata for the removal of $\mathrm{Cu}+2$ and $\mathrm{Cd}+2$ from dilute aqueous metal solutions," Desalination, 224, pp. 293-306 (2008).

4. N. Wang, G. Adams, L. Buttery, F.H. Falcone and S. Stolnik, "Alginate encapsulation technology supports embryonic stem cells differentiation into insulinproducing cells," J. of Biotechnol., 144, pp. 304-312 (2009).

5. G.R. Castro, B. Panilaitis, E. Bora and D.L. Kaplan, "Controlled release biopolymers for enhancing the immune response," Molecular Pharmaceutics, 4, pp. 33-46 (2007).

6. Y.A. MØrch, I. Donati, B.L. Strand and G. Skjåk-Bræk, "Effect of $\mathrm{Ca}^{2+}, \mathrm{Ba}^{2+}$, and $\mathrm{Sr}^{2+}$ on alginate microbeads, ” Biomacromolecules, 7, pp. 1471-1480 (2006).

7. P. Aslani and R.A. Kennedy, "Studies on diffusion in alginate gels. I. Effect of crosslinking with calcium or zinc ions on diffusion of acetaminophen, ” J. of Controlled Release, 42, pp. 75-82 (1996).

8. E. Schuster, J. Eckardt, A. Hermansson, A. Larsson, N. Lorén, A. Altskär and A. Ström, "Microstructural, mechanical and mass transport properties of isotropic and capillary alginate gels," Soft Matter, 10, pp. 357-366 (2014).

9. NIST/SEMATECH e-Handbook of Statistical Methods, section 5.5.9.8 [Online]. Available at http://www.itl.nist.gov/div898/handbook/ (accessed 17 February 2017). Web Handbook.

10. G.E.P. Box, Statistics for Experimenters: Design, Innovation, and Discovery, $2^{\text {nd }}$ ed. (John Wiley \& Sons, Inc. Hoboken, New Jersey, 2005), pp. 174-200. 
11. J.P. Verma, Repeated Measures Design for Empirical Researchers, $1^{\text {st }}$ ed. (John Wiley \& Sons, Inc. Hoboken, New Jersey, 2015), pp. 4-20.

12. T. Østberg, L. Vesterhus and C. Graffner, "Calcium alginate matrices for oral multiple unit administration: II. Effect of process and formulation factors on matrix properties, ” Int. J. Pharm., 97, pp. 183-193 (1993).

13. Ø. Holte, H.H. TØnnesen and J. Karlsen, "Measurement of diffusion through calcium alginate gel matrices,” Pharmazie, 61, pp. 30-34 (2006).

14. E. Chan, T. Lim, W. Voo, R. Pogaku, B.T. Tey and Z. Zhang, "Effect of formulation of alginate beads on their mechanical behavior and stiffness," Particuology, 9, pp. 228-234 (2011).

15. H. Tanaka, M. Matsumura and I.A. Veliky, "Diffusion characteristics of substrates in Ca-alginate gel beads,” Biotechnol. Bioeng., 26, pp. 53-58 (1984).

16. J. Ha, C.R. Engler and S.J. Lee, "Determination of diffusion coefficients and diffusion characteristics for chlorferon and diethylthiophosphate in Ca-alginate gel beads," Biotechnology and Bioengineering, 100, pp. 698-706 (2008).

17. S. Gabardo, R. Rech and M.A. Zachia Ayub, "Determination of lactose and ethanol diffusion coefficients in calcium alginate gel spheres: predicting values to be used in immobilized bioreactors, ”J. Chem. Eng. Data, 56, pp. 2305-2309 (2011).

18. J. Crank, The Mathematics of Diffusion, $2^{\text {nd }}$ ed. (Oxford University Press. London, England, 1975), pp. 89-96.

19. H. Kakita and H. Kamishima, "Some properties of alginate gels derived from algal sodium alginate,” J. Appl. Phycol., 20, pp. 93-99 (2008).

20. P.S. Stewart, "A review of experimental measurements of effective diffusive permeabilities and effective diffusion coefficients in biofilms," Biotechnology and Bioengineering, 59, pp. 261-272 (1998).

21. H.H. Tønnesen and J. Karlsen, "Alginate in drug delivery systems," Drug Dev. Ind. Pharm., 28, pp. 621-630 (2002). 
22. S. Peretz and O. Cinteza, "Removal of some nitrophenol contaminants using alginate gel beads," Colloids and Surfaces, 319, pp. 165-172 (2008).

23. I. Donati, Y.A. MØrch, B.L. Strand, G. Skjåk-Bræk and S. Paoletti, "Effect of elongation and alternating sequences on swelling behavior and large deformation properties of natural alginate gels, ”J. Phys. Chem., 113, pp. 12916-12922 (2009).

24. J.R. Mitchell and J.M.V. Blanshard, "Rheological properties of alginate gels," J. of Texture Stud., 7, pp. 219-234 (1976).

25. S.K. Bajpai and S. Sharma, "Investigation of swelling/degradation behavior of alginate beads crosslinked with $\mathrm{Ca} 2+$ and Ba2+ions, " React. Funct. Polym., 59, pp. 129-140 (2004).

26. A.L. Hines and R.N. Maddox, Mass Transfer: Fundamentals and Applications, $1^{\text {st }}$ ed. (Prentice Hall. Englewood Cliffs, New Jersey, 1985), pp. 60-86.

27. I. Bass, Six Sigma Statistics with Excel and Minitab, $1^{\text {st }}$ ed. (McGraw-Hill Companies, Inc. New York, New York, 2007), pp. 276-288.

28. F.L. Dullien, Porous Media: Fluid Transport and Pore Structure, $2^{\text {nd }}$ ed. (Academic Press, 1992).

29. J.R. Taylor, An Introduction to Error Analysis, $2^{\text {nd }}$ ed. (University Science Books. Sausalito, California, 1982), pp. 166-169.

30. R. Dembczynski and T. Jankowski, "Characterisation of small molecules diffusion in hydrogel-membrane liquid-core capsules,” Biochem. Eng. J., 6, pp. 41-44 (2000).

31. Sigma Product Information. $D-(+)-G l u c o s e$ [Online]. Available at https://www.sigmaaldrich.com/content/dam/sigmaaldrich/docs/Sigma/Product_Information_Sheet/2/g5400pis.pdf (accessed 30 August 2017). Posted pdf file.

32. PubChem Open Chemistry Database. L-tryptophan [Online]. Available at https://pubchem.ncbi.nlm.nih.gov/compound/L-tryptophan (accessed 30 August 2017). WWW article. 
33. R. Russo, M. Malinconico and G. Santagata, "Effect of cross-linking with calcium ions on the physical properties of alginate films," Biomacromolecules, 8, pp. 31933197 (2007). 


\section{APPENDIX A}

\section{SUPPLEMENTARY TABLES}

Table 7. Bead diameter measurements and calculated average, standard deviation, and standard errors.

\begin{tabular}{|l|r|r|r|r|r|r|r|r|}
\hline Run & $\begin{array}{c}\mathrm{A} \\
(\mathrm{mm})\end{array}$ & $\begin{array}{c}\mathrm{B} \\
(\mathrm{mm})\end{array}$ & $\begin{array}{c}\mathrm{C} \\
(\mathrm{mm})\end{array}$ & $\begin{array}{c}\mathrm{D} \\
(\mathrm{mm})\end{array}$ & $\begin{array}{c}\mathrm{E} \\
(\mathrm{mm})\end{array}$ & $\begin{array}{c}\mathrm{F} \\
(\mathrm{mm})\end{array}$ & $\begin{array}{c}\mathrm{G} \\
(\mathrm{mm})\end{array}$ & $\begin{array}{c}\mathrm{H} \\
(\mathrm{mm})\end{array}$ \\
\hline Tryptophan & 3.71 & 3.49 & 3.48 & 3.74 & 3.44 & 4.07 & 3.64 & 3.47 \\
\hline Tryptophan & 3.78 & 3.62 & 3.44 & 3.74 & 3.66 & 3.98 & 3.33 & 3.51 \\
\hline Tryptophan & 3.99 & 4.01 & 3.47 & 3.86 & 3.61 & 3.88 & 3.58 & 3.26 \\
\hline Tryptophan & 3.76 & 3.71 & 3.57 & 3.58 & 3.62 & 4.03 & 3.49 & 3.28 \\
\hline Tryptophan & 3.72 & 3.41 & 3.5 & 3.66 & 3.53 & 4.06 & 3.57 & 3.33 \\
\hline Glucose Run 1 & 4.07 & 3.83 & 3.83 & 3.48 & 3.48 & 3.71 & 3.06 & 3.58 \\
\hline Glucose Run 1 & 3.83 & 3.88 & 3.38 & 3.64 & 3.76 & 3.83 & 3.42 & 3.52 \\
\hline Glucose Run 1 & 4.15 & 4.05 & 3.46 & 3.69 & 3.57 & 3.99 & 3.2 & 3.59 \\
\hline Glucose Run 1 & 3.98 & 3.65 & 3.89 & 3.51 & 3.3 & 3.96 & 3.14 & 3.41 \\
\hline Glucose Run 1 & 3.95 & 3.72 & 3.21 & 3.72 & 3.52 & 3.91 & 3.09 & 3.64 \\
\hline Glucose Run 2 & 4.04 & 4.25 & 4.12 & 4.16 & 3.93 & 4.24 & 3.45 & 3.87 \\
\hline Glucose Run 2 & 4.15 & 4.65 & 3.83 & 3.91 & 4.04 & 4.31 & 3.41 & 3.86 \\
\hline Glucose Run 2 & 4.68 & 4.75 & 4.02 & 3.75 & 4.09 & 4.33 & 3.56 & 3.88 \\
\hline Glucose Run 2 & 4.3 & 4.84 & 3.86 & 3.77 & 4.24 & 4.21 & 3.33 & 3.9 \\
\hline Glucose Run 2 & 4.35 & 4.92 & 4.34 & 3.73 & 4.09 & 4.45 & 3.28 & \\
\hline avg & 4.03 & 4.05 & 3.69 & 3.73 & 3.73 & 4.06 & 3.37 & 3.58 \\
\hline std dev & 0.27 & 0.51 & 0.32 & 0.17 & 0.28 & 0.21 & 0.19 & 0.23 \\
\hline std dev/avg & $6.67 \%$ & $12.59 \%$ & $8.59 \%$ & $4.44 \%$ & $7.62 \%$ & $5.07 \%$ & $5.53 \%$ & $6.31 \%$ \\
\hline std err & 0.07 & 0.13 & 0.08 & 0.04 & 0.07 & 0.05 & 0.05 & 0.06 \\
\hline std err/avg & $1.72 \%$ & $3.25 \%$ & $2.22 \%$ & $1.15 \%$ & $1.97 \%$ & $1.31 \%$ & $1.43 \%$ & $1.69 \%$ \\
\hline
\end{tabular}

Table 8. ANOVA table for tryptophan diffusivity generated using JMP.

\begin{tabular}{|l|l|l|l|l|l|}
\hline Source & Nparm & DF & Sum of Squares & F Ratio & Prob $>$ F \\
\hline Alginate & 1 & 1 & $6.70939 \mathrm{e}-8$ & 0.3471 & 0.6611 \\
\hline X-Link Conc & 1 & 1 & $1.42429 \mathrm{e}-8$ & 0.0737 & 0.8313 \\
\hline X-Link Time & 1 & 1 & $4.6179 \mathrm{e}-8$ & 0.2389 & 0.7106 \\
\hline Alginate*X-Link Conc & 1 & 1 & $2.25579 \mathrm{e}-7$ & 1.1671 & 0.4754 \\
\hline Alginate*X-Link Time & 1 & 1 & $4.72279 \mathrm{e}-8$ & 0.2443 & 0.7077 \\
\hline X-Link Conc*X-Link Time & 1 & 1 & $8.30677 \mathrm{e}-9$ & 0.0430 & 0.8699 \\
\hline
\end{tabular}


Table 9. ANOVA table results for run 1 of glucose diffusivity generated using JMP.

\begin{tabular}{|l|l|l|l|l|l|}
\hline Source & Nparm & DF & Sum of Squares & F Ratio & Prob > F \\
\hline Alginate & 1 & 1 & $3.327 \mathrm{e}-8$ & 8.3674 & 0.2119 \\
\hline X-Link Conc & 1 & 1 & $1.18841 \mathrm{e}-8$ & 2.9889 & 0.3338 \\
\hline X-Link Time & 1 & 1 & $9.99624 \mathrm{e}-8$ & 25.1406 & 0.1253 \\
\hline Alginate*X-Link Conc & 1 & 1 & $2.60154 \mathrm{e}-7$ & 65.4288 & 0.0783 \\
\hline Alginate*X-Link Time & 1 & 1 & $2.20898 \mathrm{e}-8$ & 5.5556 & 0.2554 \\
\hline X-Link Conc*X-Link Time & 1 & 1 & $2.55247 \mathrm{e}-7$ & 64.1947 & 0.0790 \\
\hline
\end{tabular}

Table 10. ANOVA table results for run 2 of glucose diffusivity generated using JMP.

\begin{tabular}{|l|l|l|l|l|l|}
\hline Source & Nparm & DF & Sum of Squares & F Ratio & Prob > F \\
\hline Alginate & 1 & 1 & $4.67501 \mathrm{e}-9$ & 0.2398 & 0.7101 \\
\hline X-Link Conc & 1 & 1 & $3.51617 \mathrm{e}-8$ & 1.8034 & 0.4075 \\
\hline X-Link Time & 1 & 1 & $1.88208 \mathrm{e}-8$ & 0.9653 & 0.5056 \\
\hline Alginate*X-Link Conc & 1 & 1 & $6.21306 \mathrm{e}-9$ & 0.3187 & 0.6728 \\
\hline Alginate*X-Link Time & 1 & 1 & $2.07378 \mathrm{e}-9$ & 0.1064 & 0.7993 \\
\hline X-Link Conc*X-Link Time & 1 & 1 & $6.99857 \mathrm{e}-9$ & 0.3589 & 0.6564 \\
\hline
\end{tabular}

Table 11. ANOVA table results for combined glucose diffusivity runs generated using JMP.

\begin{tabular}{|l|l|l|l|l|l|}
\hline Source & Nparm & DF & Sum of Squares & F Ratio & Prob > F \\
\hline Alginate & 1 & 1 & $1.5722 \mathrm{e}-8$ & 10.7242 & 0.1887 \\
\hline X-Link Conc & 1 & 1 & $1.5406 \mathrm{e}-9$ & 1.0509 & 0.4921 \\
\hline X-Link Time & 1 & 1 & $5.13834 \mathrm{e}-8$ & 35.0493 & 0.1065 \\
\hline Alginate*X-Link Conc & 1 & 1 & $8.66939 \mathrm{e}-8$ & 59.1351 & 0.0823 \\
\hline Alginate*X-Link Time & 1 & 1 & $2.65673 \mathrm{e}-9$ & 1.8122 & 0.4067 \\
\hline X-Link Conc*X-Link Time & 1 & 1 & $4.44287 \mathrm{e}-8$ & 30.3054 & 0.1144 \\
\hline
\end{tabular}

Table 12. Fit report of the prediction model in JMP to tryptophan diffusivity responses.

\begin{tabular}{|l|l|}
\hline RSquare & 0.678883 \\
\hline RSquare Adj & -1.24782 \\
\hline Root Mean Square Error & 0.00044 \\
\hline Mean of Response & 0.00061 \\
\hline Observations (or Sum Wgts) & 8 \\
\hline
\end{tabular}


Table 13. Fit report of the prediction model in JMP to glucose diffusivity responses.

\begin{tabular}{|l|l|}
\hline RSquare & 0.994209 \\
\hline RSquare Adj & 0.959462 \\
\hline Root Mean Square Error & 0.000063 \\
\hline Mean of Response & 0.000693 \\
\hline Observations (or Sum Wgts) & 8 \\
\hline
\end{tabular}

Table 14. ANOVA table results for tryptophan initial concentration generated using JMP.

\begin{tabular}{|l|l|l|l|l|l|}
\hline Source & Nparm & DF & Sum of Squares & F Ratio & Prob $>$ F \\
\hline Alginate & 1 & 1 & 0.00025050 & 0.5977 & 0.5810 \\
\hline X-Link Conc & 1 & 1 & 0.00087291 & 2.0829 & 0.3858 \\
\hline X-Link Time & 1 & 1 & 0.00114282 & 2.7269 & 0.3466 \\
\hline Alginate*X-Link Conc & 1 & 1 & 0.00185467 & 4.4255 & 0.2825 \\
\hline Alginate*X-Link Time & 1 & 1 & 0.00146652 & 3.4993 & 0.3125 \\
\hline X-Link Conc*X-Link Time & 1 & 1 & 0.00088261 & 2.1060 & 0.3841 \\
\hline
\end{tabular}

Table 15. ANOVA table results for glucose initial concentration run 1 generated using JMP.

\begin{tabular}{|l|l|l|l|l|l|}
\hline Source & Nparm & DF & Sum of Squares & F Ratio & Prob > F \\
\hline Alginate & 1 & 1 & 52.44358 & 1.1713 & 0.4749 \\
\hline X-Link Conc & 1 & 1 & 0.38823 & 0.0087 & 0.9409 \\
\hline X-Link Time & 1 & 1 & 489.32139 & 10.9283 & 0.1870 \\
\hline Alginate*X-Link Conc & 1 & 1 & 3.06180 & 0.0684 & 0.8372 \\
\hline Alginate*X-Link Time & 1 & 1 & 17.72673 & 0.3959 & 0.6425 \\
\hline X-Link Conc*X-Link Time & 1 & 1 & 13.79264 & 0.3080 & 0.6774 \\
\hline
\end{tabular}

Table 16. ANOVA table results for glucose initial concentration run 2 generated using JMP.

\begin{tabular}{|l|l|l|l|l|l|}
\hline Source & Nparm & DF & Sum of Squares & F Ratio & Prob $>$ F \\
\hline Alginate & 1 & 1 & 44.00395 & 0.6099 & 0.5779 \\
\hline X-Link Conc & 1 & 1 & 10.15868 & 0.1408 & 0.7715 \\
\hline X-Link Time & 1 & 1 & 60.02495 & 0.8320 & 0.5292 \\
\hline Alginate*X-Link Conc & 1 & 1 & 9.03057 & 0.1252 & 0.7835 \\
\hline Alginate*X-Link Time & 1 & 1 & 6.97476 & 0.0967 & 0.8081 \\
\hline X-Link Conc*X-Link Time & 1 & 1 & 169.00109 & 2.3424 & 0.3684 \\
\hline
\end{tabular}


Table 17. ANOVA table results for combined glucose initial concentration runs generated using JMP.

\begin{tabular}{|l|l|l|l|l|l|}
\hline Source & Nparm & DF & Sum of Squares & F Ratio & Prob > F \\
\hline Alginate & 1 & 1 & 0.092491 & 0.1139 & 0.7928 \\
\hline X-Link Conc & 1 & 1 & 3.629692 & 4.4681 & 0.2813 \\
\hline X-Link Time & 1 & 1 & 51.646019 & 63.5749 & 0.0794 \\
\hline Alginate*X-Link Conc & 1 & 1 & 0.393938 & 0.4849 & 0.6128 \\
\hline Alginate*X-Link Time & 1 & 1 & 0.615703 & 0.7579 & 0.5440 \\
\hline X-Link Conc*X-Link Time & 1 & 1 & 69.838499 & 85.9694 & 0.0684 \\
\hline
\end{tabular}

Table 18. Fit report of the prediction model in JMP to tryptophan initial concentration responses.

\begin{tabular}{|l|r|}
\hline RSquare & 0.939167 \\
\hline RSquare Adj & 0.574169 \\
\hline Root Mean Square Error & 0.020472 \\
\hline Mean of Response & 0.149571 \\
\hline Observations (or Sum Wgts) & 8 \\
\hline
\end{tabular}

Table 19. Fit report of the prediction model in JMP to glucose initial concentration responses.

\begin{tabular}{|l|r|}
\hline RSquare & 0.927957 \\
\hline RSquare Adj & 0.495697 \\
\hline Root Mean Square Error & 6.691456 \\
\hline Mean of Response & 44.33706 \\
\hline Observations (or Sum Wgts) & 8 \\
\hline
\end{tabular}




\section{APPENDIX B}

\section{RECOMMENDED EXPERIMENTAL PROTOCOL}

\section{A. Purpose of this Guide}

This guide is intended to serve as recommended experimental protocol for the senior chemical engineering lab course at San Jose State University. Recommendations in this guide come from a survey of relevant literature and experimental results presented in this thesis.

\section{B. Objective of Experiment}

Use design of experiments to compare different polymerization conditions in alginate beads and calculate how those conditions affect the diffusivity of solutes through the alginate membrane.

C. Concepts Covered in this Lab

Polymerization, design of experiments, non-linear differential equations, diffusion, $\mathrm{UV} / \mathrm{Vis}$, statistical analysis

D. Recommendations for Solutes

\begin{tabular}{|l|l|}
\hline Solute & Quantitation method \\
\hline Glucose $[15,30]$ & $\begin{array}{l}\text { Colorimetric with Glucose Oxidase } \\
\text { Reagent, Absorbance at } 500 \mathrm{~nm}\end{array}$ \\
\hline Tryptophan [15] & Absorbance at $280 \mathrm{~nm}$ \\
\hline Immunoglobulin [6] & Absorbance at $280 \mathrm{~nm}$ \\
\hline Creatinine & Absorbance at $520 \mathrm{~nm}$ \\
\hline o-nitrophenol [22] & Absorbance at $420 \mathrm{~nm}$ \\
\hline Ethanol [17] & Gas chromatography \\
\hline
\end{tabular}




\section{E. Alginate Bead Generation}

Recommended range for levels:

Alginate concentration: $0.5-2.5 \% \mathrm{w} / \mathrm{v}$

Calcium chloride concentration: $0.1-15 \% \mathrm{w} / \mathrm{v}$

Cross-linking time: 5 minutes -2 hours

Note: Concentrations up to $4 \% \mathrm{w} / \mathrm{v}$ alginate have been used in literature [15], however would likely need over one hour to prepare alginate solutions, and may lead to clogging of syringes or uniformity issues.

Protocol:

1. Dissolve medium viscosity alginate in deionized water by stirring on a stir plate. Complete mixing may take over an hour. Alginate solutions may be prepared in advance and stored in the refrigerator.

2. Pour calcium chloride solution in a wide mouth container such as a large beaker or petri dish. A large surface area is desired to mitigate alginate beads joining together.

3. Load alginate solution into disposable syringe. Invert syringe and push out any large bubbles or air pockets using the plunger.

4. While stirring or swirling the container with the calcium chloride solution, drop the alginate solution by slowly pushing the syringe with smooth, even pressure. Observe immediate cross-linking of alginate beads. It is recommended to keep the distance between the syringe and the cross-linker solution constant to ensure uniformity [6]. Readjust the distance if beads are not spherical. 
5. Allow alginate beads to incubate in cross-linking solution for the cross-link time.

6. Carefully decant or drain cross-linking solution and dispose. Pour an excess amount of $0.9 \% \mathrm{w} / \mathrm{v}$ sodium chloride solution (saline wash) and decant or drain. Repeat the saline wash a total of 3 times.

7. Beads that float due to encapsulated bubbles should be removed and discarded.

F. Mass Transfer Experiments

Protocol:

1. Generate a calibration curve for the target solute using an appropriate quantitation method (see Recommendations for solutes).

2. Measure out the desired volume of beads by pouring in volumetric container. A volume less than 1 milliliter is not recommended, as it reduces reproducibility in the results.

3. Remove all fluid from beads using a pipette or blotting paper. Pour an excess volume of high concentration solution. The high concentration solution should be at least 10 times higher than the highest absorbance point on the calibration curve. This will account for the dilution of the solute as it diffuses out of the membrane and into the bulk fluid.

4. Incubate alginate beads in high concentration solution overnight.

5. Decant or drain high concentration solution and pour alginate beads in a clean dry beaker on a stir plate with the stirrer spinning.

6. At the same moment, start a timer and pour a solution free of solute over the beads. The volume should be 10 times the volume of alginate beads. 
7. Remove small volume samples at regular or pre-determined times. Plan the number of samples and their collective volume to total less than $10 \%$ volume removed from the bulk.

8. Measure the concentration of solute in each sample using the quantitation method appropriate for the target solute.

9. Repeat steps 2-9 for all other bead samples. Measure the bead diameter of a minimum of five randomly chosen beads at the end of each run using a micrometer or calipers.

G. Data Analysis

This experiment is intended to allow a framework for students to develop their own experimental matrix using DOE. Many factors can be varied and analyzed for their impact on the response. Once the mass transfer experiments are run and concentration versus time data is gathered for all conditions in the experimental matrix, the diffusivity can be determined.

Refer to Crank [18] for the development of Equation 3, which models diffusion of a solute from a sphere into bulk fluid initially free of solute. Use nonlinear regression tools, such as MATLAB, Mathematica, or PolyMath to solve for diffusivity for all runs in the experimental matrix.

Main factors and interactions can be found by following traditional DOE analysis. For examples on this approach, the NIST Handbook of Statistical Methods [9], Box [10], Verma [11], and Bass [27] are good resources. 